\title{
The Effect of
}

Two-Dimensional

Topography on Superficial Thermal Gradients

GEOLOGICAL SURVEY BULLETIN 1203-E 


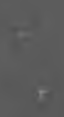

if

$y$

I)

$\because$

$$
\text { - }
$$




\section{The Effect of}

\section{Two-Dimensional}

\section{Topography on Superficial Thermal Gradients}

By ARTHUR H. LACHENBRUCH

EXPERIMENTAL AND THEORETICAL GEOPHYSICS

G E O L O I I A L S U R VEY B U L L E T I N 1203-E

Steady heat flux through an inclined plane of arbitrary height and slope angle and its application to geothermal measurements

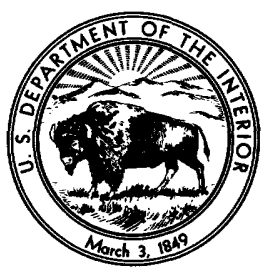


UNITED STATES DEPARTMENT OF THE INTERIOR

WALTER J. HIGKEL, Secretary

\author{
GEOLOGICAL SURVEY \\ William T. Pecora, Director
}




\section{CONTENTS}

Page

Abstract

Introduction $\ldots$

Acknowledgments . .

Heat flow through a plane slope: exact solution

Heat flow behind the brink and in front of the toe........ 8

Heat flow on the sloping surface

Heat flow through a plane slops: approximate solution

Comparison of the exact and approximate solutions._.

The problem of the general two-dimensional slope

Heat flux on a horizontal surface between two plane slopes_._._.

Case 1, plane valley

Case 2, plane ridge.

Case 3, plane bench... 32

Geometric bracketing . .

Equivalent slopes....

Tests of inequality $71 \ldots$

Slopes concave at the toe or convex at the brink 46

Some conditions for validity of the Jeffreys approximation...... 49

Bracketing with equivalent plane slopes: numerical results........ 50

Approximating with a single equivalent plane slope, $\bar{h}_{\mathrm{a}} \ldots \ldots \ldots \ldots$

Stations on gently sloping surfaces

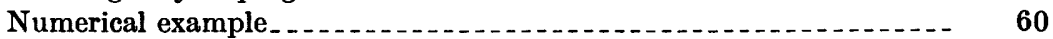

Variation of the topographic anomaly with depth

Case 1, all topography of one sign: general _...

$\begin{array}{ll}\text { Case 2, relief vanishes near the station and is of one sign elsewhere } & 67\end{array}$

Case 3, relief near the station is of one sign and it vanishes elsewhere. - $\quad 69$

Case 4, topography of both signs: general _... 70

Case 5, variation of the flattening error with depth 71

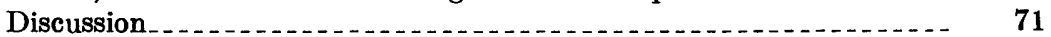

Ocean-bottom gradient measurements... 73

Time dependence and other effects... 75

Buried bedrock slope..... 77

Summary

References cited.......... 85

\section{ILLUSTRATIONS}

Plate 1. Graphs of heat flow through horizontal surfaces beyond the toe and behind the brink of a plane slope..... In pocket

2. Graph of Jeffreys approximation as a function of distance from the toe or brink of a plane slope.......... In pocket 
Figure

1. Diagram illustrating the transformation of the plane boundary of a half space into a plane slope.........

2-6. Graphs:

2. Vertical component of normalized heat flow through a plane slope.........................

3. Decay of the heat-flow anomaly with distance beyond the toe and behind the brink .........

4. Vertical component of heat flow through the sloping part of a plane slope

5. Difference between the exact solution and the Jeffreys approximation beyond the toe and behind the brink of a plane slope

Page

E5

6. Difference between the exact solution and the Jeffreys approximation on the inclined part of a plane slope

7. Diagrammatic comparison of the reference-plane temperatures required by the exact solution and Jeffreys approximation

8-13. Diagrams:

8. Coexisting plane slopes forming a plane valley, a plane ridge, and a plane bench

9. Geometrical significance of equation $29 . \ldots \ldots$.

10. Plane slope with a positive or negative bump...

11. Geometric bracketing of a general slope by two

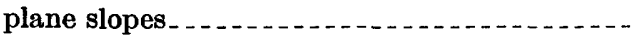

12. Notation for the discussion of slope forms .....

13. Notation for the discussion of equivalent plane

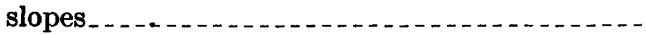

14. Graph comparing the anomaly caused by a plane slope with the anomaly from bracketing equivalent plane slopes.

15. Graph of error in the Jeffreys approximation as a function of the Jeffreys approximation for constant positive slope angle

16. Graph of error in the Jeffreys approximation as a function of the Jeffreys approximation for constant negative slope angle

17-20. Diagrams:

17. Numerical examples of equivalent slopes...... -

18. Flattening procedure . . .

19. Numerical example 1, stations on gently sloping surfaces

20. Numerical example 2, stations on gently sloping surfaces.

21. Graph of factors for depth dependence of the integrand in expressions for the gradient anomaly ...........

22. Diagram of general two-dimensional relief and the notation for equation 108

23. Diagram of model of the downfaulted bedrock pediment_ 


\section{TABLES}

1. Normalized surface heat flow $q$ at distance $r$ beyond toe of plane slope with angle beta and unit height........

2. Normalized surface heat flow $q$ at distance $s$ behind brink of plane slope with angle beta and unit height.......

3. Vertical component of surface heat flow $q$ at horizontal distance $w$ from brink of plane slope with angle beta and unit width

4. Comparison of exact solutions for families of positive equivalent plane slopes..............................

5. Comparison of exact solutions for families of negative

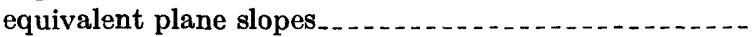

6. Time for approach of surface heat flow to equilibrium after generation of a cliff at distance $x$.............. 



\title{
EXPERIMENTAL AND THEORETICAL GEOPHYSICS
}

\section{THE EFFEGT OF TWO-DIMENSIONAL TOPOGRAPHY ON SUPERFIGIAL THERMAL GRADIENTS}

\author{
By Arthur H. Lachenbruch
}

\begin{abstract}
The Jeffreys-Bullard theory of the topographic disturbance to geothermal gradients is only approximate because it neglects the effects of lateral heat loss through sloping surfaces. It cannot be applied with confidence where the height of the relief features is large relative to their horizontal distance from the station and to the depth of the measurement points. Such conditions arise in the nearsurface geothermal measurements made in the ocean bottom if bold relief occurs on a scale exceeding a few meters, or on the continents in observations in shallow boreholes in rugged terrain.

In an important special case, the measurement depth is small relative to the distance to the relief, and the gradient anomaly can be approximated by the value applicable at zero depth. To investigate this case, an exact solution was obtained for the steady heat flux through an inclined plane of arbitrary height and slope angle. These two parameters are easily visualized and represented graphically so that models which approximate or bracket real topography can often be identified quickly. The effects of slopes of fairly general (two-dimensional) form can be approximated by identifying them with their equivalent plane slopes-the plane slopes which yield the same Jeffreys approximation at the station. The results can be applied to stations on planes, valleys, ridges, or benches bounded by irregular slopes. They are valid at points arbitrarily close to slopes of any height or inclination. Finite slope and curvature of the surface at the station can be accommodated if they are not too great. Although direct application of the theory is limited to cases in which the measurement depth is less than the horizontal distance to the relief, useful limits can be obtained for other cases. Even where other theories of the topographic correction are applicable, the present method can be useful as it leads to rapid estimates by graphical means.

In oceanic heat-flow measurements, the uncertainty in the topographic anomaly is less than 10 percent only if the probe length is at least 2 or 3 times the uncertainty in local elevation differences, and the curvature of the temperature profile is negligible. Over an irregular ocean bottom, relief not detectable by modern echo-sounding techniques could cause errors of 50-100 percent in gradient measurements to depths of a few meters. Such errors will often, but not always, be accompanied by marked curvature in the temperature profile. Heat-flow anomalies of several hundred percent, such as some reported from oceanic ridges, cannot be attributed to undetected relief. The steady-state topographic anomaly in the center of a deep narrow oceanic trench can be on the order of 25 percent.
\end{abstract}


The theory may be applied also to topographic corrections in lakes and boreholes on the continents, and to the case of thermal refraction across a sloping bedrock surface buried in alluvium.

\section{INTRODUCTION}

Topographic relief causes local irregularities in near-surface geothermal gradients and they must be identified before regionally meaningful values of geothermal flux can be obtained. A steady-state theory of the topographic correction was presented by Jeffreys (1938) and elaborated by Bullard (1938). A comprehensive discussion by Birch (1950) extended the theory to account for topographic evolution. The fundamental simplification in these corrections results from replacing the irregular surface by a reference plane upon which the temperature varies in proportion to the relative elevation of the actual surface. There is no limit to the fidelity with which the topographic surface can be represented, as the representation is achieved by a numerical procedure. The mathematical approximation of Jeffreys was verified by Birch (1950) for subsurface points by comparison with exact solutions for simple topographic models obtained by superimposing a uniform field and the fields of continuous point or line doublets (Lees, 1910).

In an alternative approach, Jaeger and Sass (1963) approximated the topography with Lees' line-doublet model and calculated the subsurface temperature corrections directly (Jaeger, 1965, p. 10). The Lees' model gives results for symmetrical ridges or valleys (depending on the polarity of the doublet) with geometric parameters determined by the distance of the doublet above the surface and the relative strengths of the doublet and uniform field. Integrating the doublet solution over a half plane, Jaeger and Sass obtained the solution for a monocline, that is, a family of monotone sloping surfaces asymptotic to horizontal planes at different elevations. Other exact solutions for special topographic forms have been given by Castoldi (1952) and in various works discussed by Birch (1950).

Thus, in the two classical approaches to the geothermal terrain correction, one approach considers the approximate effects of an (effectively) exact representation of topography, and the other, the exact effects of an approximate representation of topography. As Birch (1950) has shown, the first method is more general, and it lends itself readily to refinements accounting for topographic evolution. It has been pointed out by Jaeger and Sass (1963) that the second method is useful for very rapid estimates of terrain effects where detailed corrections are not warranted because of imperfectly known topography or other uncertain sources of disturbance. This last statement depends upon the geometric model being sufficiently 
simple that it can be easily identified with the topographic surface to be approximated. Both methods generally become less satisfactory as the gradient measurement to be corrected approaches the surface. This can be explained as follows: In general the frequency of occurrence of features of the earth's topographic relief decreases as the size of the feature increases; the largest and most infrequent features have a vertical scale on the order of a few kilometers. However, the effect of topographic features on the geothermal gradient is not large as long as their height is (1) less than the horizontal distance to the measurement point, or (2) less than the depth of measurement. Thus, for measurements a few kilometers beneath the surface, condition 2 is satisfied even for the most rugged relief (at any horizontal distance), and only a gross representation of the topography, used with any reasonable approximation scheme will generally suffice. As the measurement points approach the surface, progressively smaller (and more frequently occurring) features will fail to satisfy condition 2, and those that are not far enough from the station to satisfy condition 1 will have to be accounted for with progressively increasing rigor; although the effects of distant relief do not diminish, the effects of close-in relief increase greatly, and small-scale irregularities can cause sizable anomalies. Under these conditions the second method becomes less satisfactory because it becomes increasingly difficult to represent close-in relief in detail and still account for distant relief in a gross way with a simple geometric model. The first method becomes uncertain for near-surface measurements because it neglects secondorder effects of lateral variations of the vertical gradient in the relief. These effects can become appreciable when close-in relief must be considered. Birch (1950, p. 625) pointed out that, "at shallow depths, under sharp irregularities, the approximation is sure to be poor." For this report it is necessary to define "shallow" or "near-surface" gradient measurements. For this purpose the term "superficial gradient" will be used to refer to a gradient in which the topographic anomaly is approximated well by the value applicable at the surface $(z=0)$.

Almost 90 percent of the determinations to date of earth heat flow were computed from measurements of gradient made within a few meters of the surface of the solid earth (Lee and Uyeda, 1965). They were made beneath the world's oceans and seas where effects of topographic relief are surely unimportant over much, but not all, of the bottom. Generally these measurements are not corrected for topographic effects because continuous precision depth soundings and bottom photographs indicate that there is no nearby relief with a scale exceeding a few meters, because the bottom topography is not adequately known, or because there is no readily available simple 
method of estimating the effects. In most determinations (Bullard and others, 1956) it will be found that the bottom topography is not known well enough to permit detailed corrections. Investigation of the problem is necessary to ascertain what must be known of the topography near a superficial gradient measurement in order that it might be reduced to a regionally meaningful quantity. This problem is considered for the two-dimensional case in the present paper. In return for the loss of generality imposed by the two-dimensional model, we gain an intuitive simplicity that makes the results easy to visualize and apply. The effects of many real topographic surfaces can be approximated or bracketed by the two-dimensional model.

An additional limitation of the present treatment is that most of the results apply only to the limiting value of the topographic anomaly as the depth approaches zero; that is, they apply to superficial gradient measurements. Although the superficial case includes many important trouble spots for the Jeffreys approximation, it does not include them all.

The plan of this paper is as follows:

Exact expressions are derived for the anomaly in the vertical surface flux caused by a plane slope, defined as an inclined plane segment joining two horizontal halfplanes of different elevation.

The corresponding analytical result for the plane slope that follows from Jeffreys' reference-plane assumption is given.

The two results are compared.

Upper and lower limits are given for the heat flow where two plane slopes coexist to form a plane valley, plane ridge, or plane bench.

A simple procedure is described for bracketing the anomaly due to a general slope by exact results for plane slopes.

The first and second methods for terrain corrections (described previously in introduction) are combined to obtain a method for approximating and bracketing the effects of a general slope, Conditions for neglecting gentle slopes at the station are derived.

Variation of the topographic anomaly with depth is discussed, and conditions under which a gradient anomaly may be considered superficial are presented.

Transient effects are considered and an additional application, involving the heat-flow anomaly caused by a buried bedrock pediment, is given for previously obtained results.

\section{ACKNOWLEDGMENTS}

I am grateful to Dr. J. H. Sass and Dr. W. H. K. Lee for reviewing this manuscript and providing many useful suggestions, and to Dr. S. H. Lachenbruch for checking much of the mathematical material and calling my attention to inconsistencies in notation. Informal 
comments on the manuscript were offered by Professor Francis Birch, Dr. John Reitzel, Dr. Richard Von Herzen, Professor William Diment, and Dr. Allan Cox. They were helpful and are gratefully acknowledged. Roger Bowen and John Tanida provided invaluable assistance with numerical computation and programming.

This work is part of a continuing geothermal study by the U.S. Geological Survey. It is supported in part by the Office of Naval Research (Task NR 307-107 and NR 307-275).

\section{HEAT FLOW THROUGH A PLANE SLOPE: EXACT SOLUTION}

An expression is needed for the vertical thermal flux through a plane slope on the earth's surface. The earth is assumed to be homogeneous and isotropic, and the surface is represented by a plane segment inclined at an angle $\beta$ to horizontal surfaces beyond the toe and behind the brink which are at an elevation difference $H$. The model is illustrated by the region below the contour $\Gamma^{\prime}\left(A^{\prime}, B^{\prime}, C^{\prime}, D^{\prime}, E^{\prime}\right)$ in the $\eta$-plane, figure 1 . The slope angle $\beta$ is represented by $\pi / n$. To represent otherwise uniform conditions, it is assumed that the surface $\left(\Gamma^{\prime}\right)$ is at zero temperature, and that at large distances from the slope the temperature is proportional to distance beneath the surface. Therefore, in the $\eta$-plane we want to find

Subject to

$$
\left.\frac{\partial \theta}{\partial z}\right|_{\Gamma^{\prime}}
$$

$$
\begin{gathered}
\frac{\partial^{2} \Theta}{\partial x^{2}}+\frac{\partial^{2} \theta}{\partial z^{2}}=0 \\
\Theta=0,(x, z) \text { on } \Gamma^{\prime}
\end{gathered}
$$

$\eta$-plane

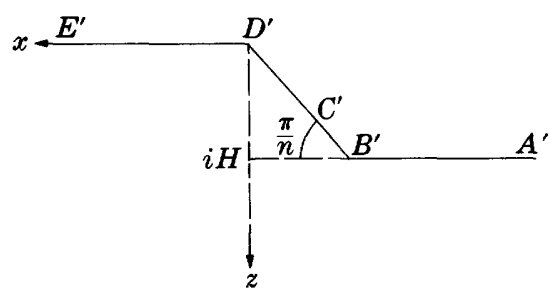

$\omega$-plane

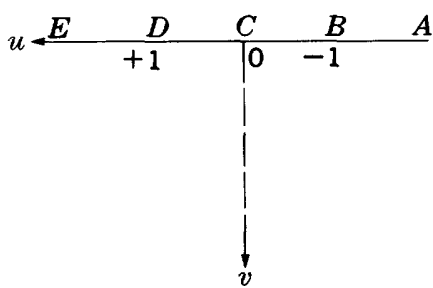

Figure 1.-Transformation of the plane boundary of a half space into a plane slope. 


$$
\frac{\partial \theta}{\partial z} \rightarrow G, \text { a constant }|x|, z \rightarrow \infty
$$

The special case of the vertical cliff $(n=2)$ has been discussed by Castoldi (1952). His solution was obtained by mapping a uniform field in the $\omega$-plane into the region bounded by a step-shaped contour of the $\eta$-plane with the conformal transformation

$$
\frac{d \eta}{d \omega}=A\left(\frac{\omega+1}{\omega-1}\right)^{1 / 2}
$$

The more general function (Kober, 1962, p. 161),

$$
\frac{d \eta}{d \omega}=A\left(\frac{\omega+1}{\omega-1}\right)^{\frac{1}{n}} \text { where } n \text { is a positive integer, }
$$

achieves the mapping illustrated in figure 1 . It follows, from the properties of conformal transformations, that this function maps the uniform field

$$
\theta^{*}(\omega)=\gamma v, \gamma \text { a constant, } v \geq 0,
$$

into the function $\theta(\eta)$ which satisfies conditions 1 and 2. It also satisfies condition 3 if

$$
\gamma=G A \text {. }
$$

Inasmuch as the ratio of normal derivatives on corresponding contours is the reciprocal of the magnitude of the derivative of the transformation, we have from 4 and 5

$$
\begin{aligned}
{\left[\frac{\partial \theta}{\partial n}\right]_{\Gamma^{\prime}} } & =\gamma\left[A\left|\left(\frac{\omega+1}{\omega-1}\right)^{\frac{1}{n}}\right|\right]_{\Gamma}^{-1} \\
& =G\left|\left(\frac{u+1}{u-1}\right)^{\frac{1}{n}}\right|^{-1} .
\end{aligned}
$$

We shall adopt the notation

$$
\begin{gathered}
\frac{u+1}{u-1}=\lambda(u) \\
\left|\lambda^{\frac{1}{n}}\right|=p(u, n) .
\end{gathered}
$$


Hence

$$
\begin{aligned}
\left.\frac{1}{G} \frac{\partial \theta}{\partial z}\right|_{\Gamma^{\prime}} & =\frac{1}{p},|u|>1 \\
& =\frac{\cos \frac{\pi}{n}}{p},|u|<1 .
\end{aligned}
$$

The expression on the left is the quantity sought. It is the ratio of the vertical heat flow through any part of the surface $\Gamma^{\prime}$ to the regional heat flow. It is expressed in terms of $p$ which is known in terms of $u$, and we should like to have it in terms of $x=R(\eta)$. To get $x$ as a function of $u$ we integrate equation 4 on the boundary $\Gamma$. Thus:

$$
\eta(u)=2 n A \int_{\lambda^{n}}^{\infty} \frac{t^{n}}{\left(t^{n}-1\right)^{2}} d t=A\left[\frac{2 \lambda^{\frac{1}{n}}}{\lambda-1}+2 \int_{\lambda^{n}}^{\infty} \frac{d t}{t^{n}-1}\right],
$$

which yields for $n$ an even integer (Gröbner and Hofreiter, 1949, table 16 , formula $12 \mathrm{~b}$ ):

$$
\left.\begin{array}{rl}
\eta(u)= & \frac{H}{\pi}\left\{\frac{n \lambda^{\frac{1}{n}}}{\lambda-1}+\ln \left[\frac{\lambda^{\frac{1}{n}}+1}{\lambda^{\frac{1}{n}}-1}\right]\right. \\
& +\sum_{\nu=1}^{\frac{n}{4}+\frac{\epsilon}{2}-1}\left[\cos \frac{2 \nu \pi}{n} \ln \left(\frac{\lambda^{\frac{2}{n}}+2 \lambda^{\frac{1}{n}} \cos \frac{2 \nu \pi}{n}+1}{\lambda^{\frac{2}{n}}-2 \lambda^{\frac{1}{n}} \cos \frac{2 \nu \pi}{n}+1}\right)\right. \\
& \left.-2 \sin \frac{2 \nu \pi}{n} \tan -1\left[\frac{1-\lambda^{\frac{2}{n}}}{2 \lambda^{\frac{1}{n}} \sin \frac{2 \nu \pi}{n}}\right)\right]+\frac{\pi}{2}\left[1-\epsilon-\sum_{\nu=1}^{\frac{n}{2}-1} \sin \frac{2 \nu \pi}{n}\right] \\
-2(1-\epsilon) \tan { }^{-1}\left(\lambda^{\frac{-1}{n}}\right)
\end{array}\right\},
$$

where

and $\lambda$ is defined by equation 7 .

$$
\begin{aligned}
& \epsilon=0, n / 2 \text { even, } \\
& =1, n / 2 \text { odd, }
\end{aligned}
$$

This result includes the value of the constant in equation 10

$$
A=\frac{n H}{2 \pi},
$$


which is obtained from the condition:

$$
n=H\left(i-\cot \frac{\pi}{n}\right) \text { when } u=-1 \text {, i.e., when } \lambda=0 .
$$

Equation 11 maps the boundary $\Gamma$ into $\Gamma^{\prime}$.

\section{HEAT FLOW BEHIND THE BRINK AND IN FRONT OF THE TOE}

The horizontal parts of $\Gamma^{\prime}$ to the left and right of the sloping part are represented respectively by $u<-1$ and $u>+1$. Over both these ranges $\lambda$ is positive and hence $\lambda^{\frac{1}{n}}$ is real and equal to $p$. The only imaginary quantity that appears in 11 for $|u|>1$ is the $i H$ generated by the second term for $u<-1$ as required by the mapping.

Thus the $x$-coordinate of a point on the horizontal portion of the surface $\Gamma^{\prime}$ at which the normalized heat flow is $\frac{1}{p}$ is given by:

$$
\begin{gathered}
x(p)=\frac{H}{\pi}\left\{\frac{n p}{p^{n}-1}+\ln \left|\frac{p+1}{p-1}\right|\right. \\
+\sum_{\nu=1}^{\frac{n}{4}+\frac{\epsilon}{2}-1}\left[\cos \frac{2 \nu \pi}{n} \ln \left(\frac{p^{2}+2 p \cos \frac{2 \nu \pi}{n}+1}{p^{2}-2 p \cos \frac{2 \nu \pi}{n}+1}\right)-2 \sin \frac{2 \nu \pi}{n} \tan ^{-1}\left(\frac{1-p^{2}}{2 p \sin \frac{2 \nu \pi}{n}}\right)\right] \\
\left.+\frac{\pi}{2}\left[1-\epsilon-\sum_{\nu=1}^{\frac{n}{2}-1} \sin \frac{2 \nu \pi}{n}\right]-2(1-\epsilon) \tan ^{-1} \frac{1}{p}\right\},|u|>1, \quad(12)
\end{gathered}
$$

where

$$
\begin{aligned}
\epsilon & =0, \frac{n}{2} \text { even } \\
& =1, \frac{n}{2} \text { odd. }
\end{aligned}
$$

Equation 12 is valid only for $n=$ even integer. The corresponding general expression for $n$ odd is not needed, as only the case $n=3$ (that is $\beta=60^{\circ}$ ) gives useful additional information. The result corresponding to equation 12, but valid for $n=3$, is (Gröbner and Hofreiter, 1949, table 16, formula 12b) 


$$
\begin{aligned}
x(p)=\frac{H}{\pi}\left\{\frac{3 p}{p^{3}-1}+\ln \left|\frac{\left(p^{2}+p+1\right)^{\frac{1}{2}}}{p-1}\right|\right. \\
\left.+\sqrt{3}\left[\tan ^{-1} \frac{2 p+1}{\sqrt{3}}-\frac{\pi}{2}\right]\right\},|u|>1, n=3 .
\end{aligned}
$$

In equations 12 and 13 the normalized surface heat flow, $p^{-1}$, ranges between 0 and $\infty$ with extremes occurring at the brink and toe of the slope respectively. Values less than unity occur behind the brink and greater than unity in front of the toe as might be expected.

\section{HEAT FLOW ON THE SLOPING SURFACE}

The part of $\Gamma$ defined by $|u|<1$ maps into the sloping part of $\Gamma^{\prime}$. In this region

$$
\lambda \equiv \frac{u+1}{u-1}<0,
$$

and $\lambda^{\frac{1}{n}}$ is not real in general and hence is not equal to $p$. To reduce 11 to an expression corresponding to 12 but valid on the sloping surface, we set

and hence

$$
\lambda=p^{n} e^{i \pi}
$$

$$
\lambda^{\frac{1}{n}}=p e^{\frac{i \pi}{n}(1+2 k)}, \pm k=0,1,2,3, * * * n-1 .
$$

As $k=-1$ satisfies the conditions of the transformation, we use

$$
\lambda^{\frac{1}{n}}=p e^{\frac{-i \pi}{n}},
$$

in equation 11 and extract the real part to obtain the relation between $p$ and $x$ on the slope.

$$
\begin{aligned}
x(p)= & \frac{H}{\pi}\left\{\frac{-n p}{p^{n}+1} \cos \frac{\pi}{n}+\frac{1}{2} \ln \frac{\left(p^{2}-1\right)^{2}+4 p^{2}\left(\sin \frac{\pi}{n}\right)^{2}}{\left(p^{2}+1-2 p \cos \frac{\pi}{n}\right)^{2}}\right. \\
+ & \frac{1}{2} \sum_{\nu=1}^{\frac{n}{4}+\frac{\epsilon}{2}-1} \cos \frac{2 \nu \pi}{n} \ln \\
& {\left[p^{4}+1+2 p^{2} \cos \frac{2 \pi}{n}-4 p^{2}\left(\cos \frac{2 \nu \pi}{n}\right)^{2}\right]^{2}+\left[4 \sin \frac{\pi}{n} \cos \frac{2 \nu \pi}{n} p\left(p^{2}-1\right)\right]^{2} } \\
& {\left[p^{4}+1+2 p^{2} \cos \frac{2 \pi}{n}+4 p^{2}\left(\cos \frac{2 \nu \pi}{n}\right)^{2}-4 \cos \frac{\pi}{n} \cos \frac{2 \nu \pi}{n} p\left(p^{2}+1\right)\right]^{2} }
\end{aligned}
$$




$$
\begin{aligned}
& -\sum_{\nu=1}^{\frac{n}{4}+\frac{\epsilon}{2}-1} \sin \frac{2 \nu \pi}{n} \tan ^{-1} \frac{4 \cos \frac{\pi}{n} \sin \frac{2 \nu \pi}{n} p\left(p^{2}-1\right)}{p^{4}+1-2 p^{2} \cos \frac{2 \pi}{n}-4 p^{2}\left(\sin \frac{2 \nu \pi}{n}\right)^{2}}+\delta \frac{\pi}{2} \\
& \left.-\frac{\pi}{2} \sum_{\nu=1}^{\frac{n-2}{2}} \sin \frac{2 \nu \pi}{n}+(1-\epsilon) \tan ^{-1} \frac{p^{2}-1}{2 p \cos \frac{\pi}{n}}\right\},|u|<1, n \text { even. }
\end{aligned}
$$

where:

and

$$
\begin{aligned}
\delta & =-1, p<p_{1} \\
& =0, p_{1}<p<p_{2} \\
. & =+1, p_{2}<p
\end{aligned}
$$

$$
\begin{aligned}
& p_{1}^{2}, p_{2}^{2} \text { are roots of } p^{4}+1-2 p^{2} \cos \frac{2 \pi}{n} 4 p^{2}\left(\sin \frac{2 \nu \pi}{n}\right)^{2}=0 . \\
& p_{1}^{2}<p_{2}^{2} .
\end{aligned}
$$

Numerical results from equations 12, 13, and 14 are presented in terms of the following notation, which is more convenient for the discussion:

$$
G=Q / K, \quad \text { where } Q \text { is the regional heat flux, }
$$
and $K$ is the thermal conductivity.

$\left.\begin{array}{l}p^{-1},|u|>1 \\ p^{-1} \cos \beta,|u|<1\end{array}\right\}=\frac{K}{Q} \frac{\partial \theta}{\partial z}=q$, normalized vertical heat flux at surface $\left(\Gamma^{\prime}\right)$.

$$
\stackrel{x}{\bar{H}}=s,
$$

distance behind brink (that is, to the left from $D^{\prime}$, fig. 1) in units of slope height.

$$
\frac{1}{H}[-x-H \cot \beta]=r,
$$

distance beyond toe (that is, to the right from $B^{\prime}$, fig. 1) in units of slope height.

$$
\frac{-x}{H \cot \beta}=w
$$

horizontal distance from brink (that is, to the right from $D^{\prime}$, fig. 1) in units of slope width.

$s, r$, and $w$ will always be used as positive quantities, and $w$ will never exceed 1. Thus stations on the lower horizontal half plane will be designated by a value of the coordinate $r$, those on the upper half plane will be designated by a value of $s$, and those on the slope by a value of $w$. When a station lies on the lower half plane, that is, beyond the toe, the relief will be referred to as positive, and when it lies on the upper half plane (behind the brink), the relief will be called negative. Where it is not convenient to indicate the sign of the relief 
by explicit reference to the coordinates $r$ and $s$, slopes below the station will be designated by a negative value of $\beta$ and those above it by a positive value of $\beta$.

The general form of the normalized surface heat flow $q(x)$ is best seen from figure 2, where the abscissa is in units of slope width.

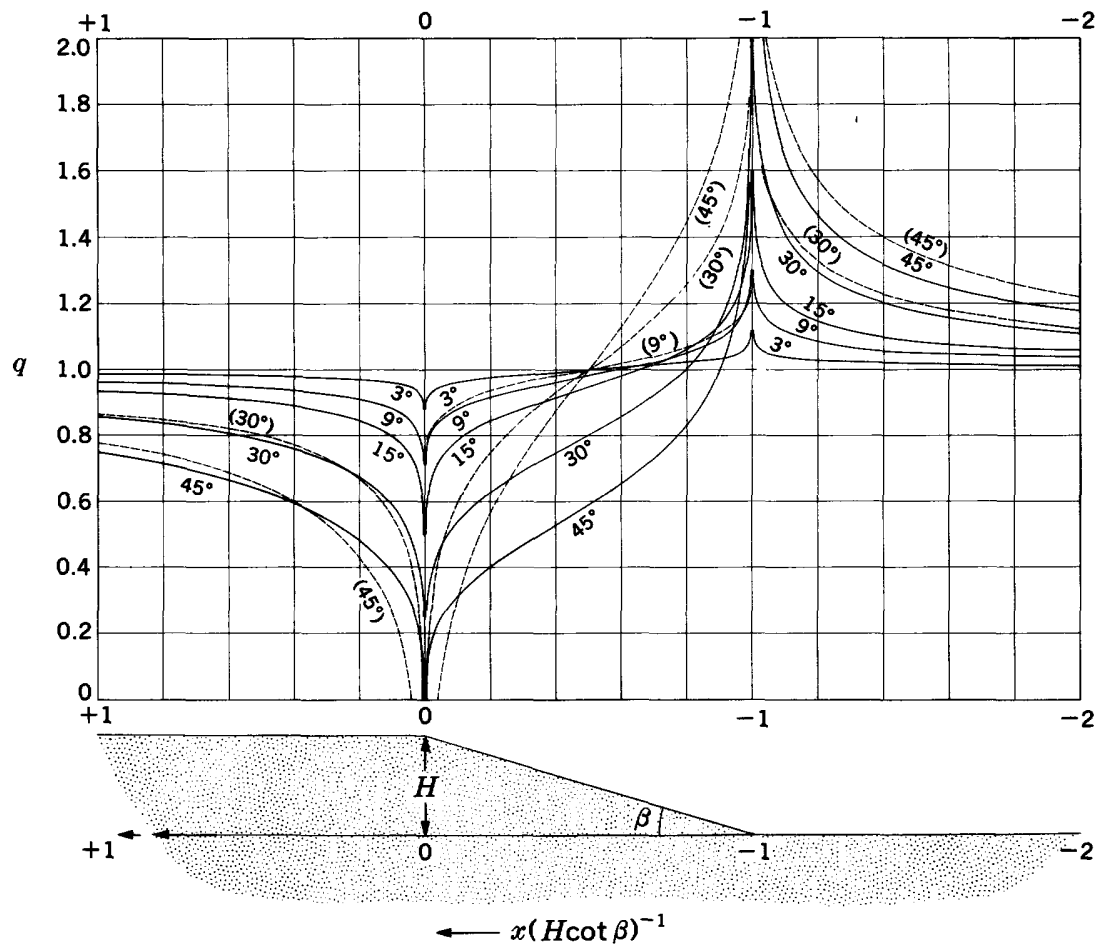

FIgURe 2.-Vertical component of normalized heat flow through a plane slope. Solid curves represent exact results, dashed curves represent the Jeffreys approximation.

However, when dealing with effects beyond the toe or behind the brink, it is usually more convenient to consider distances in units of slope height. This is done in figure 3 , which shows the decay of the topographic anomaly as a function of $r$ and $s$ for selected slope angles. The results 12,13 , and 14 are easily extended to other slope angles with graphs using $\beta$ as the ordinate (fig. 4 and pl. 1). Tabular results from equations 12 and 13 are presented in tables 1 and 2, and results from equation 14 are given in table 3 (p. E12-E14). 


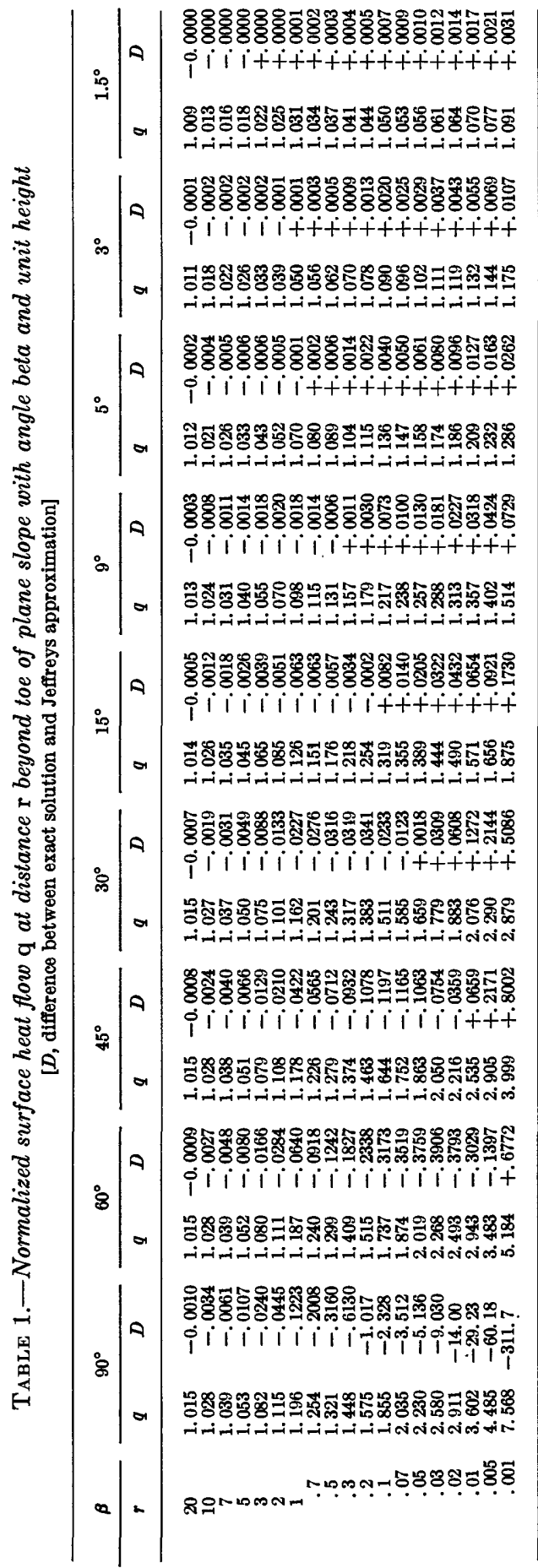




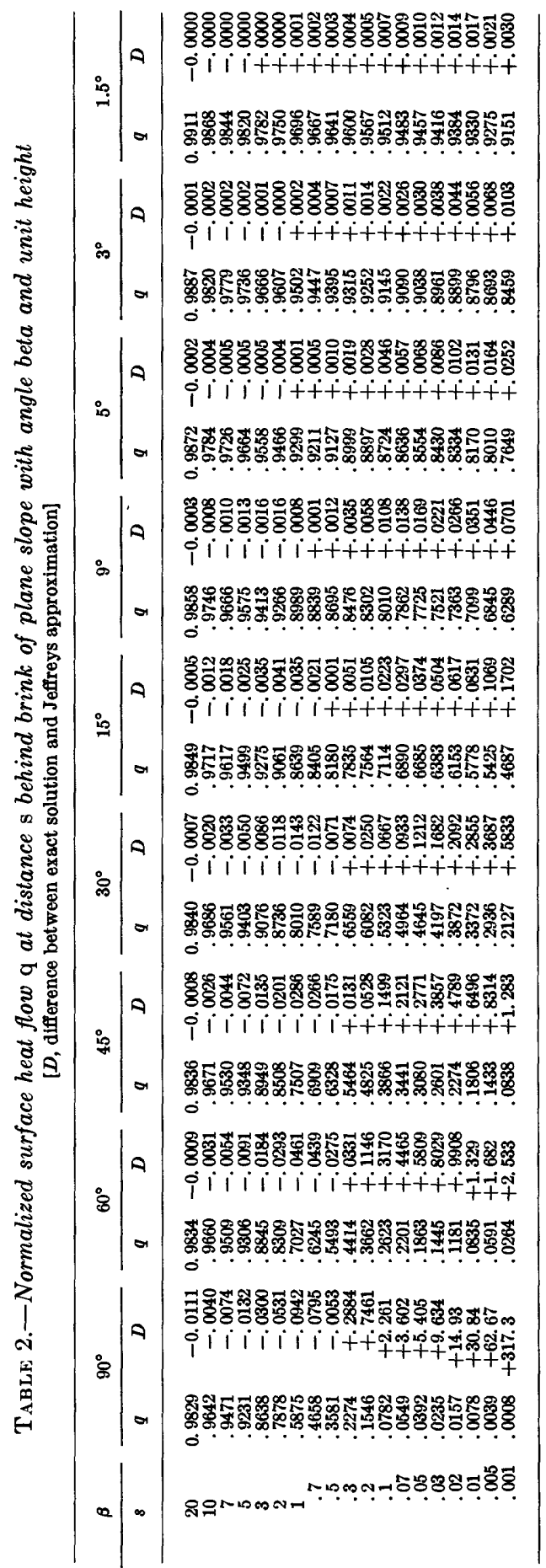




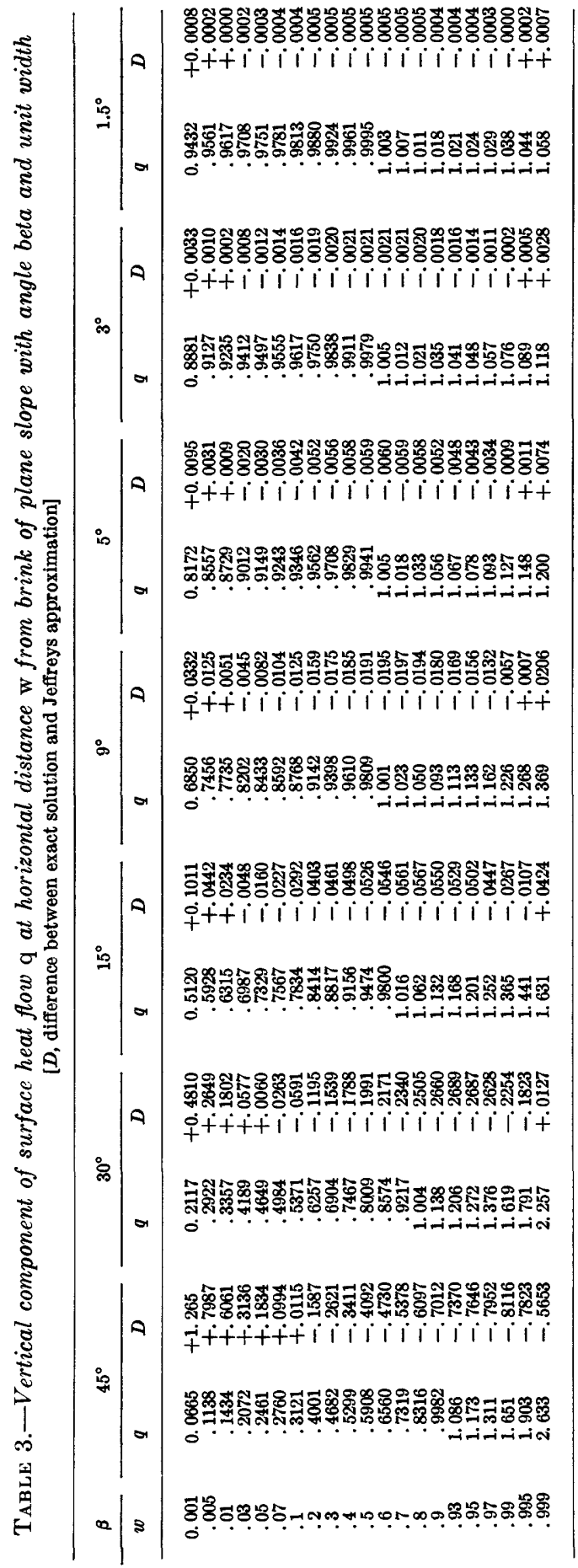


TOPOGRAPHY AND SUPERFICIAL THERMAL GRADIENTS E15

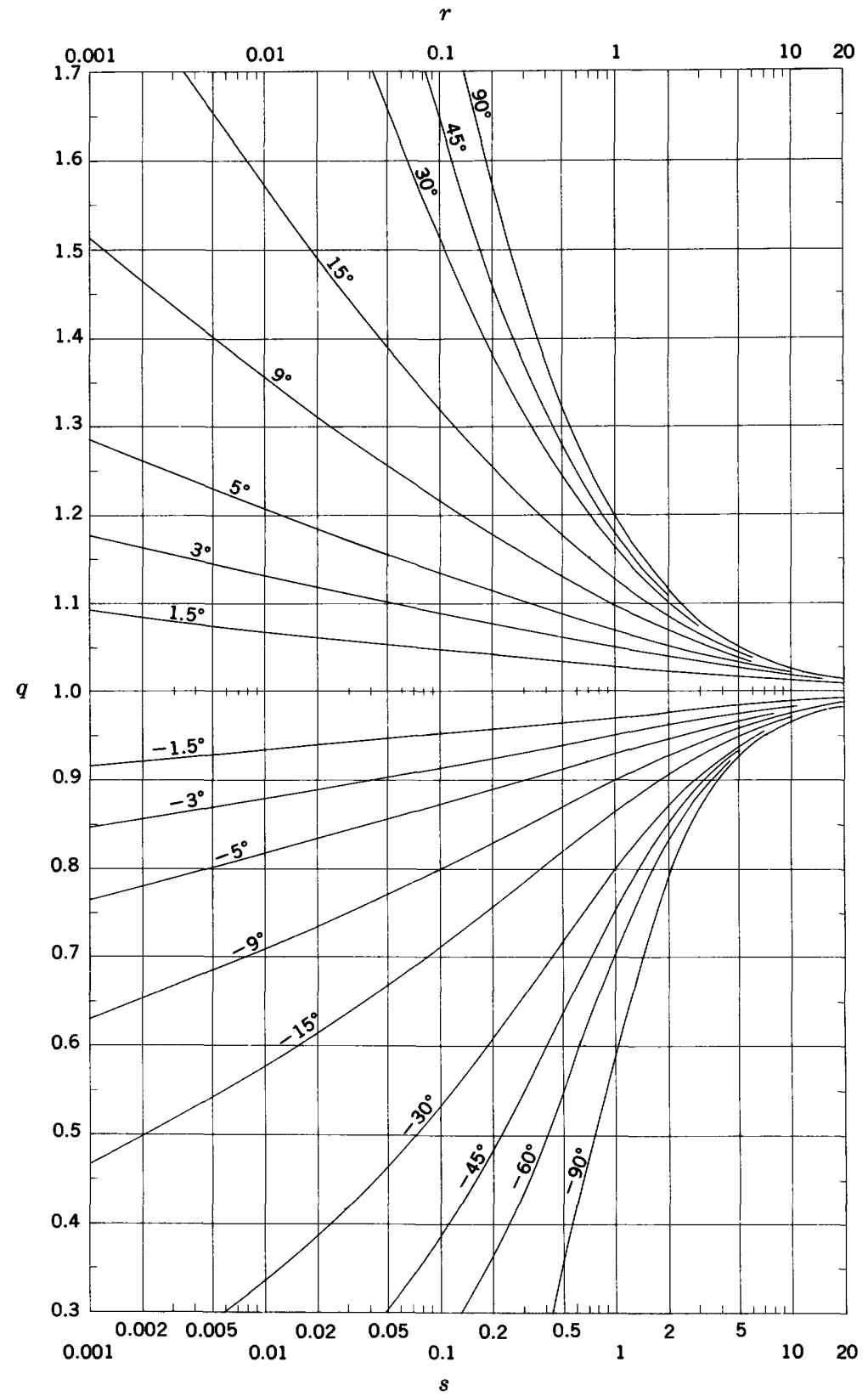

Figure 3.-Decay of the heat-flow anomaly with distance beyond the toe $(r$, upper curves) and behind the brink $(s$, lower curves) for selected slope angles $(\beta)$. 


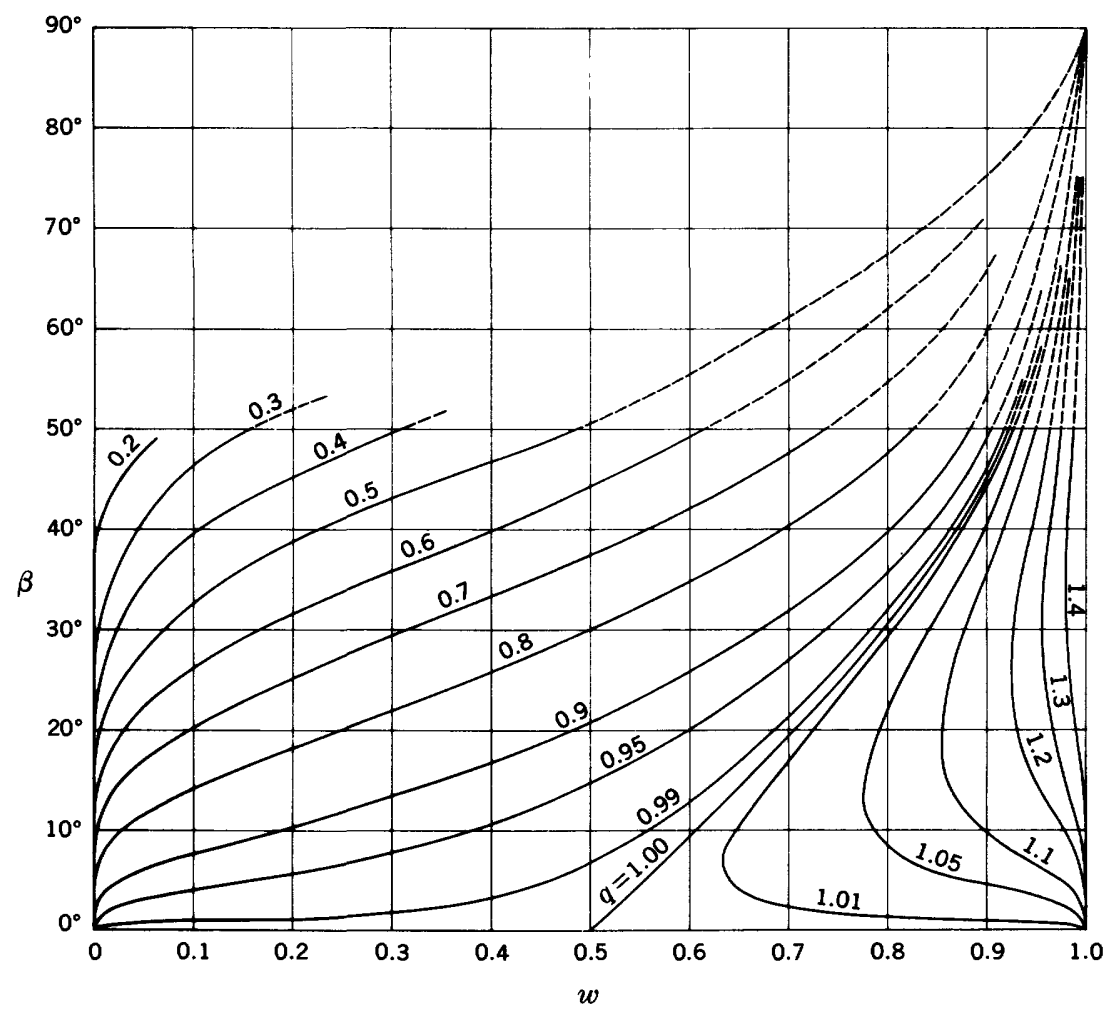

Figure 4.-Vertical component of heat flow through the sloping part of a plane slope. $w$ is horizontal distance from the brink in units of slope width.

\section{HEAT FLOW THROUGH A PLANE SLOPE: APPROXIMATE SOLUTION}

It will be useful to obtain an approximate solution to the problem solved exactly in the previous section. For this purpose we shall use the simplification of Jeffreys (1938), Bullard (1938), and Birch (1950), in which the irregular topographic surface is replaced by a plane reference surface whose temperature varies locally in proportion to the topographic relief.

To evaluate the topographic disturbance to temperature by this model at a point whose horizontal coordinate is $x_{0}$, and depth beneath the real surface is $z$, we pass the reference plane through $\left(x_{0}, 0\right)$ and assign to it the temperature:

$$
T(x)=G h(x),
$$

where $G$ is the regional thermal gradient and $h(x)$ is the elevation of the topographic surface relative to the reference plane. (For simplic- 
ity, the topographic surface is considered isothermal in this part of the discussion.) By treating the vertical gradient as uniform in the topographic irregularities, the approximation neglects the effects of heat escaping horizontally through the sloping surfaces.

The temperature disturbance, $\Delta \theta$, is given by a well-known result from potential theory (for example, Birch, 1950, eq 13):

$$
\Delta \Theta\left(x_{0}, z\right)=\frac{1}{\pi} \int_{-\infty}^{+\infty} T(x) \frac{z d x}{\left(x-x_{0}\right)^{2}+z^{2}} .
$$

Formal differentiation of equation 16 yields an expression for the disturbance to the thermal gradient.

$$
\frac{\partial \Delta \theta}{\partial z}=\frac{1}{\pi} \int_{-\infty}^{+\infty} T(x) \frac{\left(x-x_{n}\right)^{2}-z^{2}}{\left[\left(x-x_{0}\right)^{2}+z^{2}\right]^{2}} d x .
$$

At the surface, $z=0$, the gradient disturbance approaches:

$$
\left.\frac{\partial \Delta \Theta}{\partial z}\right|_{z=0}=\frac{1}{\pi} \int_{-\infty}^{+\infty} \frac{T(x)}{\left(x-x_{0}\right)^{2}} d x
$$

The last two equations correspond to Jeffreys' (1938) equations 17 and 18. The integrals require special treatment where $\frac{d T}{d x}$ does not exist, but the mathematics is consistent with physical intuition.

If $G g(x)$ and $Q q(x)$ represent the vertical gradient and heat flow at $(x)$, then $g(x)$ and $q(x)$ represent these quantities normalized to the regional values $G$ and $Q$. Assuming positive heat flow in the direction of decreasing $z$, we have:

$$
\left.\frac{1}{G} \frac{\partial \Delta \Theta}{\partial z}\right|_{z=0}=g(x)-\frac{G}{G_{0}}=q(x)-\frac{Q}{Q_{0}}=\Delta q(x)=\Delta g(x) .
$$

( $G_{0}$ and $Q_{0}$, denoting unit gradient and flux, are introduced for dimensional consistency.) Throughout this paper, "heat-flow anomaly" will refer to the normalized quantity, $\Delta q(x)$, which can be used interchangeably with $\Delta g(x)$, the normalized gradient anomaly. By "heat flow" we shall always mean the normalized vertical heat flow:

$$
q(x)=1+\Delta q(x)
$$

Equation 18 can be written:

$$
\Delta q\left(x_{0}\right)=\frac{1}{\pi G} \int_{-\infty}^{+\infty} \frac{T(x)}{\left(x-x_{0}\right)^{2}} d x
$$


Note that equations 17,18 , and 20 can still be considered exact, provided we consider $T$ as that function which properly represents the topographic relief at the reference plane.

Applying the Jeffreys assumption (eq. 15) yields an approximation for $\Delta q$ which we denote by $\Delta q^{\prime}$.

$$
\Delta q^{\prime}\left(x_{0}\right)=\frac{1}{\pi} \int_{-\infty}^{+\infty} \frac{h(x)}{\left(x-x_{0}\right)^{2}} d x .
$$

$\Delta q^{\prime}$, and $q^{\prime}=1+\Delta q^{\prime}$ will be referred to as the "Jeffreys approximation" to $\Delta q$ and $q$, respectively.

For a plane slope of height $H$ and angle $\beta$ we have:

$$
\begin{aligned}
h(x) & =H, x>0 \\
& =H+x \tan \beta, 0>x>-H \cot \beta \\
& =0,-H \cot \beta>x .
\end{aligned}
$$

Substituting equations 22 in equation 21 yields:

$$
\begin{aligned}
q^{\prime}(x)=1+\Delta q^{\prime}(x) & =1+\frac{1}{\pi} \tan \beta \ln \left(\frac{x}{x+H \cot \beta}\right) \text { where } 0<\beta<\frac{\pi}{2} \\
& =1-\frac{1}{\pi} \frac{H}{x} \text { where } \beta=\frac{\pi}{2}
\end{aligned}
$$

Equations 23 apply for all $x$ except the singular points $x=0$ and $x=-H \cot \beta$. They represent an approximation to $q$ which is given exactly by equations 12,13 , and 14 . The two quantities $q$ and $q^{\prime}$ are compared for selected values of $\beta$ in figure 2 and their difference:

$$
D=q-q^{\prime},
$$

is illustrated in figures 5 and 6 and tabulated in tables 1,2 , and 3.

\section{COMPARISON OF THE EXACT AND APPROXIMATE SOLUTIONS}

From figure 2 it is seen that the approximation based on the Jeffreys assumption is generally good for points beyond the toe or behind the brink of a plane slope if the angle is less than $30^{\circ}$. For angles less than about $9^{\circ}$ it is also rather good for points on the sloping surface unless these points lie extremely close to the toe or brink. Inasmuch as the Jeffreys approximation can be applied to general topographic forms and the exact solution applies only to the plane slope, the difference between them will be investigated in an attempt to find better ways to represent the surface heat flow from more general topography. 


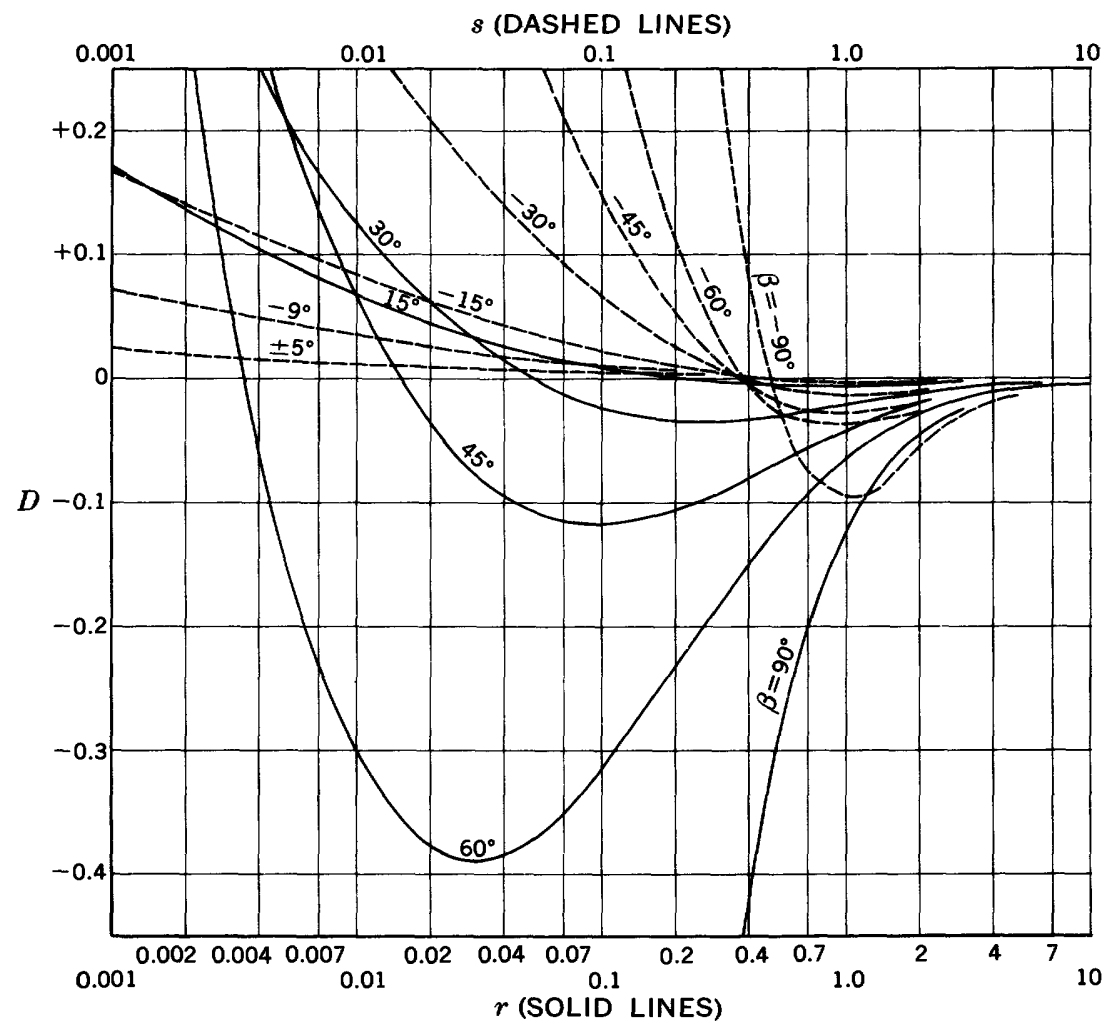

Figure 5.-Difference $(D)$ between the exact solution and the Jeffreys approximation to the heat flow beyond the toe (solid lines) and behind the brink (dashed lines) of a plane slope for various angles $(\beta)$.

Figure 7 compares the temperature distribution over the reference plane assumed in the approximate solution (solid lines) with a schematic representation of the distribution required by the exact solution (dashed lines) for gradient calculations at the point $x_{0}$ beyond the toe (fig. $7 A$ ), on the slope (fig. $7 B$ ), and behind the brink (fig. $7 C$ ). The two reference temperatures differ by a factor denoted by $(1+\epsilon)$. At large distances from the slope, $\epsilon$ vanishes, and the two representations of the reference-plane temperature coincide. The difference in the two representations is the effect (neglected in the approximation) of lateral variation in vertical gradient caused by the topographic relief. Thus $\epsilon$ can be viewed as the effect of interaction of the slope with itself. For example, near $x=0$ in figure $7 A$, the vertical heat flow is lower than the regional average and hence so is the vertical gradient. Thus the reference-plane temperature is lower than anticipated by the approximation. The reverse is true near the toe of the slope (fig. $7 \mathrm{~A}$ ). In figure $7 \mathrm{C}$ where the reference plane is above the 


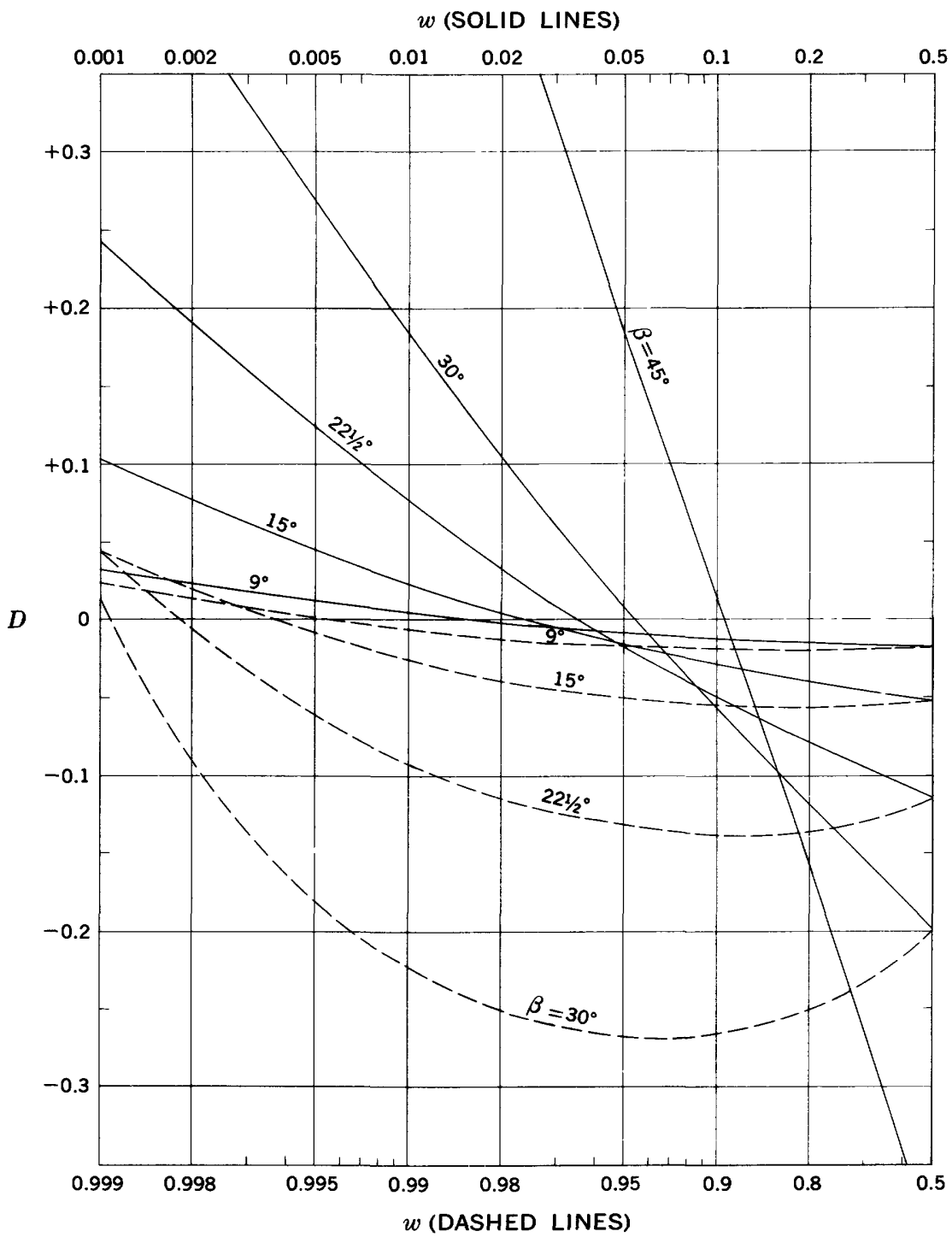

Figure 6.-Difference $(D)$ between the exact solution and the Jeffreys approximation to vertical heat flow on the inclined part of a plane slope. Upper half of the slope $(w<0.5)$ represented by solid curves and upper scale. Lower half $(w>0.5)$ represented by dashed lines and lower scale. 


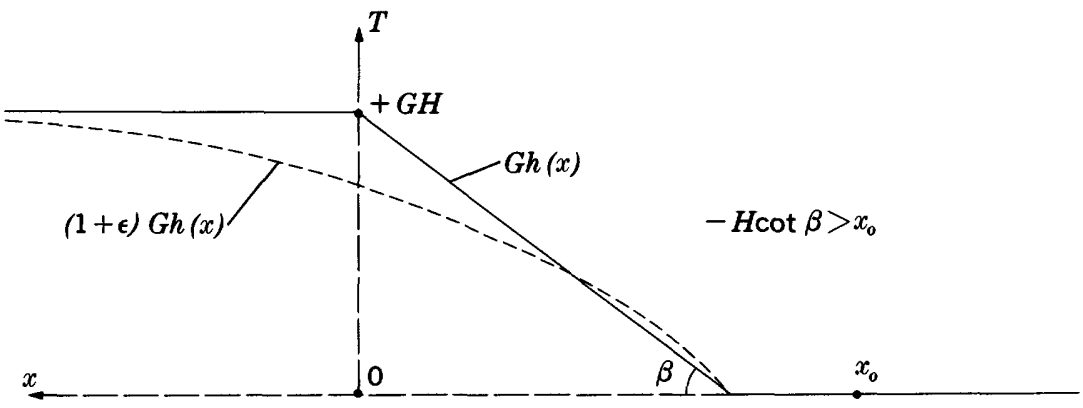

$A$
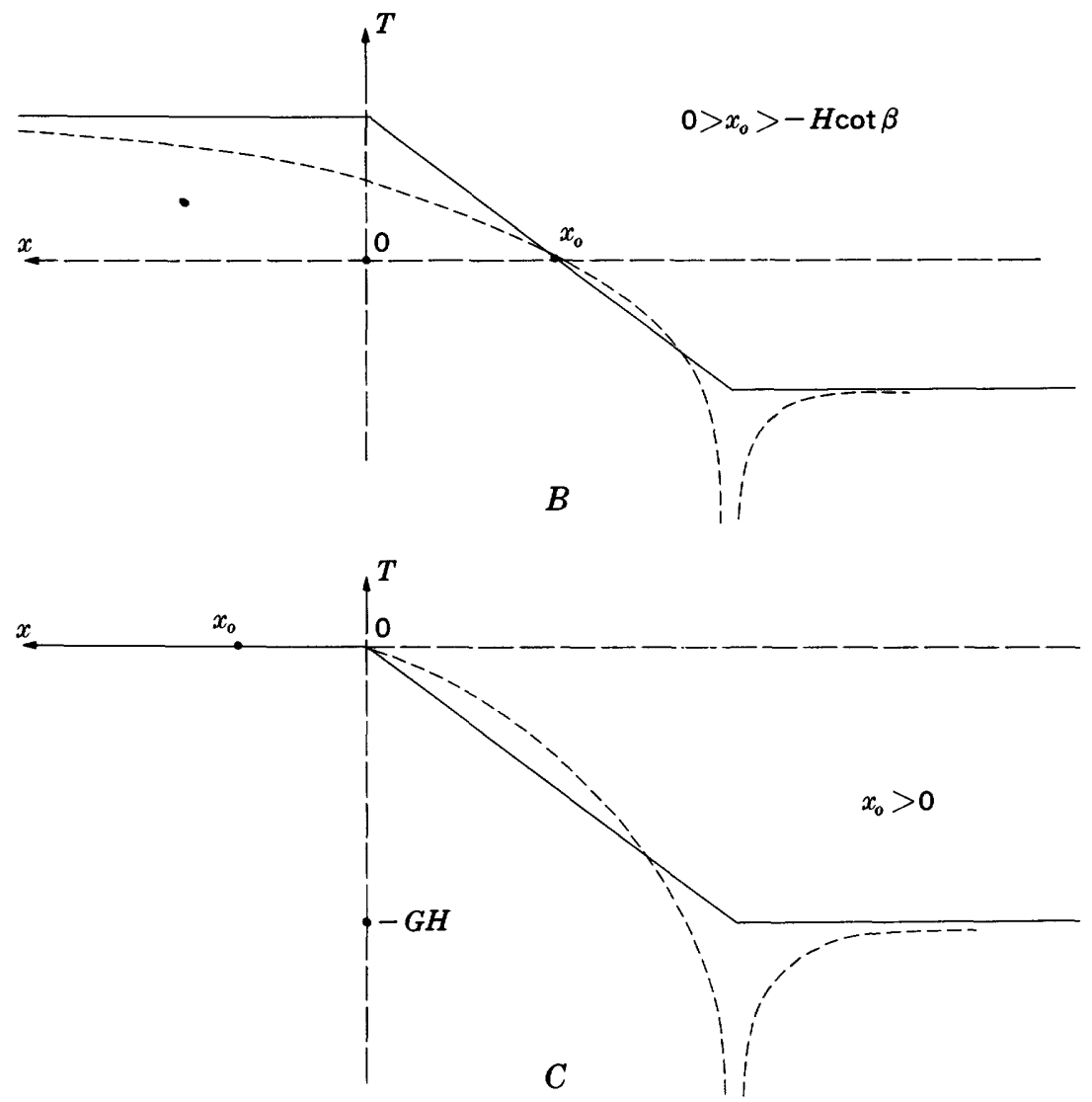

FigURE 7.-Diagrammatic comparison of the reference-plane temperatures required by the exact solution (dashed lines) and Jeffreys approximation (solid lines) for stations beyond the toe $(A)$, on the incline $(B)$, and behind the brink $(C)$ of a plane slope. 
topographic surface, high heat flow near the toe results in large gradients and low reference-plane temperatures. Setting $\Delta q=q(x)-1$, we can write an exact expression analogous to equation 21:

$$
\begin{gathered}
\Delta q\left(x_{0}\right)=\frac{1}{\pi} \int_{-\infty}^{+\infty} \frac{h(x)[1+\epsilon]}{\left(x-x_{0}\right)^{2}} d x \\
=\Delta q^{\prime}(x)+\frac{1}{\pi} \int_{-\infty}^{+\infty} \frac{h(x) \epsilon}{\left(x-x_{0}\right)^{2}} d x \\
\text { or } D=\Delta q-\Delta q^{\prime}=\frac{1}{\pi} \int_{-\infty}^{+\infty} \frac{h(x) \epsilon}{\left(x-x_{0}\right)^{2}} d x .
\end{gathered}
$$

It is seen that $\epsilon(x)$ can be viewed (for positive $h(x)$ ) as the mean value of the gradient disturbance at $x$ between the topographic surface and the reference plane or the mean value of the heat-flow disturbance there. For negative $h$ it is, of course, a fictitious quantity but nevertheless a useful intuitive concept.

The difference, $D$, between the exact and approximate solutions at points on the horizontal surfaces is illustrated for various slope angles in figure 5. For angles less than $90^{\circ}$ the Jeffreys approximation underestimates the heat flow near the toe (positive $D$ ) and at greater distances overestimates it (fig. 5, negative $D$ ). Positive $D$ for small $r$ is the second-order effect of the concentration of vertical flux by the slope near its toe. It can be viewed as a locsl effect of the positive portion of $\epsilon$ in figure $7 \mathrm{~A}$. At larger distances the exact solution yields lower heat flows than the Jeffreys approximation because of the general lowering of reference-plane temperature caused by horizontal escape of heat from the sloping surface.

The positive values of $D$ for small $s$ are the local effects of reduced heat flow near the brink which increases reference-plane temperatures there when it operates through negative topography (fig. $7 C$ ). For larger $s, D$ is negative because of horizontal losses. Figure 6 illustrates $D$ for points on the sloping surface. It is positive near the toe and brink and negative in the central portion, as one would anticipate from the above discussion.

From equations 23 it is seen that $\Delta q^{\prime}$ is symmetrical about the midpoint of the slope $(x=-0.5 H \cot \beta)$ with singularities at the brink and toe. The exact solution is asymmetrical because $\epsilon$ is asymmetrical; the negatively infinite heat flow at the brink becomes zero heat flow in the exact solution, and heat flows are below the regional value over most of the slope. At the midpoint where $q^{\prime}$ is unity, the exact heat-flow anomaly, $\Delta q$, is given to a very good approximation by:

$$
\Delta q \cong-0.8 \sin ^{2} \beta, x=-0.5 H \cot \beta \text {. }
$$


It is clear from the figures and tables discussed, that the difference, $D$, between $\Delta q^{\prime}$ and $\Delta q$ generally decreases with decreasing slope angle, $\beta$, and increasing distance from the slope.

\section{THE PROBLEM OF THE GENERAL TWO-DIMENSIONAL SLOPE}

We should like to apply the results obtained so far to the rapid estimation of surface heat-flow anomalies caused by real, roughly two-dimensional slopes on the earth's surface. At best, real slopes might resemble the plane-slope model, but they will never correspond to it in detail. Generally, real slopes will have distinguishing features such as humps, hollows, and rounded corners. Even very small departures from the model might cause very large errors in the heatflow estimate if they occur near the station. This situation is clear when it is considered that the superficial effect of a $2-\mathrm{km}$ (kilometer) slope $1 \mathrm{~km}$ from the station is equivalent to that of a $10-\mathrm{m}$ (meter) slope of the same shape $5 \mathrm{~m}$ from the station. (In the case of the latter slope, the effect would fall off more rapidly with depth.) If both slopes occurred together, their combined effect could not be accounted for with confidence by adding the exact solutions for the individual components, because each slope would affect the heat flow through the other and alter its effect on the heat flow at the station. Such interaction precludes the general superposition of plane slopes. It is possible to perform calculations like those of the first section to obtain the exact solution for two coexisting plane slopes, but the results would contain four parameters instead of two, and could not be presented in any simple way for rapid graphical computations. Furthermore, we would be little better off as far as real topography is concerned.

Unlike the exact solution, $\Delta q$, the Jeffreys approximation, $\Delta q^{\prime}$, can be obtained easily for any surface shape by adding individual contributions of the topographic elements. It is clear that it is a good approximation under some conditions, but as we have already seen, it can also be very much in error. By approximating the effect of a plane slope by the sum of exact effects of smaller plane slopes of the same angle, it can be shown that neither the Jeffreys approximation nor the summing of exact solutions is consistently better as an approximating scheme. It might be suspected that if a general slope were decomposed into plane-slope components so small that the sum of their $D$ 's was negligible, then their individual exact contributions could be added to obtain the effect of the general slope. However it can be shown that such an approximation approaches the Jeffreys result as the components become small. It can be seen from the change of sign of $D$ with $x$ (figs. 5 and 6 ), that neither of these approximation schemes can even be relied upon to give a limit (upper or lower) to 
the heat-flow anomaly from a general slope. Therefore, in the sections that follow, more indirect methods will be used to apply the results for a plane slope to estimate the effects of more complicated topographic relief.

\section{HEAT FLUX ON A HORIZONTAL SURFACE BETWEEN TWO PLANE SLOPES}

It has been pointed out that the plane slope is a highly idealized topographic form, but that more complicated exact models generally lose the advantage of the two-parameter representation or of intuitive simplicity. Extending the plane-slope results to characterize more general configurations is therefore worthwhile, and can be done with limited success for the heat flux on a horizontal surface between two plane slopes $\left(h_{a}(x)\right.$ and $h_{b}(x)$, fig. 8$)$. There are three cases. In the first (fig. $8 A$ ), the station lies on the horizontal surface between two positive plane slopes. We shall call this the plane valley. In the second case, the plane ridge (fig. $8 B$ ), the station lies on the horizontal surface between two negative plane slopes. The third case is the plane bench (fig. $8 \mathrm{C}$ ) in which the station lies on the horizontal surface between plane slopes of opposite sign. In presenting the general theory we shall refer to the example of case 1.

Consider the half space $z>0$ upon whose surface $z=0,-\infty<x<+\infty$ are placed two plane slopes $h_{a}(x)$ and $h_{b}(x)$ as illustrated in figure 9 . We denote the topographic surface as $h(x)$ :

$$
\begin{aligned}
h(x) \equiv h_{a}(x)+h_{b}(x) & =h_{a}(x), x>a \\
& =0, a<x<b \\
& =h_{b}(x), b>x .
\end{aligned}
$$

We shall consider the surface həat flux at a point $x_{0}$ on the horizontal surface - strip $a>x>b$. The heat-flow anomaly, $\Delta q\left(x_{0}\right)$, cannot be obtained by simply adding the heat-flow anomaly $\Delta q_{a}\left(x_{0}\right)$ and $\Delta q_{b}\left(x_{0}\right)$ of the independent plane slopes because the presence of $h_{a}$ modifies the heat flow through $h_{b}$ (and conversely) and this modification, in turn, further modifies the heat flow through $h_{a}$ and so on. There is, however, a hypothetical temperature distribution, $T(x)$, over the plane $z=0$ that will affect the heat flow at $x_{0}$ in the same way as the isothermal topographic surface $h(x)=h_{a}(x)+h_{b}(x)$. Although the plane slopes are not superimposable in the geometric sense, their collective contributions to the reference-plane temperature are. We therefore represent the reference-plane temperature:

$$
\begin{aligned}
\frac{1}{G} T(x)=h_{a}\left[1+\epsilon_{a}+\right. & \left.\delta q_{b a}\left(1+e_{b a}\right)+\delta q_{a b a}\left(1+e_{a b a}\right)+* * *\right] \\
& +h_{b}\left[1+\epsilon_{b}+\delta q_{a b}\left(1+e_{a b}\right)+\delta q_{b a b}\left(1+e_{b a b}\right)+* * *\right] .
\end{aligned}
$$




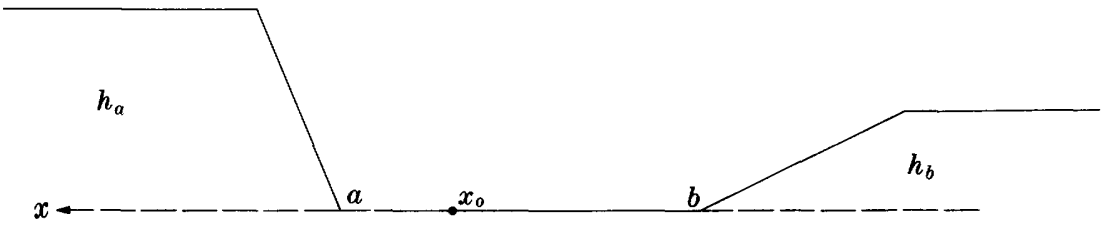

$A$

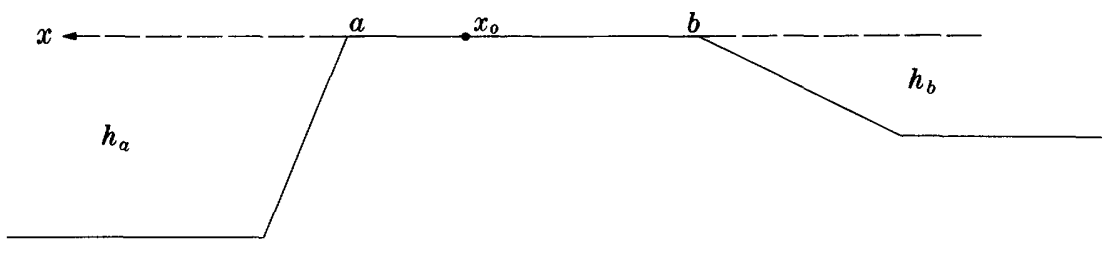

$B$

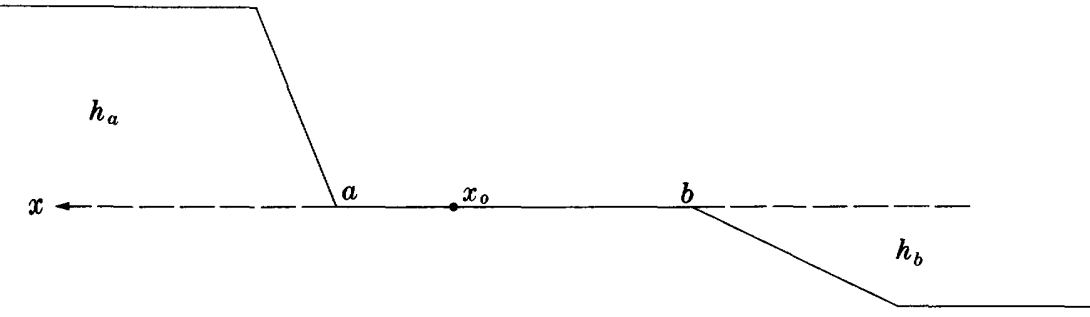

$C$

Figure 8.-Coexisting plane slopes: $A$, a plane valley, $B$, a plane ridge, and $C$, a plane bench.

Taking only the first term in each series yields the simple Jeffreys approximation to the reference-plane temperature. The second terms have the same meaning as $\epsilon$ in equation 24 (fig. $9 A, B$ ). These terms adjust the Jeffreys approximation for the independent variation in vertical gradient within each topographic element; that is, they adjust for the interaction of each slope with itself. Approximating $T(x)$ by the first two terms of each series is equivalent to approximating 

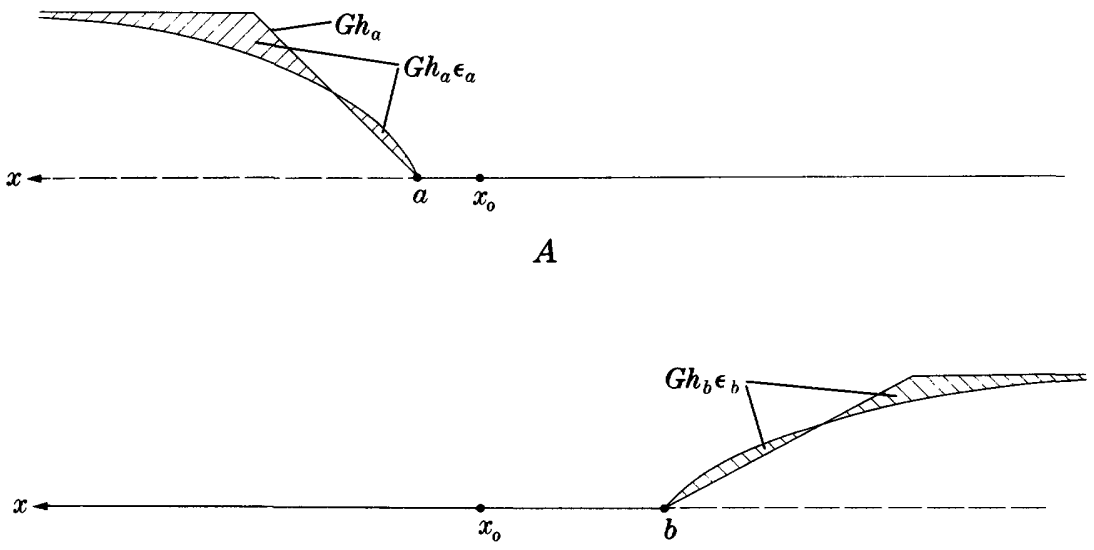

$B$

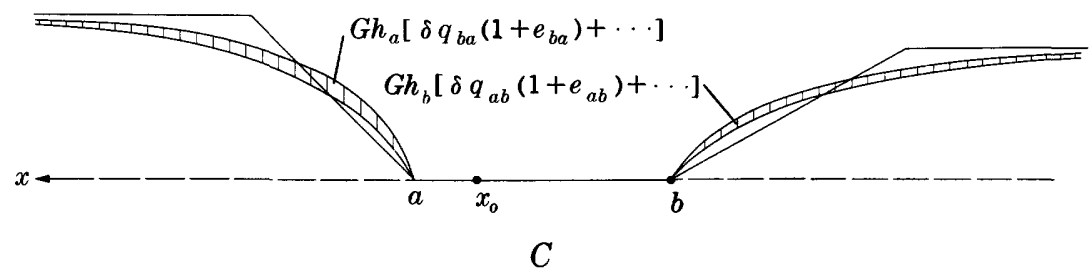

FrguRE 9.-Geometrical significance of the leading terms $(A, B)$ of equation 29 and the interaction terms $(C)$ for the plane valley.

$q\left(x_{0}\right)$ by the sum of the exact solutions for the effects of $h_{a}$ and $h_{b}$ independently. The term with subscript " $b a$ " is the effect of the firstorder interaction of $h_{b}$ with $h_{a} . \delta q_{b a}$ is the additional flux that would result across the reference plane beneath $h_{a}$ if $h_{a}$ were not there. $e_{\partial a}$ plays a role similar to $\epsilon_{a}$, adjusting the reference-plane temperature for the interaction of the flux increment $\delta q_{b a}$ with the slope $h_{a}$ (fig. $9 C$ ). It is represented by a different symbol because we have no theory to evaluate it, inasmuch as $\delta q_{b a}$ is a function of $x$. Similarly the " $a b$ " term represents the first-order interaction effect of $h_{a}$ on the reference-plane temperature beneath $h_{b}$. The flux increment $\delta q_{a b}$ increases the reference-plane temperature beneath $h_{b}$ and this in turn increases the flux through $h_{a}$ by an amount $\delta q_{a b a}$. and increases the reference-plane temperature there by $G h_{a} \delta q_{a b a}\left(1+e_{a b a}\right)$, and so on. Neglecting the " $e$ " in the interaction terms results in a Jeffreys-type approximation to them.

For convenience equation 29 is rewritten:

$$
T(x)=T_{a}+T_{b a}+T_{a b a}+T_{b a b a}+_{* * *}+T_{b}+T_{a b}+T_{b a b}+T_{a b a b}+_{* * *}
$$


where

$$
T_{a}=G h_{a}\left(1+\epsilon_{a}\right), T_{b}=G h_{b}\left(1+\epsilon_{b}\right) .
$$

$$
T_{b a}=G h_{a} \delta q_{b a}\left(1+e_{b a}\right), T_{a b}=G h_{b} \delta q_{a b}\left(1+e_{a b}\right) \text {. }
$$

$$
T_{* * * a}=G h_{a} \delta q_{* * * a}\left(1+e_{* * * a}\right), T_{* * * b}=G h_{b} \delta q_{* * * b}\left(1+e_{* * * b}\right) .
$$

The asterisks represent an arbitrary number of alternating subscripts. The terms with multiple subscripts will be referred to as interaction terms, with $T_{b a}$ and $T_{a b}$ being the first-order ones and so on. The terms are all functions of $x$. Increasing any one of them increases $T(x)$, and by equation 21 increases the heat flow.

As long as $h_{a}(x)$ and $h_{b}(x)$ are plane slopes we may replace $\delta q_{b a}(x)$ by $\Delta q_{b}(x)$ and $\delta q_{a b}(x)$ by $\Delta q_{a}(x)$. These quantities decrease in magnitude with increasing distance from $h_{b}$ and $h_{a}$ respectively. (This condition and the analysis that follows are valid even if $h_{a}(x)$ and $h_{b}(x)$ are not plane slopes as long as each is of one sign.) Hence, the greatest absolute value of $\Delta q_{b}(x)$ in $h_{a}$ is attained at the point of closest approach of $h_{a}$ to $h_{b}$, that is, at the toe, $x=a$. Replacing $\delta q_{b a}$ by its maximum absolute value we obtain the inequality:

$$
\left|h_{a} \delta q_{b a}(x)\left(1+e_{b a}\right)\right|<\left|h_{a} \Delta q_{b}(a)\left(1+e_{b a}\right)\right| .
$$

The quantities within the absolute value signs will always be of the same sign.

The appropriate value of $e_{b a}$ on the right side of equation 5-4 is very nearly $\epsilon_{a}$ because of the assignment of the constant value, $\Delta q_{b}(a)$, to the heat increment. This substitution is largely a matter of convenience, as the $e$ is of higher order than the $\Delta q$. Thus:

$$
\begin{aligned}
& \left|T_{b a}(x)\right|<\left|G h_{a} \Delta q_{b}(a)\left(1+\epsilon_{a}\right)\right|=\left|\Delta q_{b}(a) T_{a}\right| . \\
& \left|T_{a b}(x)\right|<\left|G h_{b} \Delta q_{a}(b)\left(1+\epsilon_{b}\right)\right|=\left|\Delta q_{a}(b) T_{b}\right| .
\end{aligned}
$$

Proceeding to higher order terms with similar reasoning:

$$
\begin{gathered}
\left|T_{a b a}(x)\right|<\left|G h_{a} \Delta q_{a}(b) \Delta q_{b}(a)\left(1+\epsilon_{a}\right)\right|=\left|\Delta q_{a}(b) \Delta q_{b}(a) T_{a}\right|, \\
\left|T_{b a b}(x)\right|<\left|G h_{b} \Delta q_{b}(a) \Delta q_{a}(b)\left(1+\epsilon_{b}\right)\right|=\left|\Delta q_{b}(a) \Delta q_{a}(b) T_{b}\right|, \\
\left|T_{b a b a}(x)\right|<\left|G h_{a} \Delta q_{b}(a) \Delta q_{a}(b) \Delta q_{b}(a)\left(1+\epsilon_{a}\right)\right|=\left|\Delta q_{b}(a) \Delta q_{a}(b) \Delta q_{b}(a) T_{a}\right|, \\
\left|T_{a b a b}(x)\right|<\left|G h_{b} \Delta q_{a}(b) \Delta q_{b}(a) \Delta q_{a}(b)\left(1+\epsilon_{b}\right)\right|=\left|\Delta q_{a}(b) \Delta q_{b}(a) \Delta q_{a}(b) T_{b}\right|,
\end{gathered}
$$

with similar expressions for terms of higher order.

Denoting the quantities in absolute value signs on the right side of equations $32-37$ with a prime we have in general:

and

$$
\left|T_{* * * a}(x)\right|<\left|T_{* * * a}^{\prime}(x)\right|
$$

$$
\left|T_{* * * b}(x)\right|<\left|T_{* * * b}^{\prime}(x)\right| \text {. }
$$


The corresponding primed and unprimed T's will always be of the same sign.

The series of equation 30 is dominated term by term by:

$$
\left|T_{a}\right|+\left|T^{\prime}{ }_{b a}\right|+\left|T^{\prime}{ }_{a b a}\right|+\left|T^{\prime}{ }_{b a b a}\right|+*_{*} *+\left|T_{b}\right|+\left|T^{\prime}{ }_{a b}\right|+\left|T^{\prime}{ }_{b a b}\right|+\left|T^{\prime}{ }_{a b a b}\right|++_{* *},
$$

which by equations $32-37$ can be written:

$$
\begin{aligned}
& \left|T_{a}\right|\left[1+\left|\Delta q_{b}(a)\right|+\left|\Delta q_{a}(b) \Delta q_{b}(a)\right|+\left|\Delta q_{b}(a) \Delta q_{a}(b) \Delta q_{b}(a)\right|++_{* *}\right] \\
& +\left|T_{b}\right|\left[1+\left|\Delta q_{a}(b)\right|+\left|\Delta q_{b}(a) \Delta q_{a}(b)\right|+\left|\Delta q_{a}(b) \Delta q_{b}(a) \Delta q_{a}(b)\right|+*_{* *}\right] .
\end{aligned}
$$

And this converges if:

and

$$
\begin{array}{r}
\Delta q_{a}(b)<1 \\
\Delta q_{b}(a)<1 .
\end{array}
$$

Therefore the series of equation 30 is absolutely convergent under condition 39. (Absolute value signs are not needed in condition 39, as $\Delta q$ can never be less than -1 except in overhanging topography. At a projecting corner its value is equal to -1 , and as this value is known we shall never want to calculate it.) No attempt will be made to establish rigorously that the representation of $T(x)$ in equation 30 actually converges to the required limit. In general if $\delta q_{a b}$ and $\delta q_{b a}$ are not too large it can be expected to converge rapidly and to yield an excellent approximation.

Relations 38 apply whenever $T(x)$, equation 30 represents the temperature on a horizontal plane separating two plane slopes. It has been pointed out that there are three cases. We shall now consider them individually.

\section{CASE 1, PLANE VALIEY $\left(h_{a}(x) \geq 0, h_{b}(x) \geq 0\right)$}

In this case $h_{a}$ can only increase the heat flow through $h_{b}$, and conversely. Hence the interaction terms of equation 30 are all positive and so are their primed approximations relations 38 . Therefore, neglecting the interaction terms yields a lower limit to the heat-flow anomaly, $\Delta q\left(x_{0}\right)$, and replacing them by the primed terms yields an upper limit.

Using equation 21 :

$$
\begin{gathered}
\Delta q\left(x_{0}\right)>\frac{1}{\pi G} \int_{-\infty}^{+\infty} \frac{T_{a}+T_{b}}{\left(x-x_{0}\right)^{2}} d x \\
\Delta q\left(x_{0}\right)<\frac{1}{\pi G} \int_{-\infty}^{+\infty}\left\{\left[T_{a}+T_{b a}^{\prime}+T_{a b a}^{\prime}+^{* * *}\right]\right. \\
\left.+\left[T_{b}+T_{a b}^{\prime}+T^{\prime}{ }_{b a b}+* * *\right]\right\} \frac{d x}{\left(x-x_{0}\right)^{2}}
\end{gathered}
$$




$$
\begin{aligned}
& =\frac{1}{\pi} \int_{-\infty}^{+\infty}\left\{T_{a}\left[1+\Delta q_{b}(a)+\Delta q_{a}(b) \Delta q_{b}(a)+{ }^{* *}\right]+T_{b}\left[1+\Delta q_{a}(b)\right.\right. \\
& \left.\left.+\Delta q_{b}(b) \Delta q_{a}(b)+* * *\right]\right\} \frac{d x}{\left(x-x_{0}\right)^{2}},
\end{aligned}
$$

but:

$$
\begin{aligned}
& \frac{1}{\pi G} \int_{-\infty}^{+\infty} \frac{T_{a}}{\left(x-x_{0}\right)^{2}} d x=\frac{1}{\pi} \int_{-\infty}^{+\infty} \frac{h_{a}\left(1+\epsilon_{a}\right)}{\left(x-x_{0}\right)^{2}} d x=\Delta q_{a}\left(x_{0}\right), \\
& \frac{1}{\pi G} \int_{-\infty}^{+\infty} \frac{T_{b}}{\left(x-x_{0}\right)^{2}} d x=\frac{1}{\pi} \int_{-\infty}^{+\infty} \frac{h_{b}\left(1+\epsilon_{b}\right)}{\left(x-x_{0}\right)^{2}} d x=\Delta q_{b}\left(x_{0}\right),
\end{aligned}
$$

and finally:

$$
\begin{aligned}
\Delta q_{a}\left(x_{0}\right) & +\Delta q_{b}\left(x_{0}\right)<\Delta q\left(x_{0}\right) \\
< & \Delta q_{a}\left(x_{0}\right)\left[1+\Delta q_{b}(a)+\Delta q_{a}(b) \Delta q_{b}(a)+\Delta q_{b}(a) \Delta q_{a}(b) \Delta q_{b}(a)+{ }^{* *}\right] \\
& +\Delta q_{b}\left(x_{0}\right)\left[1+\Delta q_{a}(b)+\Delta q_{b}(a) \Delta q_{a}(b)+\Delta q_{a}(b) \Delta q_{b}(a) \Delta q_{a}(b)+* * *\right] .
\end{aligned}
$$

The application of inequality 44 is illustrated with a few simple numerical examples.

Example 1.-Suppose $h_{a}$ and $h_{b}$ are both $45^{\circ}$ slopes of equal height $(H)$ separated by a distance $(a-b)$ of twice their height $(2 H)$. To find the heat flow at the midpoint we need the following from table 1 :

$$
\begin{gathered}
\Delta q_{a}(b)=\Delta q_{b}(a)=\Delta q\left(45^{\circ}, r=2\right)=0.108 \\
\Delta q_{a}\left(x_{0}\right)=\Delta q_{b}\left(x_{0}\right)=\Delta q\left(45^{\circ}, r=1\right)=0.178 .
\end{gathered}
$$

Then by inequality 44 :

$$
0.178+0.178<\Delta q\left(x_{0}\right)<[0.178+0.178][1+0.108+0.012] .
$$

\section{Hence}

or equivalently,

$$
0.356<\Delta q\left(x_{0}\right)<0.399 \text {, }
$$

$$
1.36<q\left(x_{0}\right)<1.40 \text {. }
$$

The corresponding result from the Jeffreys approximation is 1.44 .

Example 2.-Taking the same configuration as in example 1, we now consider $x_{0}$ to be $0.1 H$ from $h_{a}$ and $1.9 H$ from $h_{b}$. Since the configuration is unchanged, the series is the same as in the previous example-its sum being 1.120. But now:

$$
\begin{aligned}
& \Delta q_{a}\left(x_{0}\right)=\Delta q\left(45^{\circ}, r=0.1\right)=0.644 \\
& \Delta q_{o}\left(x_{0}\right)=\Delta q\left(45^{\circ}, r=01.9\right)=0.11,
\end{aligned}
$$


where the last value is taken from plate 1 . Hence:

and

$$
0.644+0.11<\Delta q\left(x_{0}\right)<[0.644+0.11][1.120],
$$

$$
1.75<q\left(x_{0}\right)<1.84 \text {. }
$$

In this example $q^{\prime}=1.90$.

Example 3.-Leaving $h_{b}$ and the separation, $a-b$, unchanged we now double the height of $h_{a}$ and change its angle to $30^{\circ}$. Assume $x_{0}$ is midway between the slopes as in example 1. From table 1:

$$
\begin{gathered}
\Delta q_{a}(b)=\Delta q\left(30^{\circ}, r=1\right)=0.162, \\
\Delta q_{a}\left(x_{0}\right)=\Delta q\left(30^{\circ}, r=0.5\right)=0.243, \\
\Delta q_{b}(a)=\Delta q\left(45^{\circ}, r=2\right)=0.108, \\
\Delta q_{b}\left(x_{0}\right)=\Delta q\left(45^{\circ}, r=1\right)=0.178 .
\end{gathered}
$$

Inequality 44 yields:

$0.243+0.178<\Delta q\left(x_{0}\right)<0.243[1+0.108+0.017]+0.178[1+0.162+0.017]$, or equivalently,

$$
1.42<q\left(x_{0}\right)<1.48 \text {. }
$$

In this example the Jeffreys approximation is $q^{\prime}=1.50$.

It is seen that $q^{\prime}$ is a rather good approximation in all these examples. This is partly due to the fact that over the useful range of $r$ and $\beta$ for inequality 44, $D$ is negative (fig. 5 ), and this tends to compensate for the positive interaction terms in the inequality.

CASE 2, PLANE RIDGE $\left(h_{a}(x) \leq 0, h_{b}(x) \leq 0\right)$

In this case the series of equation 30 alternates in sign as follows:

$$
\begin{gathered}
T(x)=-\left|T_{a}\right|+\left|T_{b a}\right|-\left|T_{a b a}\right|+\left|T_{b a b a}\right|-* * * \\
-\left|T_{b}\right|+\left|T_{a b}\right|-\left|T_{b a b}\right|+\left|T_{a b a b}\right|-* * * .
\end{gathered}
$$

The factors $\delta q_{a b}$ and $\delta q_{b a}$ of equation 29 are negative, but their effects on $T(x)$ are positive because $h_{a}$ and $h_{b}$ are negative. The increase in reference-plane temperature $\left(T_{a b}\right.$ and $\left.T_{b a}\right)$ caused by these terms results in the second-order heat increments $\delta q_{n b a}$ and $\delta q_{b a b}$ (eq. 29) being positive with a negative effect on reference-plane temperature $\left(T_{a b a}\right.$ and $\left.T_{b a b}\right)$ and so on.

From the discussion on convergence we know that the sign of the series of interaction terms is the same as that of their leading terms, 
$T_{b a}$ and $T_{a b}$; hence, neglecting interaction gives a lower limit to $\Delta q\left(x_{0}\right)$. Furthermore the error in approximating the interaction is of the same sign as the first term neglected; hence considering only the first-order interaction gives an upper limit.

$$
\begin{gathered}
\Delta q\left(x_{0}\right)>\frac{1}{\pi G} \int_{-\infty}^{+\infty} \frac{T_{a}+T_{b}}{\left(x-x_{0}\right)^{2}} d x^{\prime} . \\
\Delta q\left(x_{0}\right)<\frac{1}{\pi G} \int_{-\infty}^{+\infty}\left(T_{a}+T_{b a}^{\prime}+T_{b}+T_{a b}^{\prime}\right) \frac{d x}{\left(x-x_{0}\right)^{2}}
\end{gathered}
$$

Therefore:

$$
\Delta q_{a}\left(x_{0}\right)+\Delta q_{b}\left(x_{0}\right)<\Delta q\left(x_{0}\right)<\Delta q_{a}\left(x_{0}\right)\left[1+\Delta q_{b}(a)\right]+\Delta q_{b}\left(x_{0}\right)\left[1+\Delta q_{a}(b)\right] .
$$

Example 1.-Assume, as before, that $h_{a}(x)$ and $h_{b}(x)$ are of the same height and are separated by a distance (fig. $8 B, a-b$ ) equal to twice their height. For $x_{0}$ midway between two slopes of $45^{\circ}$ we obtain from table 2:

$$
\begin{gathered}
\Delta q_{a}\left(x_{0}\right)=\Delta q_{b}\left(x_{0}\right)=\Delta q\left(45^{\circ}, s=1\right)=-0.249, \\
\Delta q_{a}(b)=\Delta q_{b}(a)=\Delta q\left(45^{\circ}, s=2\right)=-0.149,
\end{gathered}
$$

and from 46 :

$$
-0.498<\Delta q\left(x_{0}\right)<-0.498[1-0.149]=-0.424,
$$

or equivalently,

$$
0.502<q\left(x_{0}\right)<0.576 \text {. }
$$

The corresponding Jeffreys result $\left(q^{\prime}=0.56\right)$ lies in the bracketing interval.

The good agreement of $q^{\prime}$ is again due in part to the sign of $D$ in these examples; that is, the Jeffreys approximation is better for the combination of slopes than for the slopes individually.

Example 2.-If both slope angles were $5^{\circ}$, the result would be:

In this case $q^{\prime}=0.87$.

$$
0.86<q\left(x_{0}\right)<0.87 \text {. }
$$

Example 3.-Considering the $45^{\circ}$ slopes again, we place $x_{0}$ onetenth $H$ from $a$ and $1.9 H$ from $b$ and obtain:

$$
0.23<q\left(x_{0}\right)<0.34 \text {. }
$$

In this case, $D$ is strongly positive for $\beta=45^{\circ}, s=0.1$ (fig. 5), and hence the Jeffreys approximation $\left(q^{\prime}=0.10\right)$ falls well below the bracketing interval. 
CAgE 3, PLANE BENCH $\left(h_{a}(x) \geq 0, h_{b}(x) \leq 0\right)$

In this case the signs in the series of equation 30 are alternating in groups of two as follows:

$$
\begin{aligned}
T(x)=+\left|T_{a}\right|-\left|T_{b a}\right|-\mid & T_{a b a}|+| T_{b a b a}|+| T_{a b a b a} \mid-* * * \\
& -\left|T_{b}\right|-\left|T_{a b}\right|+\left|T_{b a b}\right|+\left|T_{a b a b}\right|-\left|T_{b a b a b}\right|-* * * .
\end{aligned}
$$

To see this we note that $T_{a}$ and $T_{b}$ must have the same signs as $h_{a}$ and $h_{b}$ respectively. A positive sign for $T_{a}$ leads to a positive heat-flow increment $\delta q_{a b}$ through $h_{b}$ and negative $T_{a b}$ because $h_{b}$ is negative. However, negative $T_{b}$ leads to negative $T_{b a}$, the sign being preserved because $h_{a}$ is positive. Thus any term in the $b$ series leads to a next higher order term of the same sign in the $a$ series, whereas the sign is reversed by negative $h_{b}$ in going from the $a$ series to the next order term in the $b$ series.

By grouping consecutive terms of the same sign it can be seen that the total effect of the interaction in the $a$ series is negative and that $T_{a}$ provides an upper limit and that $T_{a}+T_{b a}+T_{a b a}$ is a lower limit. The lower limit can be replaced by the still smaller but more manageable quantity:

$$
T_{a}+T_{b a}^{\prime}+T_{a b a}^{\prime} .
$$

The grouping shows that a lower limit to the $b$ series (eq 47) is $\mathrm{T}_{b}+T_{a b}$ which can be replaced by the smaller quantity $T_{b}+T^{\prime}{ }_{a b}$.

An upper limit to the $b$ series is:

$$
T_{b}+T_{a b}+T_{b a b}+T_{a b a b}
$$

(We cannot select $T_{b}$ as the upper limit because there is no assurance that the second term dominates the sum of the third and fourth.) To get expression 48 in a manageable form we write:

$$
T_{b}+T_{a b}+T_{b a b}+T_{a b a b}<T_{b}+T_{b a b}+T_{a b a b}<T_{b}+T_{b a b}^{\prime}+T_{a b a b}^{\prime} .
$$

Although deleting $T_{a b}$ weakens the upper limit, it is necessary to do so because we have no assurance that the inequality would be preserved when $T_{a b}$ was replaced by $T_{a b}^{\prime}$. Thus:

$$
\begin{gathered}
\Delta q\left(x_{0}\right)>\frac{1}{G \pi} \int_{-\infty}^{+\infty}\left(T_{a}+T_{b a}^{\prime}+T_{a b a}^{\prime}+T_{b}+T_{a b}^{\prime}\right) \frac{d x}{\left(x-x_{0}\right)^{2}}, \\
\Delta q\left(x_{0}\right)<\frac{1}{G \pi} \int_{-\infty}^{+\infty}\left(T_{a}+T_{b}+T_{b a b}^{\prime}+T_{a b a b}^{\prime}\right) \frac{d x}{\left(x-x_{0}\right)^{2}},
\end{gathered}
$$

and hence:

$$
\Delta q\left(x_{0}\right)>\Delta q_{a}\left(x_{0}\right)\left[1+\Delta q_{b}(a)+\Delta q_{a}(b) \Delta q_{b}(a)\right]+\Delta q_{b}\left(x_{0}\right)\left[1+\Delta q_{a}(b)\right],
$$


and:

$$
\Delta q\left(x_{0}\right)<\Delta q_{a}\left(x_{0}\right)+\Delta q_{b}\left(x_{0}\right)\left[1+\Delta q_{b}(a) \Delta q_{a}(b)+\left(\Delta q_{a}(b)\right)^{2} \Delta q_{b}(a)\right] .
$$

Example.-Assume as before that $h_{a}$ and $h_{b}$ are of equal height $(H)$ and separated by a distance $(a-b)$ of twice this height. If the slope angles are both at $45^{\circ}$ and $x_{0}$ is midway between them, we obtain from tables 1 and 2 :

$$
\begin{gathered}
\Delta q_{a}\left(x_{0}\right)=\Delta q\left(45^{\circ}, r=1\right)=+0.178, \\
\Delta q_{a}(b)=\Delta q\left(45^{\circ}, r=2\right)=+0.108, \\
\Delta q_{b}\left(x_{0}\right)=\Delta g\left(45^{\circ}, s=1\right)=-0.249, \\
\Delta q_{b}(a)=\Delta q\left(45^{\circ}, s=2\right)=-0.149 .
\end{gathered}
$$

By inequality 49a:

$$
\begin{aligned}
\Delta q\left(x_{0}\right)>0.178[1-0.149-0.016]-0.249[1+0.108] \\
=0.178[0.835]-0.249[1.108]=-0.127 .
\end{aligned}
$$

By inequality $49 \mathrm{~b}$ :

$\Delta q\left(x_{0}\right)<+0.178-0.249[1-0.16-0.002]=+0.178$

hence:

$$
-0.249[0.982]=-0.067
$$

$$
0.87<q\left(x_{0}\right)<0.93 .
$$

By comparison $\mathrm{q}^{\prime}=1.00$.

If $x_{0}$ is $1 / 4 H$ from the toe of $h_{a}$ :

$$
1.02<q\left(x_{0}\right)<1.09 \text { and } q^{\prime}=1.19 \text {. }
$$

If it is $1 / 4 H$ from the brink:

$$
0.70<q\left(x_{0}\right)<0.77 \text { and } q^{\prime}=0.81 \text {. }
$$

If $x_{0}$ is $0.1 H$ from the toe of $h_{a}$ and $1.9 H$ from the brink of $h_{b}$ :

$$
1.37<q\left(x_{0}\right)<1.50 \text { and } q^{\prime}=1.63 \text {. }
$$

With $x_{0}$ only $0.1 H$ from the brink:

$$
0.41<q\left(x_{0}\right)<0.51 \text { and } q^{\prime}=0.37 \text {. }
$$

The Jeffreys approximation tends to be too low very close to the brink, but elsewhere on the bench it is generally too high. 
In summary, a lower limit to the heat-flow anomaly on the horizontal surface between two plane slopes $\left(h_{a}\right.$ and $\left.h_{b}\right)$ of the same sign (cases 1 and 2) is provided by the sum of the independent exact solutions for each slope $\left(\Delta q_{a}\left(x_{0}\right)+\Delta q_{b}\left(x_{0}\right)\right)$. The upper limit is provided by adding an overestimate of interaction effects. If the horizontal surface lies between plane slopes of opposite sign (case 3) both the upper and lower limits contain interaction terms, but the sum of the independent exact solutions forms the upper limit to terms of second order in the interaction. In all three cases the bracketing interval $\left(q_{\text {upper }}-q_{\text {lower }}\right)$ is represented by the first-order interaction terms with or without higher order effects.

$$
q_{\text {upper }}-q_{\text {lower }}=\left|\Delta q_{a}\left(x_{0}\right) \Delta q_{b}(a)+\Delta q_{b}\left(x_{0}\right) \Delta q_{a}(b)\right|+\text { higher order. }
$$

Inasmuch as:

hence in all three cases:

$$
\begin{aligned}
& \left|\Delta q_{a}\left(x_{0}\right)\right|>\left|\Delta q_{a}(b)\right|, \\
& \left|\Delta q_{b}\left(x_{0}\right)\right|>\left|\Delta q_{b}(a)\right|,
\end{aligned}
$$

$$
\Delta q\left(x_{0}\right) \approx \Delta q_{a}\left(x_{0}\right)+\Delta q_{b}\left(x_{0}\right)+\left[\Delta q_{a}\left(x_{0}\right) \Delta q_{b}\left(x_{0}\right)\right][1 \pm 1],
$$

where the two signs in the last factor yield upper and lower limits. Relation 51 forms a useful criterion for neglecting interaction. As the slopes become smaller or farther apart, the bracketing intervals become small, and the heat flow is given to a good approximation by the sum of the independent exact solutions $\left[\Delta q_{a}\left(x_{0}\right)+\Delta q_{b}\left(x_{0}\right)\right]$ in all three cases. In all these cases the linear approximation gives surprisingly good results over a wide variety of conditions. It can, however, contain considerable error at points very near steep slopes.

\section{GEOMETRIC BRACKETING}

Consider a slope, $h_{a}(x)$, to which is added a bump, $h_{b}(x)$, such that:

$$
\begin{gathered}
h_{b}(x)>0, x_{1}<x<x_{2}, \\
h_{b}(x)=0, x>x_{2}, x_{1}>x .
\end{gathered}
$$

Uniform heat flow at depth is assumed as before; hence the vertical flux at the surface is positive except at projecting corners where it is zero (fig. 10).

If $\Delta q_{a}(x)$ represents the vertical surface flux through $h_{a}$ when $h_{a}$ is isothermal and $\Delta q_{a b}(x)$, the vertical flux through $h_{a}+h_{b}$ when it is held isothermal, then:

$$
\Delta q_{a b}(x)>\Delta q_{a}(x), x>x_{2}, x_{1}>x .
$$




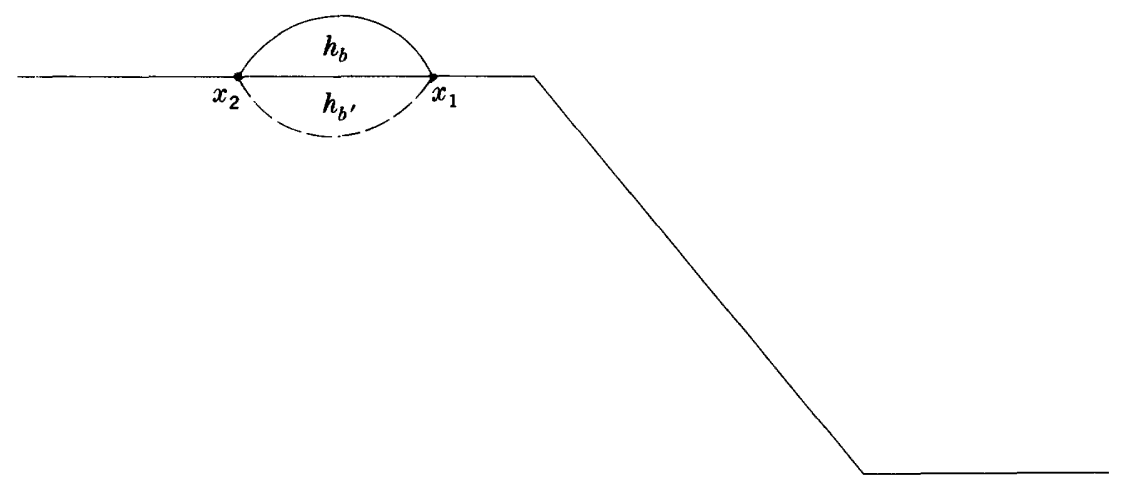

F1Gure 10.-Plane slope with a positive (solid curve) or negative (dashed curve) bump.

This can be seen by noting that for points not in $h_{b}$, the isothermal surface $h_{a}+h_{b}$ can be replaced by $h_{a}$ isothermal outside of $x_{1}<x<x_{2}$ and with some positive temperature distribution over the part of the surface $x_{1}<x<x_{2}$. This temperature excess can be replaced by a distribution of doublets, positive downward, which in turn will increase the vertical flux at all points outside of $h_{b}$ on $h_{a}$.

If $h_{b}{ }^{\prime}$ is a negative bump (fig. 10), the equivalent temperature distribution on $h_{a}$ in $x_{1}<x<x_{2}$ is generally negative. The resulting bowing down of the isotherms in $x_{1}<x<x_{2}$ will result in a convergence of flux there that can only result in a decrease in vertical flux at all points on $h_{a}$ outside of $h_{b}$.

More generally, let $h(x)$ be a general surface and $h_{u}(x)$ and $h_{l}(x)$ be two other surfaces such that:

$$
h_{u}(x) \geqslant h(x) \geqslant h_{l}(x),+\infty>x>-\infty .
$$

The heat-flow anomalies on each surface are denoted respectively by $\Delta q_{u}(x), \Delta q(x)$, and $\Delta q_{\imath}(x)$. Then, at any point $x_{0}$ (not a sharp corner) at which:

$$
h_{u}\left(x_{0}\right)=h\left(x_{0}\right)
$$

we have,

$$
\Delta q_{u}\left(x_{0}\right)>\Delta q\left(x_{0}\right),
$$

and where,

$$
h\left(x_{0}\right)=h_{l}\left(x_{0}\right),
$$

then,

$$
\Delta q\left(x_{0}\right)>\Delta q_{l}\left(x_{0}\right) .
$$


It follows that where,

we have,

$$
h_{u}\left(x_{0}\right)=h\left(x_{0}\right)=h_{l}\left(x_{0}\right),
$$

$$
\Delta q_{u}\left(x_{0}\right)>\Delta q\left(x_{0}\right)>\Delta q_{l}\left(x_{0}\right) .
$$

Relations 53-56 will be referred to as the theorem on geometric bracketing. It is illustrated in figure 11 where $h(x)$ is represented by the horizontal lines, $O L$ and $U^{\prime} P$, and the wavy line $\left(L t_{1} t_{2} U^{\prime}\right)$ joining them. The plane slope $O U U^{\prime} P$ represents $h_{u}$ and the plane slope $O L L^{\prime} P$ represents $h_{l}$. Then relation 56 applies for all points $\left(x_{0}\right)$ on $O L$ and $U^{\prime} P$. One-sided limits are given at $x_{0}=t_{1}$ (relation 54) and at $x_{0}=t_{2}$ (relation 55).

The results of this section can be applied to those of the last section to establish limits to the heat-flow anomaly at stations interior to many real valleys, ridges, and benches.

The method of geometric bracketing can lead quickly to a determination of whether or not specific topographic features are significant in heat-flow studies. For example, the effect of any positive feature is overestimated by that of a cliff of the same height and distance from the station. Thus a positive feature whose height is less than 10 percent of its distance from the station cannot affect the heat flow there by more than 2.8 percent (table 1); if its height is 5 percent of the distance the limit is $1 \frac{1}{2}$ percent. The corresponding limits for negative features are 3.6 and 1.7 percent. (It is surprising that the anomaly 10 slope heights from the toe of a $90^{\circ}$ cliff, 2.8 percent, is not very different from the anomaly 10 slope heights from the toe of a $5^{\circ}$ slope, 2.1 percent, table 1.) If features of the same sign occur at such distances on both sides of the station, their interaction would be negligible (inequality 51), and the limiting effects are obtained by adding the individual limits. If the features are of opposite sign, the limiting effect is the one with the larger magnitude. Other examples can be taken quickly from table 1 . A positive slope whose height is

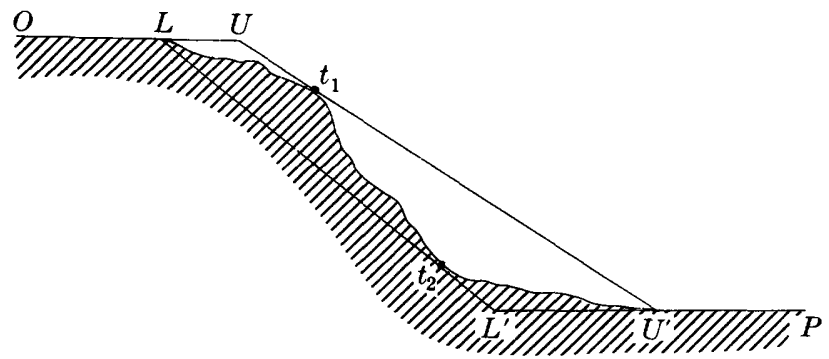

Figure 11.-Geometric bracketing of a general slope by two plane slopes. 
equal to its distance from the station cannot affect the heat flow by more than 10 percent if its maximum slope angle is $9^{\circ}$. A valley $1-\mathrm{km}$ deep with a 10-km flood plane will not increase the heat flow at its center by more than 10 percent if the walls are not steeper than $30^{\circ}$. (The interaction is negligible by inequality 51. )

When drilling holes to determine heat flow, it is desirable to select sites at which the topographic anomaly is minimized. Thus, site selection often involves making many calculations of the type just discussed. I have found it helpful to take a copy of figure 3 to the field for this purpose.

Although the bracketing described is achieved with a two-dimensional model, it can, of course, be applied to three-dimensional topographic forms.

The methods of this section often give a useful upper or lower limit to the topographic anomaly, but the condition that the bracketing slopes be everywhere above or below the real surface usually leads to bracketing intervals that are rather large. A more refined method will therefore be considered in the next section.

\section{EQUIVALENT SLOPES}

To consider the example illustrated in figure 10 in more detail we write:

$$
\begin{aligned}
& \Delta q_{a b}-\Delta q_{a}=\frac{1}{\pi} \int_{-\infty}^{+\infty} \frac{h_{a b}\left(1+e_{a b}\right)-h_{a}\left(1+e_{a}\right)}{\left(x-x_{0}\right)^{2}} d x \\
& \quad=\frac{1}{\pi} \int_{-\infty}^{+\infty} \frac{h_{b}\left(1+e_{a}\right)+h_{a b}\left(e_{a b}-e_{a}\right)}{\left(x-x_{0}\right)^{2}} d x \\
& \quad=\Delta q_{b}^{\prime}+\frac{1}{\pi} \int_{x_{1}}^{x_{2}} \frac{h_{b} e_{a}}{\left(x-x_{0}\right)^{2}} d x+\frac{1}{\pi} \int_{-\infty}^{+\infty} \frac{h_{a b} \Delta e}{\left(x-x_{0}\right)^{2}} d x,
\end{aligned}
$$

where $\Delta e(x)=e_{a b}(x)-e_{a}(x)$. (In this section and those that follow the quantity $e$ will be understood to include the quantity $\epsilon$ which applies to the special case of a plane slope.)

It was pointed out in the previous section that for $x_{0}$ not in $\left(x_{1}, x_{2}\right)$ the difference on the left side of equation 57 has the sign of $h_{b}$, which is also the sign of $\Delta q_{b}^{\prime}$. Neglecting the second and third terms of the right side of equation 57 is equivalent to approximating $\Delta q_{a b}$ as the sum of the exact solution, $\Delta q_{a}$, and the Jeffreys approximation to the perturbation $h_{b}$, that is,

$$
\Delta q_{a b} \cong \Delta q_{a}+\Delta q_{b}^{\prime} .
$$

We know that the correction term is of the right sign (if $h_{b}$ is of one sign), but it is not known that the approximation to $\Delta q_{a b}$ is any better with it than without it. 
According to equation 26, $e_{a}(x)$ represents the anomaly in the mean gradient in $h_{a}$ at $x$. Therefore, the second term on the right side of equation 57 adjusts $\Delta q_{b}^{\prime}$ for departures of the local gradient from unity at $h_{b}$.

Thus the approximation:

$$
\Delta q_{a b} \cong \Delta q_{a}+\Delta q_{b}^{\prime}+\frac{1}{\pi} \int_{x_{1}}^{x_{2}} \frac{h_{b} e_{a}}{\left(x-x_{0}\right)^{2}} d x
$$

is a refined Jeffreys-type representation of the perturbation in which the effect on reference-plane temperature is adjusted for local conditions of heat flow through $h_{b}$.

It is seen that positive $e_{a}$ (high heat flow through $h_{b}$ ) increases the magnitude of the correction, and negative $e_{a}$ reduces it as might be expected. In this sense, $e_{a}$ can be viewed as a weighting function.

The $\Delta e(x)$ in the third term of equation 57 represents the reaction of the mean gradient throughout the topography occasioned by adding the perturbation $h_{b}$ to $h_{a}$. If $h_{a}$ is positive and $h_{b}$ negative, $\Delta e$ will tend to be positive in $\left(x_{1}, x_{2}\right)$ because of the downward crowding of the isotherms. It will, however, tend to be negative for $x$ not in $\left(x_{1}, x_{2}\right)$ for reasons discussed in the previous section. For $h_{b}$ positive the opposite holds true. Unlike the first and second terms, which are integrated only over the region of the perturbation, the third term is integrated over an infinite domain. It is to some extent selfcanceling, as it tends to be of opposite sign to $\Delta q_{b}^{\prime}$ over the range $x_{1}<x<x_{2}$ and of the same sign in $x<x_{1}, x>x_{2}$.

It is clear that the errors arising from approximating $h_{a b}$ with a plane slope $h_{a}$ and correction terms tend to grow with the magnitude of $h_{b}$. A better approximation might therefore be obtained by selecting $h_{a}$ in such a way that $h_{b}$ has parts of both signs so that its magnitude is reduced, and the correction terms tend to cancel.

In order to consider rather general topographic configurations, and still restrict the discussion to slopelike forms, we shall define a "slope form" as two horizontal halfplanes joined by a general (two-dimensional) surface whose highest point is the intersection with the upper halfplane (the brink) and whose lowest point is the intersection with the lower halfplane (the toe). (Where no ambiguity will result we shall refer to this figure simply as a "slope.") The width of the sloping portion of a slope form is finite; hence it does not include the monocline of Jaeger and Sass (1963). Consider the slope form $h_{a b}>0$ whose toe is at $\xi_{1}$ and brink at $\xi_{2}$ (fig. 12). To approximate the heat flow at $x_{0}$ in front of the toe we consider $h_{a b}$ as the sum of the plane slope of the same height, $h_{a}$, and the element, $h_{b}$, with positive and negative portions. 

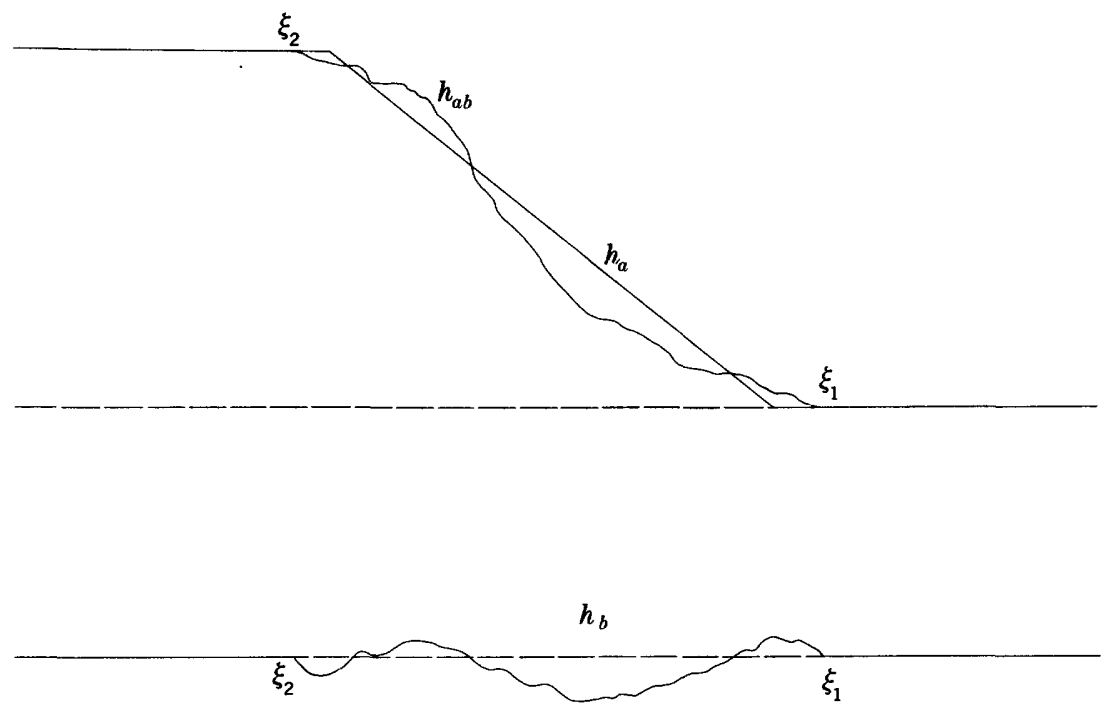

Figure 12.-Notation for the discussion of slope forms.

If $h_{a}$ is chosen in such a way that:

$$
\int \frac{h_{a b}-h_{a}}{\left(x-x_{0}\right)^{2}} d x=0
$$

then $\Delta q_{b}^{\prime}\left(x_{0}\right)$ vanishes, and hence:

$$
\Delta q_{a b}^{\prime}\left(x_{0}\right)=\Delta q_{a}^{\prime}\left(x_{0}\right) .
$$

Thus any two slopes $h_{a b}$ and $h_{a}$ that satisfy 60 must have the same Jeffreys approximation at $x_{0}$. They will be referred to as "equivalent slopes at $x_{0}$ ". If $h_{a}$ is chosen to be equivalent to $h_{a b}$ at $x_{0}$, equation 57 becomes:

$$
\Delta q_{a b}\left(x_{0}\right)=\Delta q_{a}\left(x_{0}\right)+\frac{1}{\pi} \int \frac{h_{b} e_{a}}{\left(x-x_{0}\right)^{2}} d x+\frac{1}{\pi} \int \frac{h_{a b} \Delta e}{\left(x-x_{0}\right)^{2}} d x .
$$

This choice automatically assures that the first-order contributions of irregular topographic features are accounted for, so that after weighting their height by the inverse square of their distance from the station, their sum is zero. It also assures a symmetry that justifies the interpretation of $e_{a}$ as a weighting function in the second-order correction term, and it leads to canceling effects both in and out of $\left(\xi_{1}, \xi_{2}\right)$ in the third-order term.

It is clear that any two slopes equivalent to a third slope at $x_{0}$ are equivalent to each other there. For any slope $h_{a b}$ there is a family of 
equivalent plane slopes at any point $x_{0}$ not on the slopes. This can be seen by noting that given $\hat{h}_{a}$ equivalent to $h_{a b}$ (fig. 13) another slope, $h_{a}^{*}$, equivalent to $h_{a}$ can be drawn by increasing the slope angle and distance from the station simultaneously in such a way as to keep $\Delta q_{a}^{\prime}$ constant. Thus, the flatter the equivalent plane slope, the closer it extends toward the station. The equivalent cliff is the member of the family farthest from the station.

For any slope form it will be possible to select plane slopes equivalent at $x_{0}$ in sucl a way that $h_{b}$ has one positive and one negative section, as long as $x_{0}$ lies a finite distance beyond the toe or behind the brink. We shall denote the section near the toe by $h_{b 1}$ and the other by $h_{b 2}$.
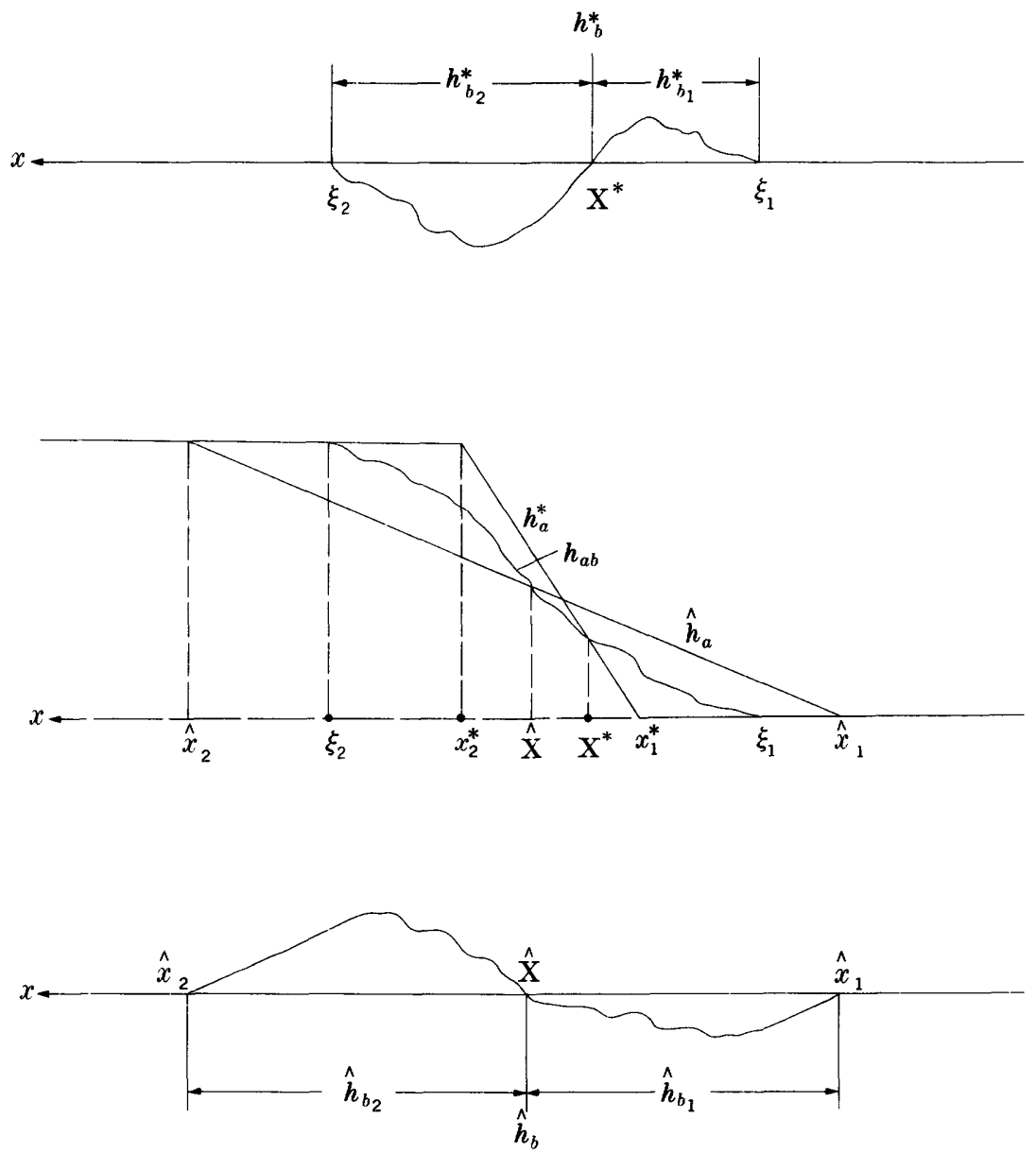

Figure 13.--Notation for the discussion of equivalent plane slopes $\left(h_{a}^{*}\right.$ and $\left.\hat{h}_{a}\right)$. 
Those plane equivalent slopes that are steeper than $h_{a b}$, in the sense that they make $h_{b_{1}}$ positive, will be denoted by $h_{a}^{*}$. The flatter ones (which make $h_{b_{1}}$ negative) will be denoted by $\hat{h}_{a}$ (fig. 13). The heatflow anomalies produced at $x_{0}$ by $h_{a}^{*}$ and $\hat{h}_{a}$ will be denoted $\Delta q_{a}^{*}\left(x_{0}\right)$ and $\Delta \hat{q}_{a}\left(x_{0}\right)$ respectively. The coordinates of the toe and brink of $h_{a b}$ will be denoted respectively $\xi_{1}$ and $\xi_{2}$, and those of $\hat{h}_{a}$ and $h_{a}^{*}$ by $\hat{x}_{1}, \hat{x}_{2}$ and $x_{1}^{*}, x_{2}^{*}$. The coordinate of the intersection of $h_{a b}$ with the sloping parts of $\hat{h}_{a}$ or $h_{a}^{*}$ are denoted respectively by $\hat{X}$ or $X^{*}$. Other quantities related to these slopes such as $e_{a}$ and $h_{b}$ will also be distinguished by * or ${ }^{\wedge}$.

Replacing $h_{a}$ by $\hat{h}_{a}$ in equation 57 yields:

$\Delta q_{a b}\left(x_{0}\right)-\Delta \hat{g}_{a}\left(x_{0}\right)=\frac{1}{\pi} \int_{\hat{x}_{1}}^{\hat{x}} \frac{\hat{h}_{b_{1}} \hat{e}_{a}}{\left(x-x_{0}\right)^{2}} d x+\frac{1}{\pi} \int_{\hat{X}}^{\hat{x}_{2}} \frac{\hat{h}_{b_{2}} \hat{e}_{a}}{\left(x-x_{0}\right)^{2}} d x+\frac{1}{\pi} \int_{\xi_{1}}^{\infty} \frac{h_{a b} \hat{\Delta} e}{\left(x-x_{0}\right)^{2}} d x$.

However, from equation 60 we know that:

$$
\int_{\hat{x}_{1}}^{\hat{X}} \frac{\hat{h}_{b_{1}}}{\left(x-x_{0}\right)^{2}} d x=-\int_{\hat{X}}^{\hat{x}_{2}} \frac{\hat{h}_{b_{2}}}{\left(x-x_{0}\right)^{2}} d x .
$$

Inasmuch as $\hat{e}_{a}$ is a decreasing function of distance from the toe $\left(x-\hat{x}_{1}\right)$ in $\left(\hat{x}_{1}, \hat{x}_{2}\right)$ the sum of the two second-order integrals assumes the sign of $h_{b_{1}}$, which, in this case, is negative.

As long as the second-order terms dominate the third-order integral (which represents interactions that generally tend to cancel) we have the inequality:

$$
\Delta \hat{q}_{a}\left(x_{0}\right)>\Delta q_{a b}\left(x_{0}\right)
$$

Replacing $h_{a}$ by $h_{a}^{*}$ in equation 57 and rearranging the integrands we obtain:

$\Delta q_{a b}\left(x_{0}\right)-\Delta q_{\alpha}^{*}\left(x_{0}\right)=\frac{1}{\pi} \int_{\xi_{1}}^{X *} \frac{h_{b_{1}}^{*} e_{a b}}{\left(x-x_{0}\right)^{2}} d x+\frac{1}{\pi} \int_{X *}^{\xi_{2}} \frac{h_{b_{2}}^{*} e_{a b}}{\left(x-x_{0}\right)^{2}} d x+\frac{1}{\pi} \int_{x_{1}^{*}}^{\infty} \frac{h_{a}^{*} \Delta e^{*}}{\left(x-x_{0}\right)^{2}} d x$.

The changed form of the integrands is necessary because $e_{a}^{*}$ is not defined over the entire domain of $h_{b}^{*}$, but $e_{a b}$ is. If $e_{a b}$ is generally larger in the lower part of the slope (below $X^{*}$ ) than in the upper part, the second-order integrals will again take the sign of $h_{b_{1}}$, now positive. Assuming again that the third-order effects are dominated we have:

$$
\Delta q_{a},\left(x_{0}\right)>\Delta q_{a}^{*}\left(x_{0}\right) .
$$


In developing inequalities 65 and 67 we have used the example of $h_{a b}$ positive-the point $x_{0}$ was beyond the toe. If $x_{0}$ lies behind the brink, $h_{a b}$ and $h_{a}$ are negative and occupy all or part of the region $x_{2}>x>-\infty$. For this case the equation corresponding to 63 is:

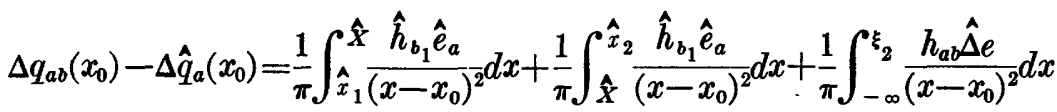

and only the integrand in the third integral undergoes a change of sign. Thus the arguments leading to inequalities 65 and 67 apply whether $h_{a b}$ is positive or negative.

There is, however, one difference between the two examples worth noting. From equation 64 it is seen that:

$$
\left|\int h_{b_{1}} d x\right|<\left|\int h_{b_{2}} d x\right|, h_{a b}>0,
$$

where the integrations are carried out over the domains in which the integrands are defined. For positive $h_{a b}$, the closer $x_{0}$ lies to the toe of the slope, the stronger inequality 69 becomes, as a very small $h_{b_{1}}$ will balance a very large $h_{\delta_{2}}$ when they are weighted by the inverse square of their distance from the station. Inequalities 65 and 67 arise from the difference in the mean values of the weighting function, $e_{a}$ (or $e_{a b}$ ), in the two integrals. Inasmuch as $\frac{d e}{d x}$ is generally greatest near the toe, the difference between the mean value of $e$ in the two integrals will be greater as the width of $h_{b_{1}}$ becomes smaller. Thus the closer $x_{0}$ comes to the toe, the stronger inequalities 65 and 67 tend to become.

For negative $h_{a b}, h_{b_{1}}$ and $h_{b_{2}}$ are multiplied by the inverse square of their distance from the brink in equation 64 . Thus:

$$
\left|\int h_{b_{1}} d x\right|>\left|\int h_{b_{2}} d x\right|, h_{a b}<0
$$

and for $x_{0}$ very near the brink a very small $h_{b_{2}}$ will balance a very large $h_{b_{1}}$; as $x_{0}$ approaches the brink so does $X$. However, near the brink the weighting functions $e_{a}$ and $e_{a b}$ are generally undergoing their least change with $x$. Thus the closer $x_{0}$ comes to the brink, the weaker inequalities 65 and 67 tend to become.

To summarize we combine inequalities 65 and 67 :

$$
\Delta q_{a}^{*}\left(x_{0}\right)<\Delta q_{a b}\left(x_{0}\right)<\Delta \hat{q}_{a}\left(x_{0}\right) .
$$


The mathematical conditions on $h_{a b}$ and $x_{0}$ sufficient for the validity of inequality 71 are:

1. That $h_{a b}$ be a slope form.

2. That $e_{a b}$ generally be greater below the intersection $\left(X^{*}\right)$ of $h_{a b}$ with $h_{a}^{*}$ than above it.

3. That the second-order terms dominate the higher order interaction effects.

The condition that $h_{a b}$ be a slope form assures that flatter equivalent plane slopes $\hat{h}_{a}$ and steeper ones $h_{a}^{*}$ can be drawn for any $x_{0}$ not on the sloping portion. The second condition is less explicit than we might like, but it is difficult to imagine cases in which it would be violated. This can be made clearer by rewriting equation 66 :

$$
\begin{aligned}
\Delta q_{a b}\left(x_{0}\right)-\Delta q_{a}^{*}\left(x_{0}\right)=\frac{1}{\pi} \int_{i_{1}}^{x_{1}^{*}} \frac{h_{b_{1}} e_{a b}}{\left(x-x_{0}\right)^{2}} d x & +\frac{1}{\pi} \int_{x_{1}^{*}}^{X *} \frac{h_{b_{1}} e_{a}^{*}}{\left(x-x_{0}\right)^{2}} d x \\
& \quad+\frac{1}{\pi} \int_{X^{*}}^{\infty} \frac{h_{b_{2}} e_{a}^{*}}{\left(x-x_{0}\right)^{2}} d x+\frac{1}{\pi} \int_{x_{1}^{*}}^{\infty} \frac{h_{a b} \Delta e^{*}}{\left(x-x_{0}\right)^{2}} d x .
\end{aligned}
$$

Because $h_{a}^{*}$ is equivalent to $h_{a b}$, we know the sum of the first two integrals would be the negative of the third integral if the $e$ 's were unity. In this form the weighting function is $e_{a}^{*}$ (a strongly decreasing function of $x$ ) in $x_{a}^{*}<x<\infty$, and $e_{a b}$ in the region near the toe, $x_{1}^{*}>x>\xi_{1}$, where it would generally be positive. Any slope $h_{a b}$ for which the first two integrals did not dominate the third must be very unusual. For example it might be steep at the top with very thin, high ridges (resembling cooling fins) near the toe. It could hardly be important in terrestrial heat-flow applications.

Violations of the last condition are most likely to occur at points very close to the brink where the heat flow is very close to zero, and small effects of higher order can dominate.

\section{TFSTS OF INEQUALITY 71}

Unfortunately, the only available exact solution for a slope of finite width (a slope form) is that derived for the plane slope in the second section. Therefore, to test the inequality we shall represent $h_{a b}$ by the plane slope of angle $\beta$, and approximate its effect at any point $x_{0}$ by equivalent plane slopes $\hat{h}_{a}$ of angle $\hat{\beta}$ and $h_{a}^{*}$ of angle $\beta^{*}$ where:

$$
\boldsymbol{\beta}^{*}>\boldsymbol{\beta}>\hat{\boldsymbol{\beta}} .
$$

Results for two examples $\left(\beta=30^{\circ}, \beta^{*}=45^{\circ}, \hat{\beta}=15^{\circ}\right.$; and $\beta=15^{\circ}$, $\beta^{*}=22 \frac{1}{2} 2^{\circ}, \hat{\beta}=9^{\circ}$ ) are presented in figure 14 . 


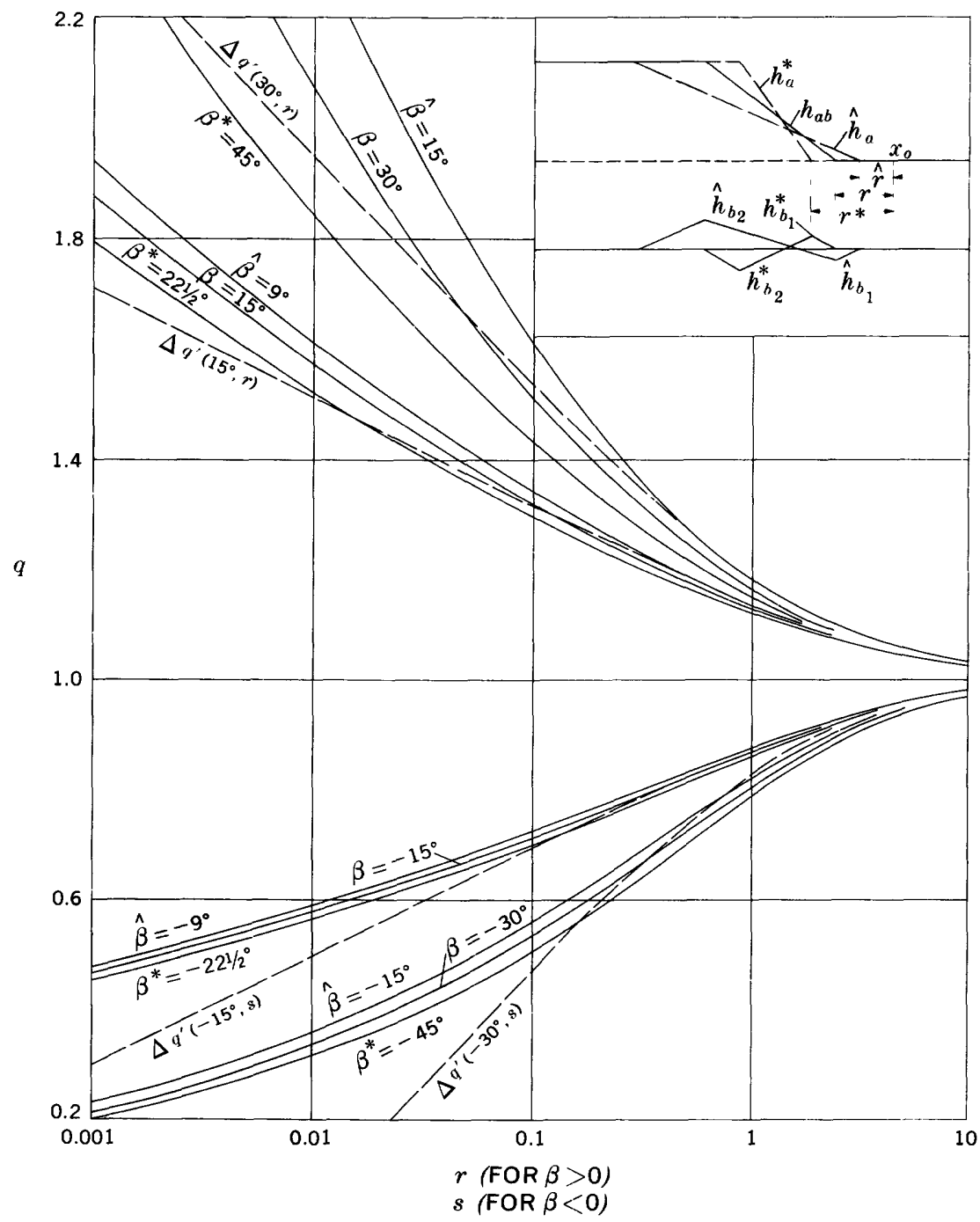

FigURe 14.-Comparison of the anomaly caused by a plane slope of angle $\beta$ with the anomaly from bracketing equivalent plane slopes of angle $\hat{\beta}$ and $\beta^{*}$. Jeffreys approximation is shown as dashed line.

In this example inequality 71 holds over the range of four orders of magnitude of $r$ and $s$. It is seen that the bracketing interval for $s$ (points behind the brink) is smaller than the corresponding one for $r$, and that it tends to decrease for very small $s$ whereas it increases for very small $r$. Inasmuch as a weakening of inequality 71 is equivalent to a decrease in the bracketing interval, these results are consistent with the foregoing discussion. 
To the extent that inequality 71 applies, it should generally follow that in any family of plane slopes of equal height which yield the same Jeffreys approximation at points on the horizontal surfaces the heat flow should decrease with increasing slope angle. This relation is tested numerically in tables 4 and 5 where each pair of columns represents members of a family of plane equivalent slopes whose Jeffreys approximation is the value of $q^{\prime}$ given at the top. The column headed $r$ (or $s$ ) gives the distance of the toe (or brink) from the station, and the column headed $q$ gives the exact value of the heat flow there. It is seen that the relation is satisfied except for points very close to the brink for the steepest angles (see the two entries with asterisks, case $q^{\prime}=-1.00$, table 5). Fortunately, for such points a reliable lower limit is usually obtained easily by geometric bracketing. It is generally found to be close to the values given by inequatity 71 in any case.

TABLE 4.-Comparison of exact solutions for families of positive equivalent plane slopes

[Powers of 10 are denoted by their exponents in parentheses]

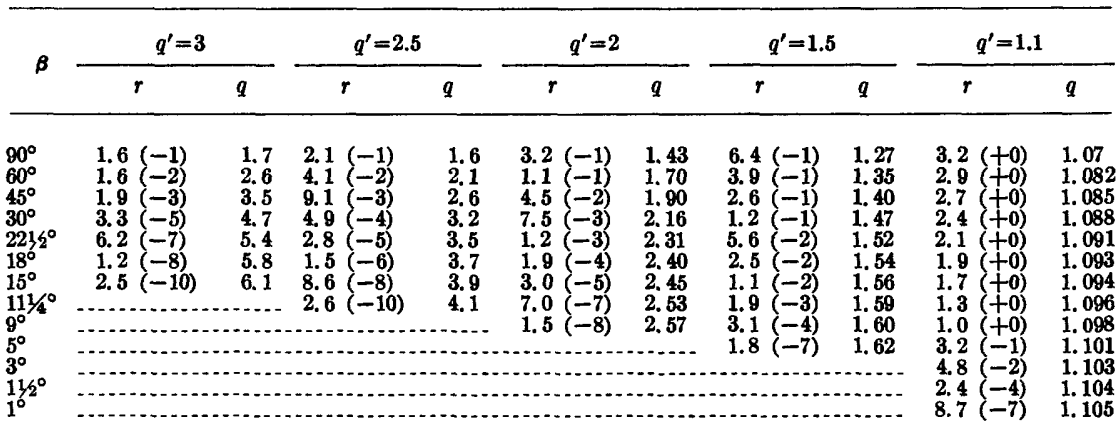

TABLE 5.-Comparison of exact solutions for families of negative equivallent plane slopes

[Powers of 10 are denoted by their exponents in parentheses]

\begin{tabular}{|c|c|c|c|c|c|c|c|c|c|c|}
\hline \multirow{2}{*}{$\boldsymbol{\beta}$} & \multicolumn{2}{|c|}{$q^{\prime}=-1.00$} & \multicolumn{2}{|c|}{$q^{\prime}=-0.50$} & \multicolumn{2}{|c|}{$q^{\prime}=0.00$} & \multicolumn{2}{|c|}{$q^{\prime}=+0.50$} & \multicolumn{2}{|c|}{$q^{\prime}=+0.90$} \\
\hline & $\boldsymbol{s}$ & $q$ & 8 & $q$ & $\boldsymbol{8}$ & $q$ & 8 & $q$ & $\boldsymbol{s}$ & $q$ \\
\hline $\begin{array}{l}90^{\circ} \\
60^{\circ} \\
45^{\circ} \\
30^{\circ} \\
2212^{\circ} \\
18^{\circ} \\
15^{\circ} \\
114^{\circ} \\
9^{\circ} \\
5^{\circ} \\
3^{\circ} \\
112^{\circ} \\
1^{\circ}\end{array}$ & \begin{tabular}{l}
$1.6(-1)$ \\
$1.6(-2)$ \\
$1.9(-3)$ \\
$3.3(-5)$ \\
$6.2(-7)$ \\
$1.2(-8)$ \\
$2.4(-10)$ \\
\hdashline
\end{tabular} & $\begin{array}{l}0.124^{*} \\
.105^{*} \\
.103 \\
.107 \\
.112 \\
.115 \\
.118 \\
. . .\end{array}$ & $\begin{array}{l}2.1(-1) \\
4.1(-2) \\
9.1(-3) \\
4.9(-4) \\
2.8(-5) \\
1.5(-6) \\
8.6(-8) \\
2.6(-10)\end{array}$ & $\begin{array}{r}0.164 \\
.168 \\
.175 \\
.185 \\
.192 \\
.197 \\
.200 \\
.206 \\
. .-. .\end{array}$ & $\begin{array}{l}3.2(-1) \\
1.1(-1) \\
4.5(-2) \\
7.5(-3) \\
1.2(-3) \\
1.9(-4) \\
3.0(-5) \\
7.0(-7) \\
1.5(-8)\end{array}$ & $\begin{array}{r}0.240 \\
.278 \\
.298 \\
.319 \\
.330 \\
.336 \\
.341 \\
.347 \\
.351 \\
. .1 .\end{array}$ & $\begin{array}{l}6.4(-1) \\
3.9(-1) \\
2.6(-1) \\
1.2(-1) \\
5.6(-2) \\
2.5(-2) \\
1.1(-2) \\
1.9(-3) \\
3.1(-4) \\
2.0(-5) \\
1.8(-7)\end{array}$ & $\begin{array}{l}0.434 \\
.496 \\
.525 \\
.553 \\
.567 \\
.576 \\
.581 \\
.588 \\
.591 \\
.595 \\
.598\end{array}$ & $\begin{array}{l}3.2(-0) \\
2.9(-0) \\
2.7(-0) \\
2.4(-0) \\
2.1(-0) \\
1.9(-0) \\
1.7(-0) \\
1.3(-0) \\
1.0(-0) \\
3.2(-1) \\
4.8(-2) \\
2.4(-4) \\
8.7(-7)\end{array}$ & $\begin{array}{r}.8725 \\
.8807 \\
.8848 \\
.8895 \\
.8924 \\
.8943 \\
.8958 \\
.8978 \\
.8991 \\
.9018 \\
.9031 \\
.9040 \\
.9043\end{array}$ \\
\hline
\end{tabular}




\section{SLOPES CONCAVE AT THE TOF OR CONVEX AT THE BRINK}

To investigate some of the implications of inequality 71 it is convenient to represent $D$ as a function of $\beta$ and $\Delta q^{\prime}$ (figs. 15 and 16), where $D$ is the quantity that must be added to $\Delta q^{\prime}$ to obtain $\Delta q$ for a plane slope of angle $\beta$.

$$
D\left(\beta, \Delta q^{\prime}\right)=\Delta q\left(\beta, \Delta q^{\prime}\right)-\Delta q^{\prime} .
$$

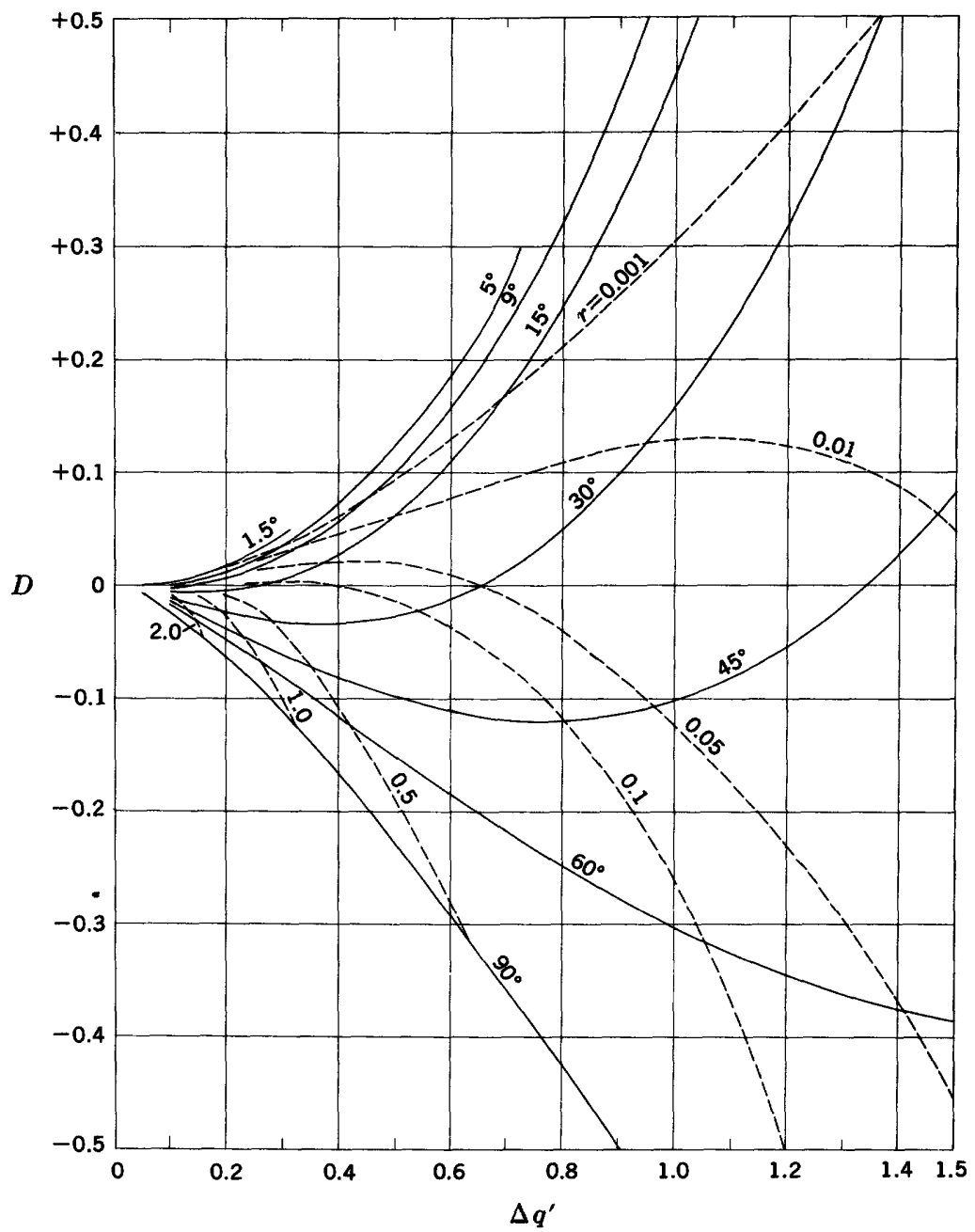

FIGURE 15.-Error in the Jeffreys approximation $(D)$ as a function of the Jeffreys approximation $\left(\Delta q^{\prime}\right)$ for constant positive slope angle, $\beta$ (solid curves), or constant distance from the toe, $r$ (dashed curves). 


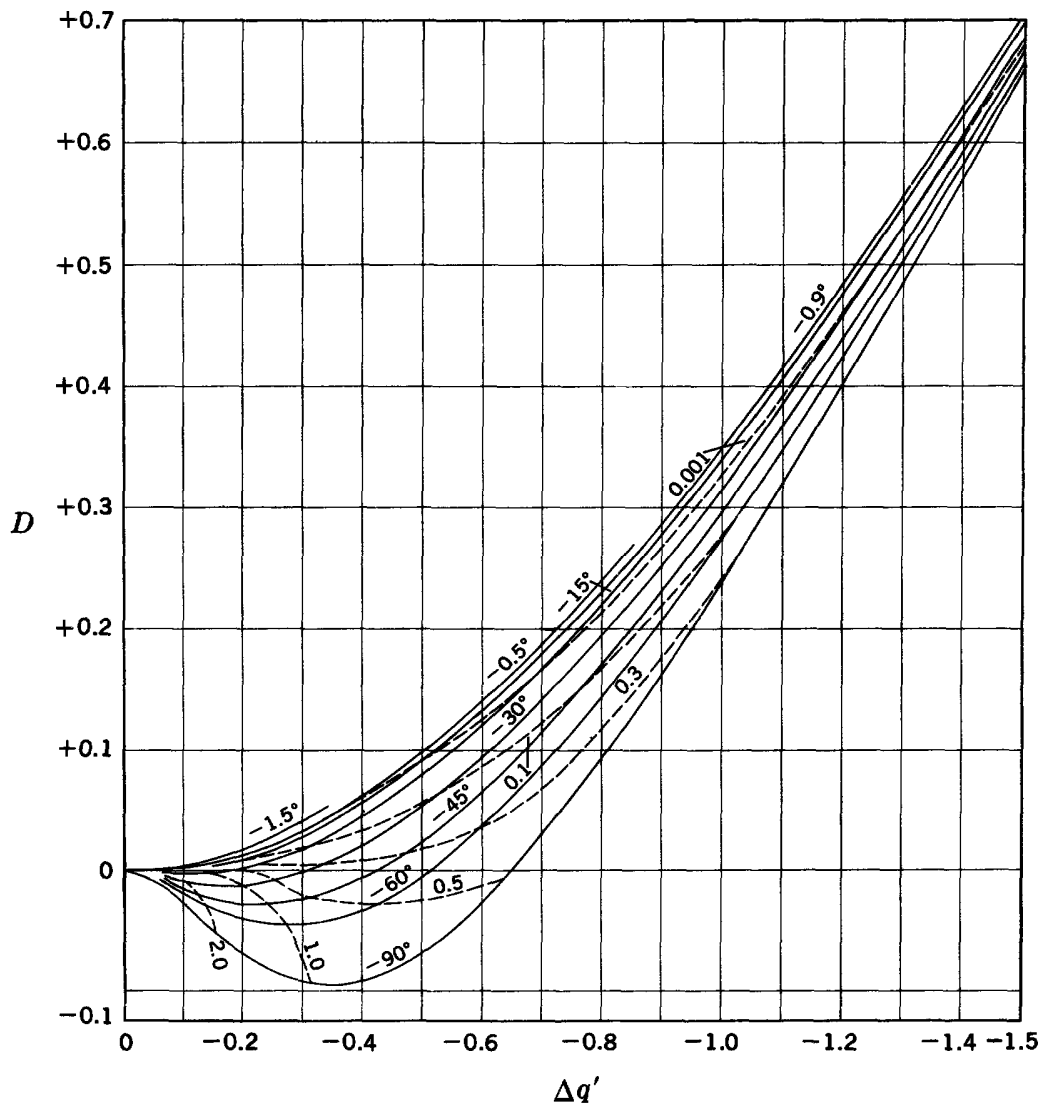

Figure 16.-Error in the Jeffreys approximation $(D)$ as a function of the Jeffreys approximation $\left(\Delta q^{\prime}\right)$ for constant negative slope angle, $\beta$ (solid curves), or constant distance from the brink, $s$ (dashed curves).

We have just seen that generally:

$$
D\left(\beta_{1}, \Delta q^{\prime}\right)>D\left(\beta_{2}, \Delta q^{\prime}\right), \beta_{1}<\beta_{2},
$$

that is, smaller angles yield (algebraically) larger $D$ 's for the same $\Delta q^{\prime}$. It is seen that this is true over the range of figures 15 and 16 . In this notation, inequality 71 becomes:

$$
D^{*}\left(\beta^{*}, \Delta q^{\prime}\left(x_{0}\right)\right)<D\left(\Delta q^{\prime}\left(x_{0}\right)\right)<\hat{D}\left(\hat{\beta, \Delta} q^{\prime}\left(x_{0}\right)\right) .
$$

The bracketing interval in inequality 75 (or inequality 71) can be read directly from figures 15 and 16 for any $\Delta q^{\prime}$ for selected angles, $\beta^{*}$ and $\hat{\beta}$, of the bracketing plane slopes. (For example, if $\Delta q^{\prime}=+0.5$, 
$\beta^{*}=60^{\circ}, \hat{\beta}=45^{\circ}$, then the interval is 0.05 .) The limiting values of $\Delta q$ in equality 71 can be determined by adding the limiting values of $D$ from figures 15 or 16 to the abscissa, $\Delta q^{\prime}$. (In the example $D^{*}=-0.15, \hat{\mathrm{D}}=-0.10$ and in equality 71 gives $0.35<\Delta q<0.40$.)

From inequality 74 or from figures $14-16$, it is clear that smaller bracketing intervals are obtained by selecting smaller values of $\beta^{*}$ and larger values of $\hat{\beta}$. The smallest value of $\beta^{*}$ is associated with the plane slope, $h_{a}^{*}\left(x_{0}\right)$, that is closest to the station. The largest value of $\hat{\beta}$ is associated with the plane slope, $\hat{h}_{a}\left(x_{0}\right)$, that is farthest from the station. At present we are chiefly concerned with the upper limit and $\hat{h}_{a}$. For positive relief $x_{1}$ cannot be farther from the station than $\xi_{1}$ (fig. 13) because of the condition $\hat{h}_{b_{1}}<0$. Similarly for negative relief, $x_{2}$ cannot be farther from the station than $\xi_{2}$ by the condition $h_{b_{2}}>0$. Hence the best $h_{a}$, that is, the one that gives the lowest upper limit in inequality 71 , would be the one that passed through the toe, $\xi_{1}$, for a positive slope, or through the brink, $\xi_{2}$, for a negative slope. However, not all slopes contain such plane slopes in their family of permissible $h_{a}$ 's. For example, on a long positive slope which steepens very near the toe it is generally not possible to draw an equivalent plane slope through the toe which lies above the slope at the toe. In such an example the steepest $h_{a}$ has its toe, $x_{1}$, closer to the station than $\xi_{1}$, and $\beta$ is reduced correspondingly. This in turn increases the upper limit on $q$ in inequality 71 as is seen clearly in figure 15. This situation corresponds to the physical expectation that for two positive slopes with the same $\Delta q^{\prime}\left(x_{0}\right)$, the one with the greater concentration of volume near the toe will generally yield the greater $\Delta q\left(x_{0}\right)$ because this volume lies in the region of greatest gradients. Similarly, it is not generally possible to draw $h_{a}$ through the brink of slopes which steepen very close to the brink. Fortunately, most slopes in the earth's surface tend to decrease in inclination near the toe and brink. We shall call a positive slope "concave at the toe" if it is possible to draw $\hat{h}_{a}\left(x_{0}\right)$ through the toe, $\xi_{1}$. A negative slope will be called "convex at the brink" if $\hat{h}_{a}\left(x_{0}\right)$ can be drawn through the brink. Most natural slopes can be represented by models in these categories.

In figure 15 any vertical coordinate line can be viewed as representing a family of equivalent plane slopes, $\Delta q^{\prime}(\beta, r)=$ constant. The members of this family can be identified with reference to the coordinate curves of $\beta$ and $r$. Thus, in the family of equivalent slopes for which $\Delta q^{\prime}=+0.35$, that member which yields $D=-0.07$ is the $45^{\circ}$ slope at a distance $r=0.5$ from the station. Any point vertically below the intersection $r=0.5, \beta=45^{\circ}$, corresponds to a steeper equivalent slope farther from the station; points above the intersection correspond 
to equivalent slopes for which $\beta<45^{\circ}, r<0.5$. If we are given a positive slope, concave at the toe, whose height is twice its distance from the station $(r=0.5)$ and for which $\Delta q^{\prime}\left(x_{0}\right)=0.35$, we know from the previous discussion that $45^{\circ}$ must be the best choice of $\hat{\beta}$. Hence for any such slope $\hat{D}=-0.07$ and $\Delta \hat{q}$ is 0.28 . We can always choose $\beta^{*}=90^{\circ}$ which yields (fig. 15) $D^{*}=-0.14$ and $\Delta q^{*}\left(x_{0}\right)=0.21$. Any other positive slope concave at the toe for which $\Delta q^{\prime}=0.35$ which was farther from the station $(r>0.5)$ would have a larger $\hat{\beta}$ and hence its upper limit $\hat{D}$ would correspond to an ordinate between -0.07 and -0.14 . More generally, any positive slope concave at the toe, whose height does not exceed twice its distance from the station, is represented by points in figure 15 between the curves $r=0.5$ and $\beta=90^{\circ}$. Inasmuch as the maximum difference in the ordinate between these curves is 0.07 , any of these slopes can be replaced by the equivalent cliff with an error not exceeding 7 percent. From the curve $r=1$, it is seen that any positive slope, concave at the toe, whose height does not exceed its distance from the station can be represented by the equivalent cliff with errors less than 3 percent; if the height is less than 50 percent of the distance from the station (fig. 15, r $>2$ ) the error is less than $1 \frac{1}{2}$ percent. The corresponding results for negative slopes, convex at the brink, are 4 percent for $s>1$ and $11 / 2$ percent for $s>2$ (fig. 16).

\section{SOME CONDITIONS FOR VALIDITY OF THE JEFFREYS APPROXIMATION}

From many of the numerical results presented (see fig. 2) the Jeffreys approximation often gives a satisfactory value for the topographic correction at the surface. However, it is extremely difficult to establish general conditions under which the approximation is valid and without them it cannot be applied with confidence. Consideration of the equivalent slopes provides some insight into this elusory problem.

It is seen from figure 15 that in the region between the curves $\beta=30^{\circ}$ and $r=0.05$ the magnitude of $D$ does not exceed about 0.03 . Therefore, any positive slope, concave at the toe, whose height does not exceed 20 times its distance from the station and whose maximum slope angle does not exceed $30^{\circ}$, can be represented by the Jeffreys approximation with errors not exceeding about 3 percent. (The actual condition on the slope angle is somewhat more general, namely that there exists a $\beta^{*} \leq 30^{\circ}$.) This description applies to a broad class of slopes of general interest, with positive anomalies ranging up to 65 percent. Similarly, the error would not exceed 13 percent for positive slopes, concave at the toe, even if their height were 100 times their distance from the station as long as the maximum slope angle (actually minimum $\beta^{*}$ ) 
were less than $45^{\circ}$ (fig. 15). If a scarp occurred near the toe (so that the slope were not concave there), and it was necessary to select an $\hat{h}_{a}$ that extended to within $10^{-3}$ slope heights of the station, the linear approximation might contain substantial errors if $\Delta q^{\prime}$ were large (fig. 15).

From figure 16 it is seen that negative slopes, convex at the brink, can be approximated by the Jeffreys method to within 3 percent if their height does not exceed about three times their distance from the station and the maximum slope angle does not exceed about $45^{\circ}$. Virtually any slope, positive or negative, irrespective of slope at the toe or brink can be represented by the Jeffreys approximation within a few percent as long as $\Delta q^{\prime}$ does not exceed 0.15 and $\beta^{*}<60^{\circ}$.

In general, it is seen (fig. 16) that for negative slopes the Jeffreys approximation is poor when $\Delta q^{\prime}$ is large because the approximation becomes negatively infinite at the brink, where the actual heat flow approaches zero.

It should be noted that although the Jeffreys approximation might apply to the independent effects of slopes on either side of a station, it does not apply generally when the two coexist. Their interaction must be considered, as it must for any other slopes. It is also seen that small $\Delta q^{\prime}$ is not a sufficient condition for validity of the Jeffreys approximation unless coexisting slopes are of the same sign.

\section{BRACKETING WITH RQUIVALENT PLANE SLOPES: NUMFRICAL RESULTS}

Inequality 71 can be applied to obtain rapid estimates of heat-flow anomalies on horizontal surfaces near irregular slopes. The calculation would normally be performed in three steps:

1. Compute $\Delta q_{a b}^{\prime}\left(x_{0}\right)$ for the given slope $h_{a b}$ at the station $\left(x_{0}\right)$ (pl. 2);

2. Select a steeper equivalent plane slope $h_{a}^{*}$ and a flatter one $\hat{h}_{a}$ (pl. 2);

3. Determine the limiting values of the anomaly $\Delta q_{a}^{*}$ and $\Delta \hat{q}_{a}$ (pl. 1).

These calculations can usually be facilitated by the use of plate 2, which provides a rapid means of estimating $\Delta q^{\prime}, \beta$, or distance of the station from the slope $(r$ or $s)$ if any two are known. The curves below and to the left of the line $\beta=90^{\circ}$ are used with the lower abscissal scale when working with distances from the toe of positive relief $(r)$ or from the brink of negative relief $(s)$. It is sometimes more convenient to consider distances from the brink of positive slopes $(r+\cot \beta)$ or from the toe of negative slopes $(s+\cot \beta)$; in this case the upper righthand curves and the upper abscissal scale of plate 2 apply. For many general slopes $\Delta q^{\prime}$ can be estimated rapidly (step 1) by approximating 
them as the sum of plane slopes whose contributions are determined from plate 2. Special care should be taken in approximating topography close to the station.

It will often be possible to select values for $\beta^{*}$ and $\hat{\beta}$ by inspection, of the slope, and to obtain limiting values of the anomaly (step 3 ) directly from figures 15 and 16 . However, when seeking the smallest bracketing intervals, it will usually be necessary to determine the coordinates of the toes of the bracketing slopes $\left(h_{a}^{*}\right.$ and $\left.\hat{h}_{a}\right)$ to see if these slopes satisfy the requirement that they cross $h_{a b}$ at only one point. It might be necessary to test this by drawing them on the topographic cross section.

A few simple numerical examples follow:

Example 1.-Suppose the upper half of $h_{a b}$ is a vertical cliff and the lower half is a $45^{\circ}$ slope and that the heat-flow station, $x_{0}$, lies one half of a slope height beyond the toe; that is, $r=0.5$ (fig. 17A).

Step 1: To compute $\Delta q_{a b}^{\prime}\left(x_{0}\right)$ we break $h_{a b}$ into two plane slopes and add their individual contributions (pl. 2):

$$
\Delta q_{a b}^{\prime}(r=0.5)=\Delta q^{\prime}\left(90^{\circ}, r=2\right)+\Delta q^{\prime}\left(45^{\circ}, r=1\right)=0.16+0.22=0.38 \text {. }
$$

Step 2: The ordinate line $\Delta q^{\prime}=0.38$ of plate 2 identifies the coordinates $(\beta, r)$ of the family of plane slopes equivalent to $h_{a b}$ at $x_{0}$. We must select a slope flatter $\left(\hat{h}_{a}\right)$ than $h_{a b}$ and a slope steeper $\left(h_{a}^{*}\right)$, and each must intersect the sloping part of $h_{a b}$ only once. We have seen that the best choices are the most distant flatter slope and the closest steeper slope. In this example the best choice for the flatter slope, $\hat{h}_{a}$, is the one that passes through the toe of $h_{a b}$ and hence has $\hat{r}=0.5$. From plate 2 we find for $\hat{r}=0.5, \Delta q^{\prime}=0.38$ that $\hat{\beta}=51^{\circ}$. The best choice for the steeper slope, $h_{a}^{*}$, in this example is the one through the brink. To find this slope the upper scale in plate 2 is used. We set $r^{*}+$ $\cot ^{*}=1$, the distance between $x_{0}$ and the brink of $h_{a b}$, and find $\beta^{*}=70^{\circ} . \quad r^{*}=1-\cot \beta^{*}=0.64$.

Step 3: From plate 1:

hence:

$$
\begin{gathered}
\Delta \hat{q}_{a}\left(51^{\circ}, 0.5\right)=0.29, \\
\Delta q_{a}^{*}\left(70^{\circ}, 0.64\right)=0.26,
\end{gathered}
$$

$$
1.26<q_{a b}(r=0.5)<1.29 \text {. }
$$

In the present example we can obtain a result almost as good as inequality 76 directly from figure 15 . From inspection of the slope (fig. $17 \mathrm{~A}$ ) it is clear that we may choose:

$$
\begin{gathered}
\hat{\beta}=45^{\circ}, \\
\beta^{*}=90^{\circ} .
\end{gathered}
$$




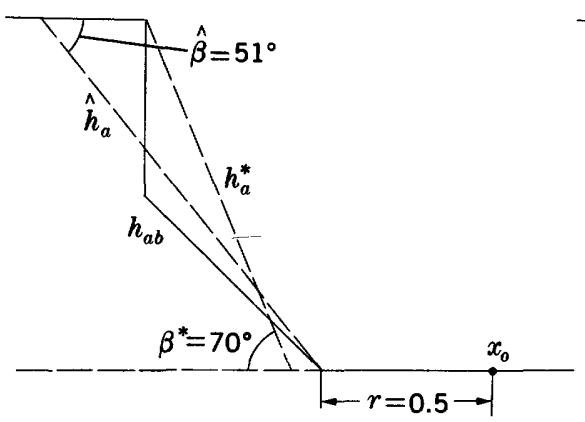

$A$

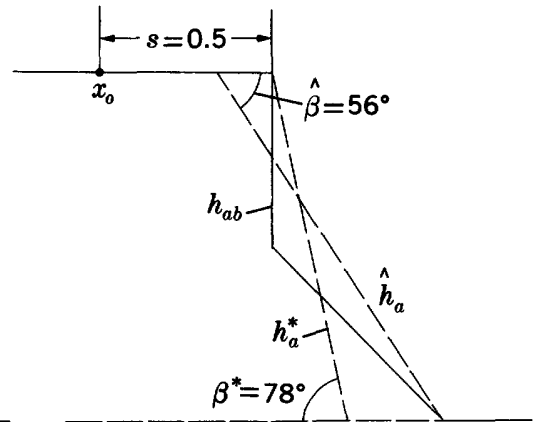

$B$

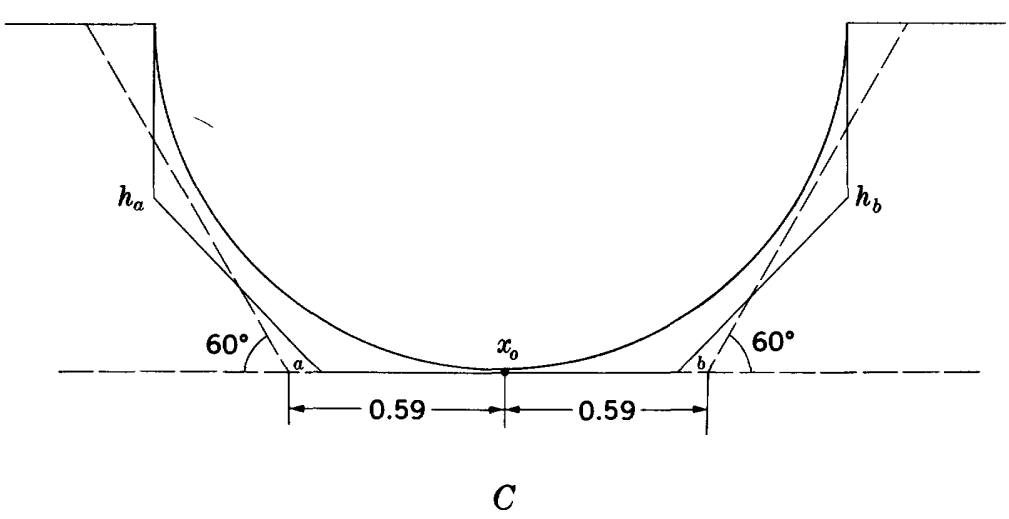

Figure 17.-Numerical examples of equivalent slopes.

By inequality 75 :

and hence:

$$
D^{*}\left(90^{\circ}, 0.38\right)<D(0.38)<\hat{D}\left(55^{\circ}, 0.38\right),
$$

$$
1.23<q\left(x_{0}\right)<1.30,
$$

where inequality 77 is obtained by adding $q^{\prime}=1.38$ to the values of $D^{*}$ and $\hat{D}$ read from figure 15 .

In step 1 we found that the Jeffreys approximation gave $q^{\prime}{ }_{a b}=1.38$ which would not have been very satisfactory. However, in this example simply adding the independent exact solutions for the two 
parts of $h_{a b}$ gives a much better result. It can be taken directly from table 1 as:

$$
\Delta q\left(90^{\circ}, r=2\right)+\Delta q\left(45^{\circ}, r=1\right)=0.115+0.178=0.293 .
$$

Example 2.-If the point $x_{0}$ were only 0.1 of the height of $h_{a b}$ from the toe, then:

Step 1 (pl. 2) : $\Delta q^{\prime}{ }_{a b}(r=0.1)=\Delta q^{\prime}\left(90^{\circ}, r=0.2\right)+\Delta q^{\prime}\left(45^{\circ}, r=0.2\right)$

$$
=0.27+0.57=0.84 \text {. }
$$

Step 2: Choosing $\hat{h}_{a}$ through the toe, $\hat{r}=0.1, \Delta q^{\prime}=0.84$ and plate 2 gives:

$$
\hat{\beta}=50^{\circ} \text {. }
$$

Choosing $h_{a}^{*}$ through the brink, $r^{*}+\cot \beta^{*}=0.6$ and figure 17 gives:

$$
\beta^{*}=69^{\circ}, r^{*}=0.60-\cot \beta^{*}=0.22 \text {. }
$$

Step 3: From plate 1:

that is:

$$
\begin{gathered}
\Delta \hat{q}\left(50^{\circ}, r=0.1\right)=0.68, \\
\Delta q^{*}\left(69^{\circ}, r=0.22\right)=0.52, \\
1.52<q_{a b}(r=0.1)<1.68 .
\end{gathered}
$$

The Jeffreys approximation yielded 1.84, and the sum of the exact solutions for the component slopes gives (pl. 1):

$$
q_{a b} \sim 1+\Delta q\left(90^{\circ}, r=1.2\right)+\Delta q\left(45^{\circ}, r=0.2\right)=1+0.18+0.46=1.64 .
$$

As in the previous example the sum of the independent exact solutions gives a satisfactory result.

Example 3.-If the point $x_{0}$ were one-half of a slope height behind the brink (fig. $17 B$ ), we would have (table 2 or pl. 2):

$\Delta q^{\prime}{ }_{a b}(s=0.5)=\Delta q^{\prime}(90, s=1)+\Delta q^{\prime}(45, s=1)=-0.538$.

Choosing $h_{a}^{*}$ through the brink and $\hat{h}_{a}$ through the toe from plate 2, we obtain:

$$
\beta^{*}=78^{\circ}, s^{*}=0.5, \hat{\beta}=55^{\circ} \hat{s}=1-\cot 55^{\circ}=0.30,
$$

and plate 1 yields:

$$
q^{*}\left(x_{0}\right)=0.44, \hat{q}\left(x_{0}\right)=0.47
$$




\section{Hence:}

$$
0.44<q_{a b}(s=0.5)<0.47 \text {. }
$$

From equation 79 we had for the Jeffreys approximation:

$$
q_{a b}^{\prime}=0.46 \text {. }
$$

The sum of the anomalies from slope components independently yields (table 2):

$$
q_{a b} \sim 1+\Delta q(90, s=1)+\Delta q(45, s=1)=0.34 .
$$

Thus, in this example, the Jeffreys approximation is satisfactory whereas the sum of component contributions is not, a result easily anticipated from figure 16.

Example 4.-The case $s=0.1$ yields:

$$
\Delta q^{\prime}{ }_{a b}(s=0.1)=\Delta q^{\prime}\left(90^{\circ}, s=0.2\right)+\Delta q^{\prime}\left(45^{\circ}, s=0.2\right)=-2.16 .
$$

From plate 2 we obtain:

$$
\begin{gathered}
\beta^{*}=84^{\circ}, s^{*}=0.1, \\
\hat{\beta}=60^{\circ}, \hat{s}=0.600-\cot 60^{\circ}=0.023
\end{gathered}
$$

and plate 1 and relation 71 yield:

$$
0.11<q_{a b}(s=0.1)<0.13 .
$$

In this example the Jeffreys approximation yields:

$$
q_{a b}^{\prime}=-1.16,
$$

and the sum of exact solutions for slope components (pl. 2):

$$
q_{a b} \sim 1+\Delta q\left(90^{\circ}, s=0.2\right)+\Delta q\left(45^{\circ}, s=0.2\right)=-0.36 .
$$

The second two results are, of course, physically impossible as $q_{a b}$ cannot be a negative quantity.

In the last example $x_{0}$ was very close to the brink where, as we have seen, the left side of inequality 71 might not be rigorously correct. If we had chosen $\beta^{*}=90^{\circ}$ instead of $84^{\circ}$, the same lower limit to two significant figures in inequality 83 would have resulted. If, however, we are concerned about the lower limit given by inequality 71 in cases like this, we can resort to simple geometric bracketing which yields:

$$
q\left(90^{\circ}, s=0.1\right)<q_{a b}(s=0.1),
$$


and from plate 1:

$$
0.08<q_{a b}(s=0.1) .
$$

This result demonstrates that the lower limit of 0.11 in inequality 83 cannot be much in error.

Example 5.-As a final example we consider the topographic anomaly at the center of a symmetrical valley whose walls have the same shape as the figure of the previous examples. The floor is assumed to be flat over a distance equal to the valley's depth (fig. 17C). We shall use the notation of inequality 44 , referring to one valley wall as $h_{a}$ and the other as $h_{b}$. From the first example (inequality 76):

$$
\Delta q_{a}\left(x_{0}\right)=\Delta q_{b}\left(x_{0}\right)=0.27 \pm 0.02 \text {. }
$$

From plates 1 and 2 it is seen that the plane slope equivalent to $h_{a}$ at $x_{0}$, which yields $\Delta q=0.27$, is $\beta=60^{\circ}, r=0.59$, that is:

$$
\begin{gathered}
\Delta q^{\prime}\left(60^{\circ}, r=0.59\right)=0.38, \\
\Delta q\left(60^{\circ}, r=0.59\right)=0.27=\Delta q_{a}\left(x_{0}\right) \pm 0.02=\Delta q_{b}\left(x_{0}\right) \pm 0.02 .
\end{gathered}
$$

The effect of the \pm 0.02 on the interaction is of higher order. Hence, from plate 1:

$$
\Delta q_{a}(b)=\Delta q_{b}(a)=\Delta q\left(60^{\circ}, r=1.18\right)=0.12 .
$$

Applying inequality 44:

$$
\begin{array}{r}
0.27 \pm 0.02+0.27 \pm 0.02<\Delta q\left(x_{0}\right)<(27 \pm 0.02+0.27+0.02) \\
{[1+0.12+0.01+* * *], 0.54+0.04<\Delta q\left(x_{0}\right)<0.61+0.04} \\
1.50<q\left(x_{0}\right)<1.65 .
\end{array}
$$

The circular valley of the same depth satisfies the conditions in relations 53 and 54a for an upper limit by geometric bracketing. The heat flow through its center is known to be 2.0 which is consistent with inequalities 84 and 54b.

\section{APPROXIMATING WITH A SINGLE EQUIVALENT PLANE SLOPE, $\bar{h}_{\mathrm{a}}$}

The bracketing procedure of the foregoing examples is generally more elaborate than is warranted by most geothermal applications, although it is necessary to investigate the limitations of the method. By inspection of the real slope, an intermediate angle, $\bar{\beta}$, for an approximating equivalent slope generally can be selected. It should be weighted in favor of the part of the slope closer to the station, although the result is rather insensitive to the choice, as can be determined with a little experimentation. Once $\bar{\beta}$ is selected, the result 
can be read directly from figures 15 or 16 or the coordinate $\bar{r}$ or $\bar{s}$ of $h_{a}$ can be determined from plate 2 and the result obtained from plate 1. In the foregoing examples, $\bar{\beta}=60^{\circ}$ would have led to results near the midpoint of the bracketing intervals, probably within a few percent of the exact values. (See numerical example, p. E63, eq 103.)

\section{STATIONS ON GENTLY SLOPING SURFACES}

Many of the results of the previous sections apply only to stations lying on geometrically horizontal surfaces, although these stations may be very close to steep and irregular slopes. The earth's surface cannot be considered geometrically horizontal over extended areas, but much of it (in the ocean basins at least) is inclined at angles of less than a degree or two. Although slope angles may change very rapidly near the toe and brink of topographic scarps, the distant transition to horizontalness is generally gradual. Hence, many heat-flow stations requiring topographic correction will lie on gently sloping surfaces adjacent to bold features. We must consider how to apply the foregoing results to stations on such surfaces.

For this purpose we consider the heat flow at $x=0$ on the gently sloping part of the surface $h_{a b}$ (fig. 18). We should like to establish conditions under which the heat-flow anomaly can be computed without appreciable error from the modified surface, $h_{a}$, which contains a horizontal part extending a distance $l / 2$ on both sides of the station.

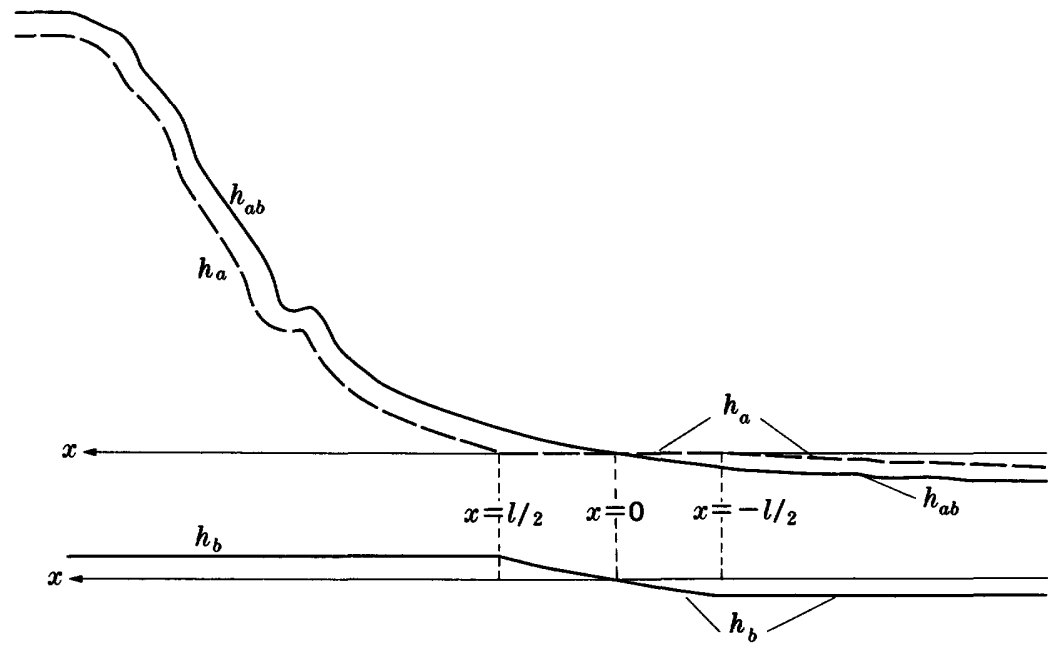

Fraure 18.-General slope $h_{a b}$ is flattened in the neighborhood of the station $(x=0)$ by subtracting the increment $h_{b}$ to form the modified slope $h_{a}$. 
We define $h_{a}$ by:

where:

$$
h_{a}=h_{a b}-h_{b}
$$

Hence:

$$
\begin{aligned}
h_{b}(x) & =h_{a b}(x),|x|<\frac{l}{2} \\
& =h_{a b}\left(\frac{l}{2}\right), x>\frac{l}{2} \\
& =h_{a b}\left(-\frac{l}{2}\right), x<-\frac{l}{2} .
\end{aligned}
$$

$$
\begin{aligned}
h_{a}(x) & =h_{a b}(x)-h_{a b}\left(-\frac{l}{2}\right), x<-\frac{l}{2} \\
& =0,|x|<\frac{l}{2} \\
& =h_{a b}(x)-h_{a b}\left(\frac{l}{2}\right), x>\frac{l}{2} .
\end{aligned}
$$

The difference between the heat-flow anomaly at $x=0$ on the surface $h_{a b}$ and that on the (locally) flattened surface $h_{a}$ can be represented by the exact expression:

$$
\begin{aligned}
& \Delta q_{a b}(0)-\Delta q_{a}(0)=\frac{1}{\pi} \int_{-\infty}^{+\infty} \frac{h_{a b}\left(1+e_{a b}\right)-h_{a}\left(1+e_{a}\right)}{x^{2}} d x \\
& =\int_{-\infty}^{+\infty} \frac{h_{b}\left(1+e_{a b}\right)+h_{a} \Delta e}{x^{2}} d x \\
& =\frac{1}{\pi}\left\{\int_{-\frac{l}{2}}^{+\frac{l}{2}} \frac{h_{b}\left(1+e_{a b}\right)}{x^{2}} d x+\int_{-\infty}^{-\frac{l}{2}} \frac{h_{b}\left(-\frac{l}{2}\right)\left[1+e_{a b}\left(-\frac{l}{2}\right)\right]}{x^{2}} d x\right. \\
& +\int_{-\infty}^{-\frac{l}{2}} \frac{\left.\left.+\int_{b}^{\infty}\left(-\frac{l}{2}\right)\left[e_{a b}(x)-e_{a b}\left(-\frac{l}{2}\right)\right]_{\frac{l}{2}} \frac{l}{2}\right)\left[1+e_{a b}\right)\right]_{d}}{x^{2}} d x+\int_{\frac{l}{2}}^{\infty} \frac{h_{b}\left(\frac{l}{2}\right)\left[e_{a b}(x)-e_{a b}\left(\frac{l}{2}\right)\right]_{d}}{x^{2}} d x \\
& \left.+\int_{-\infty}^{-\frac{l}{2}} \frac{h_{a} \Delta e}{x^{2}} d x+\int_{\frac{l}{2}}^{\infty} \frac{h_{a} \Delta e}{x^{2}} d x\right\} .
\end{aligned}
$$

The seven integrals in the last expression on the right-hand side of equation 87 require discussion. The first integral represents the direct effect of reference-plane temperatures in $|x|<\frac{l}{2}$ on heat flow at the 
station $(x=0)$. The second and fourth integrals taken together represent the effect of the contribution to reference-plane temperature in $x<-\frac{l}{2}$ of the sliver of height $\mathrm{h}_{b}\left(-\frac{l}{2}\right)$. The second integral contains the first-order effect, and what will generally be an overestimation of the second-order effect of the sliver. The expression in brackets in the fourth integral vanishes at $x=-\frac{l}{2}$ and hence the integral can be viewed as equivalent to the effects of subdued relief of height $h_{b}\left(-\frac{l}{2}\right)$ at a distance of $\frac{l}{2}$ from the station. The third and fifth integral are analogous to the second and fourth. In the sixth and seventh integrals $\Delta e$ is of the order of $\Delta q_{b}$ which, for small $h_{b}$, is appreciably different from zero only at $x=\frac{l}{2}+$ and $x=-\frac{l}{2}-$. At these points $h_{a}$ passes continuously to zero. Hence the last four integrals are of higher order, and they will generally be small relative to the first three integrals when $2\left|h_{b}\left( \pm \frac{l}{2}\right)\right| l^{-1}$ is small.

Inasmuch as $h_{b}$ is assumed to be gently sloping and smooth, it will generally be possible to represent it and $e_{a b}$ in $|x|<\frac{l}{2}$ by a few terms of a Maclaurin's series.

$$
\begin{aligned}
& h_{b}(x)=x h_{b}^{\prime}+\frac{1}{2} x^{2} h_{b}^{\prime \prime}+\frac{1}{6} x^{3} h_{b}^{\prime \prime \prime}+* * * \\
& e_{a b}(x)=e_{a b}(0)+x e_{a b}^{\prime}+\frac{1}{2} x^{2} e_{a b}^{\prime \prime}+* * *
\end{aligned}
$$

where the derivatives are evaluated at $x=0$. When these expressions are substituted into the first three integrals of equation 87 , only the even order derivatives of the reference-plane temperature contribute. We can replace $h_{b}$ with $h_{a b}$ as they are identical over the range of integration:

$$
\Delta q_{a b}(0)-\Delta q_{a}(0)=\frac{l}{\pi}\left[h_{a b}^{\prime \prime}\left(1+e_{a b}(0)\right)+2 h_{a b}^{\prime} e^{\prime}\right]+0\left(l^{3} \frac{d^{4}}{d x^{4}}\left(h_{a b}\left[1+e_{a b}\right]\right)\right) .
$$

Inasmuch as $h_{b}$ is small, the mean gradient in the relief, $\left(1+e_{a b}\right)$, can be replaced by $q_{a b}$ the heat flow (and gradient) at the surface. Hence:

$$
q_{a b}(0)-q_{a}(0) \cong \frac{l}{\pi}\left[q_{a b}(0) \frac{d^{2} h_{a b}}{d x^{2}}+2 \frac{d h_{a b}}{d x} \frac{d q_{a b}}{d x}\right]
$$


Equation 90 represents the "flattening error." It is the heat-flow error that can be expected where a gently sloping smooth part of a general two-dimensional surface, $h_{a b}$, is replaced by a horizontal plane segment of width $l$ centered at the station. The relief beyond $x= \pm l / 2$ is adjusted upward or downward to eliminate discontinuities at $x=$ $\pm l / 2$. The error is independent of the local heat flow if the curvature is negligible, and independent of the lateral gradient of heat flow if the slope at the station is negligible. The curvature term takes the sign of the curvature. The slope term is positive if the heat flow is decreasing downslope and negative otherwise.

For numerical applications it is more convenient to have equation 90 in finite form. Let $\beta(x)$ denote the slope of the tangent to $h_{a b}(x)$ measured clockwise from negative $x$, and let:

$$
\begin{gathered}
\Delta \beta=\beta\left(\frac{l}{2}\right)-\beta\left(-\frac{l}{2}\right), \\
\delta q=\frac{q_{a b}\left(\frac{l}{2}\right)-q_{a b}\left(-\frac{l}{2}\right)}{q_{a b}(0)} .
\end{gathered}
$$

Then equation 90 can be written:

$$
\frac{q_{a b}(0)-q_{a}(0)}{q_{a b}(0)}=\frac{1}{\pi}[\tan \Delta \beta+2 \delta q \tan \beta(0)] .
$$

If the change in slope $(\Delta \beta)$ over the flattened interval is less than $2^{\circ}$, the curvature term will not contribute more than 1 percent to the error; if it is less than $5^{\circ}$, the contribution will be less than about $2 \frac{1}{2}$ percent. In general $\delta q$, the relative change in heat flow across the flattened interval, will not be known, but inspection of the slope will normally permit an estimate of its order of magnitude. If $\delta q$ is 50 percent, a rather extreme case, the second term will contribute about 1 percent to the error if the slope at the station is $2^{\circ}$. If $\delta q$ is 20 percent, a $5^{\circ}$ slope will contribute 1 percent.

Equation 91 probably should not be used for $\Delta \beta$ and $\beta_{0}$ much larger than $5^{\circ}$ or $6^{\circ}$ because of the condition, $2\left|h_{b}\left( \pm \frac{l}{2}\right)\right| l^{-1}<<1$, required to neglect the last four integrals in equation 87. When $\delta q$ is large, $q_{a b}(x)$ is likely to have considerable curvature in $|x|<\frac{l}{2}$. It is seen from equation 89 that the lowest order term containing curvature of $e_{a b}(x)$ occurs as a product with $h^{\prime \prime}$. Thus, if $\Delta \beta$ is very small, substantial curvature in $q_{a b}$ can probably be tolerated in equation 91 . If not, it is probably best to restrict its application to cases in which $q \delta$ does not exceed about 25 percent. This will include most cases of interest. 


\section{NUMERICAI EXAMPLE}

To illustrate the use of the flattening procedure with the approximating schemes of the preceding sections, we consider the monocline of Jaeger and Sass (1963, eq 11 with $\alpha=1.01$ ). The heat flow will be estimated at two points: (1) In the vicinity of the toe at $u=-1.5$ (fig. 19), and (2) in the vicinity of the brink at $u=+0.55$ (fig. 20).

Point 1: Station at $u=-1.5$ (fig. 19). The slope is flattened from $u=-1.3$ to $u=-1.7$ in which interval $\Delta \beta=1.6^{\circ}, \beta_{0}=4.5^{\circ}$, and $\delta q$ is obviously small. (Its actual value is 0.02 .)

The Jeffreys approximation of the effect of relief to the left and right of the flattened interval is obtained by numerical integration, for example, by adding effects of many small plane slopes.

$$
\begin{gathered}
\Delta q_{a}^{\prime}=0.21 \\
\Delta q_{b}^{\prime}=-0.035
\end{gathered}
$$

Equivalent slopes for the relief to the left of the station are (pls. 1 and 2):

$$
\begin{aligned}
& \hat{h}\left(\beta=12.5^{\circ}, r=0.24\right), \\
& h^{*}\left(\beta=38^{\circ}, r=0.90\right) .
\end{aligned}
$$
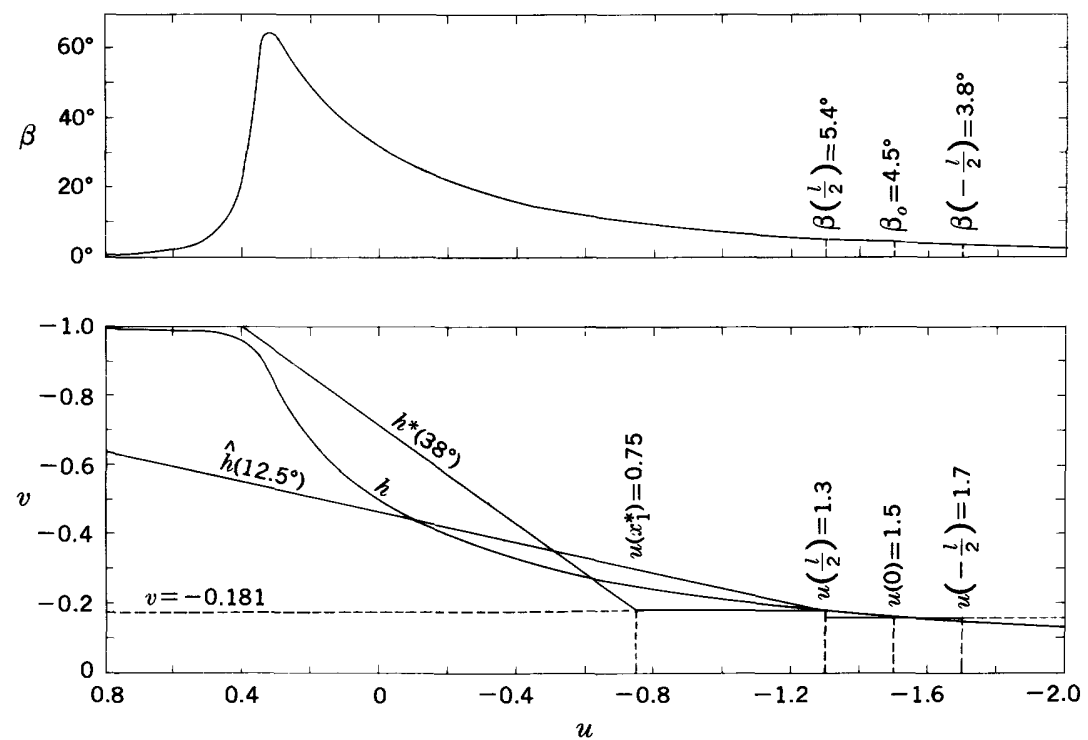

Figure 19.-Numerical example 1, stations on gently sloping surfaces. Monocline of Jaeger and Sass with $\alpha=1.01$ (lower scale) and its slope angle (upper scale). 
Hence:

$$
\begin{gathered}
\Delta q^{*}\left(38^{\circ}, r=0.9\right)<\Delta q_{a}<\Delta \hat{q}\left(12.5^{\circ}, r=0.24\right) \\
0.18<\Delta q_{a}<0.21 .
\end{gathered}
$$

It is known that (fig. 15):

$$
\Delta q_{b}^{\prime}=\Delta q_{b}=-0.035 .
$$

For the estimation of interaction, $\Delta q_{b}$ can be represented by:

$$
\Delta q\left(\beta=2^{\circ}, s=1.33\right) \text {. }
$$

Applying inequality $49 a, b$ :

$$
\begin{aligned}
0.21-0.035>\Delta q & >0.18[1-0.02]-0.035[1+0.15] \\
\Delta q & =0.155 \pm 0.02 .
\end{aligned}
$$

The exact result is 0.161 , and the Jeffreys approximation is 0.175 . The good agreement between the two could have been anticipated from figure 15; the relief to the left is concave at the toe and the interaction is small.

Point 2: Station at $u=+0.55$ (fig. 20). The slope is flattened from $u=0.50$ to $u=0.60$ (that is, $l=0.10$ ) (fig. 20). In this interval $\Delta \beta=-3.1^{\circ}, \beta_{0}=3.4^{\circ}$ and $\delta q$ is large, as this is the region in which $q(x)$ has its largest gradients. (Its actual value is about 25 percent.) The contribution of the slope term to the flattening error (eq 91) is $+0.01 q\left(x_{0}\right)$ and the curvature term contributes $-0.015 q\left(x_{0}\right)$. As we shall see, $q\left(x_{0}\right) \sim 0.5$, and hence the flattening error amounts to only a few tenths of 1 percent of the regional heat flow. The results for this example are:

exact solution: $\quad \Delta q\left(x_{0}\right)=-0.58$.

Jeffreys approximation: $\quad \Delta q^{\prime}\left(x_{0}\right)=-0.675$.

$$
\begin{aligned}
& \Delta q_{b}^{\prime}=-0.69 . \\
& \Delta q_{a}^{\prime}=+0.015 . \\
& \beta\left(\frac{l}{2}\right)=2.3^{\circ} . \\
& \beta\left(-\frac{l}{2}\right)=5.4^{\circ} .
\end{aligned}
$$

We first assume that the information in equations $96 \mathrm{a}-\mathrm{d}$ is not available and see what can be done by simple geometric bracketing. 

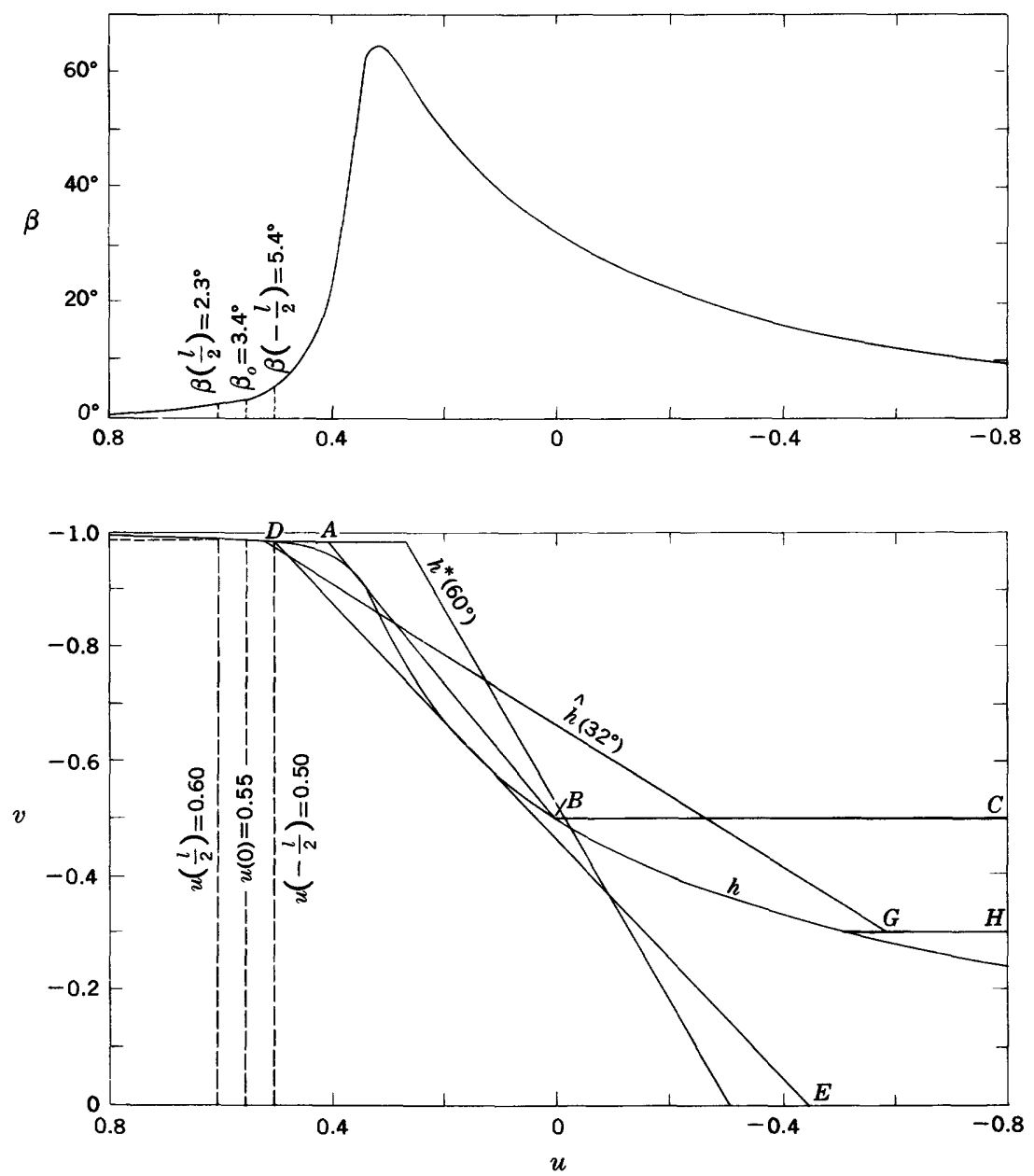

Figure 20.-Numerical example 2, stations on gently sloping surfaces.

The effect of relief to the right of the station is overestimated by that of the plane slope $A B C$ and underestimated by the slope $D E$ (fig. 20). Thus:

$$
\Delta q\left(\beta=50^{\circ}, s=0.28\right)>\Delta q_{b}>\Delta q\left(45^{\circ}, s=0.051\right),
$$

and plate 1 yields:

$$
-0.50>\Delta q_{b}>-0.69 \text {. }
$$

The relief to the left is positive, and it is overestimated by the plane slope tangent to the relief at $l / 2$,

$$
\Delta q\left(\beta=2.3^{\circ}, r=3.6\right)>\Delta q_{a}>0,
$$


and from plate 1:

$$
+0.02>\Delta q_{a}>0 .
$$

From inequality $49 a, b$, the interaction will not exceed -0.01 . Hence:

$$
-0.48>\Delta q\left(x_{0}\right)>-0.70 \text {. }
$$

In practice, one would be more likely to approximate the relief with plane slopes rather than take the extreme bracketing configurations. This method would generally lead to an estimate near the midpoint of inequality 99 and within a few percent of the exact value (eq 96a), considerably better than the Jeffreys approximation without recourse to numerical integration.

If the Jeffreys approximation, equation $96 \mathrm{~b}$, c, and $d$, is computed, the method of equivalent slopes can be applied. The present example illustrates a complication because, although the curvature is negative near the station, the relief to its right is not convex at the brink because of the long gently sloping toe. It is not possible to obtain the upper limit, $\Delta \hat{q}$, by passing $\hat{h}$ through the brink $x=-\frac{l}{2}$. We therefore replace the part of the slope below $-V=0.3$ with the horizontal surface $G H$ (fig. 20). By the theorem on geometric bracketing the modified surface will cause an algebraically larger anomaly than the true surface, and $\hat{h}$ for the modified surface will yield the upper limit required. $\Delta q_{b}^{\prime}$ for the modified surface is -0.65 . We select $\beta^{*}=60^{\circ}$ and from plates 1 and 2 obtain:

$$
\begin{aligned}
\Delta \hat{q}\left(32^{\circ}, s\right. & =0.06)>\Delta q_{b}>\Delta q^{*}\left(60^{\circ}, s=0.23\right) \\
& -0.54>\Delta q_{b}>-0.61 .
\end{aligned}
$$

From figure 16 it is known that:

$$
\Delta q_{a}=\Delta q_{a}^{\prime}=0.015 \text {. }
$$

Adding equation 101 to inequality 100 and subtracting 0.01 (an upper limit to the interaction) from the right-hand side yields:

$$
-0.525>\Delta q\left(x_{0}\right)>-0.605 \text {. }
$$

This is to be compared with the exact result, -0.58 (eq 96a), and the Jeffreys approximation, -0.675 (eq $96 \mathrm{~b}$ ).

The bracketing procedure has been somewhat belabored for purposes of illustration, but in practice the calculation is much simpler. The upper part of the right-hand relief $\left(h_{b}\right)$ has an average slope of about $45^{\circ}$. Approximating $h_{b}$ by the equivalent $45^{\circ}$ slope, $\bar{h}_{b}$, we obtain directly from figure 16 :

$$
\overline{\Delta q_{b}} \cong \Delta q_{b}{ }^{\prime}+D\left(45^{\circ},-0.69\right)=-0.69+0.11=-0.58 \text {. }
$$


Adding $\Delta q_{b}$ (eq. 101) and subtracting 0.005 for interaction yields:

$$
\Delta q\left(x_{0}\right) \cong-0.57,
$$

which agrees well with the exact result (eq. 96a). It is seen from figure 16 that if $60^{\circ}$ had been chosen instead of $45^{\circ}$, for the approximating equivalent slope, the corresponding result would have been 3 percent lower; a $30^{\circ}$ equivalent slope would have made it only 3 percent higher.

\section{VARIATION OF THE TOPOGRAPHIC ANOMALY WITH DEPTH}

To this point the discussion has been concerned only with the flux of heat across the surface and therefore has focused only on the limiting value of the thermal gradient at zero depth $(z=0)$. Even in oceanic measurements of geothermal flux, however, temperature gradients are determined from observations to finite depths (1-10 m). It is necessary to determine the conditions under which topographic anomalies computed for the surface can be applied to gradients determined beneath it without appreciable error, that is, conditions under which the gradient anomaly may be treated as superficial.

It is probably worth noting at the outset that inasmuch as the temperature satisfies Laplace's equation, and the solid surface is isothermal, the second derivative of temperature must vanish in every direction at the surface, wherever the surface has a continuous tangent. Hence:

$$
0=\frac{\partial^{2} \theta}{\partial z^{2}}=\frac{G}{K} \frac{\partial q}{\partial z}, z=0,
$$

and, in general, the heat flow is not changing with depth immediately below the surface. At reentrant corners where the surface heat flow is infinite, the vertical gradient of heat flow is negatively infinite so that finite heat flow occurs at finite depths.

To investigate depth variations of heat flow analytically we rewrite equation 17 in the form of equation 24 .

$$
\Delta q\left(x_{0}, z\right)=\frac{1}{\pi} \int_{-\infty}^{+\infty} \frac{h(x)[1+e(x)]}{\left(x-x_{0}\right)^{2}} \Phi(x) d x,
$$

where:

$$
\begin{aligned}
\chi & =\left|\frac{x-x_{0}}{z}\right| \\
\Phi(\chi) & =\frac{1-\chi^{-2}}{\left(1+\chi^{-2}\right)^{2}}
\end{aligned}
$$




$$
\begin{aligned}
\Phi(\chi) & =1-\frac{3}{\chi^{2}}+\frac{5}{\chi^{4}}-{ }_{* * *}, \chi>1 \\
& =-\chi^{2}+3 \chi^{4}-{ }_{* * *}, \chi<<1 .
\end{aligned}
$$

Equation 106 is an exact expression for the effect of any twodimensional topographic surface, $h(x)$, on the vertical gradient at the point $\left(x_{0}, z\right)$. Although $e(x)$ is unknown, its physical interpretation is clear; it is the mean anomalous gradient in the relief at $x$.

Inspection of the form of the function $\Phi$ (fig. 21) and equation 106 points up a fundamental problem of attempting regionally meaningful measurements of thermal gradient at or near the surface. The function

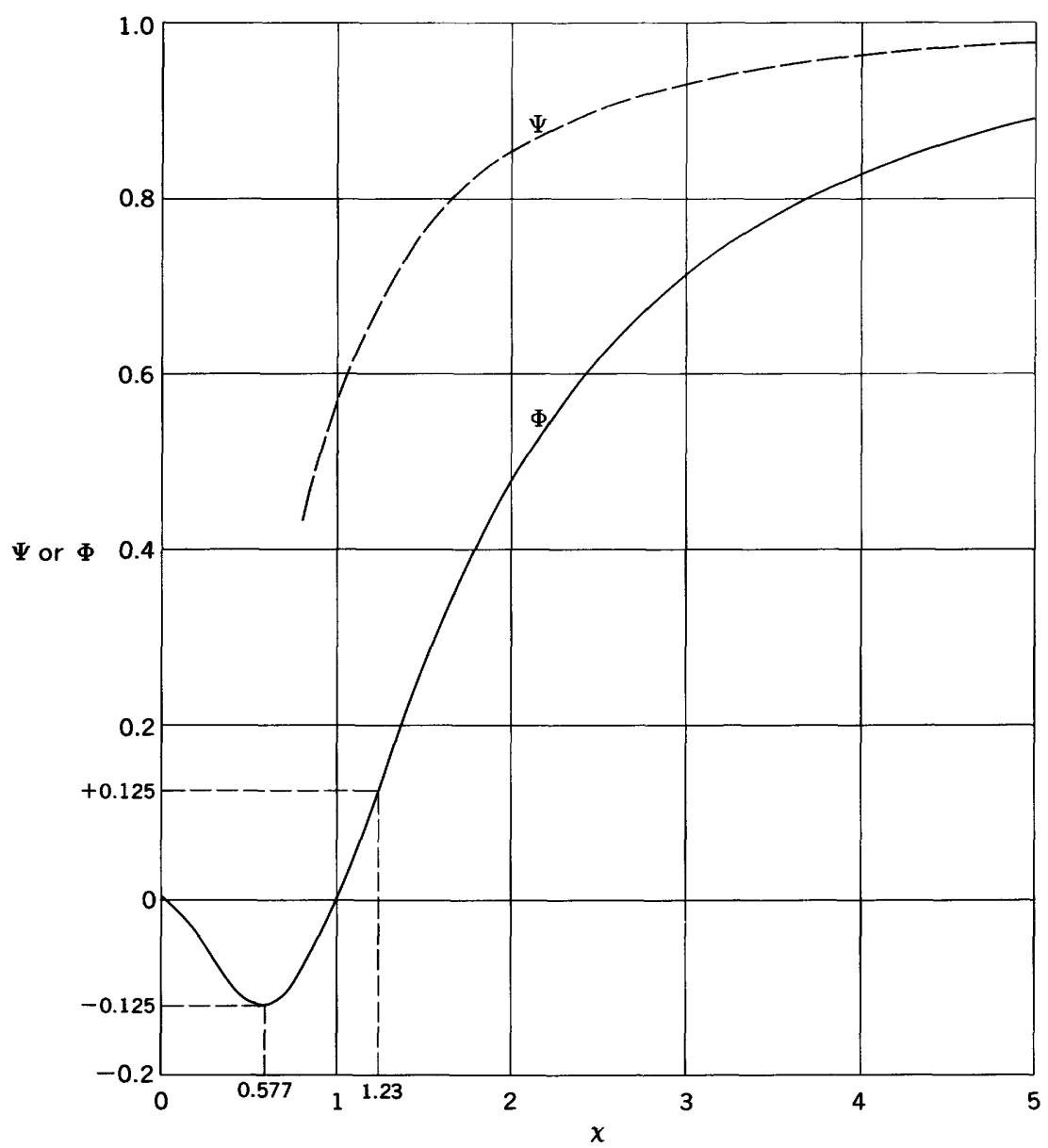

Figure 21.-The factors $\Phi$ and $\Psi$ for depth dependence of the integrand in expressions for the gradient anomaly. 
$\Phi$ greatly diminishes effects of topographic features whose horizontal distance from the station, $\left(x-x_{0}\right)$, is not large relative to the depth of observation, that is, relief for which $\chi$ is not large. It is these features specifically that can have a very great effect on the gradient at the surface because of the inverse square growth of the fraction in the integrand of equation 106. As $z$ approaches the surface $\Phi$ approaches unity for all $x$, and very small features very close to the station can have very large effects on the gradients. We shall investigate these effects.

For the general topography illustrated in figure 22, equation 106 can be rewritten:

$$
\begin{aligned}
\Delta q\left(x_{0}, z\right)=\frac{1}{\pi} \int_{x_{0}}^{x_{1}} \frac{h(x)[1+e]}{\left(x-x_{0}\right)^{2}} \Phi d x+\frac{1}{\pi} \int_{x_{1}}^{x_{2}} \frac{h(x)[1+e]}{\left(x-x_{0}\right)^{2}} \Phi d x+* * * \\
+\frac{1}{\pi} \int_{x_{1}^{\prime}}^{x_{0}} \frac{h(x)[1+e]}{\left(x-x_{0}\right)^{2}} \Phi d x+\frac{1}{\pi} \int_{x_{2}^{\prime}}^{x} \frac{h(x)[1+e]}{\left(x-x_{0}\right)^{2}} \Phi d x+\ldots * *
\end{aligned}
$$

The points $x_{i}$ (fig. 22) are selected in such a way that $h(x)$ does not change sign in any subinterval.

Inasmuch as the topographic anomaly cannot reverse the sign of the gradient, $(1+e)$ is positive over any finite interval. Therefore, the fractions in the integrands of equation 108 do not change sign, and the mean value theorem can be applied to each integral.

$$
\begin{aligned}
\Delta q(x, z)= & \frac{1}{\pi} \bar{\Phi}_{1} \int_{x_{0}}^{x_{1}} \frac{h(x)[1+e]}{\left(x-x_{0}\right)^{2}} d x+\frac{1}{\pi} \bar{\Phi}_{2} \int_{x_{1}}^{x_{2}} \frac{h(x)[1+e]}{\left(x-x_{0}\right)^{2}} d x+* * * \\
& +\frac{1}{\pi} \bar{\Phi}_{1}^{\prime} \int_{x_{1}^{\prime}}^{x_{0}} \frac{h(x)[1+e]}{\left(x-x_{0}\right)^{2}} d x+\frac{1}{\pi} \bar{\Phi}_{2}^{\prime} \int_{x_{2}^{\prime}}^{x_{1}^{\prime}} \frac{h(x)[1+e]}{\left(x-x_{0}\right)^{2}} d x+* * *,
\end{aligned}
$$

where:

and:

$$
\begin{aligned}
& \bar{\Phi}_{i}=\Phi\left(\bar{\chi}_{i}\right), \chi_{i}>\bar{\chi}_{i}>\chi_{i-1}, \\
& \bar{\Phi}_{i}^{\prime}=\Phi\left(\bar{\chi}_{i}^{\prime}\right), \chi_{i-1}^{\prime}>\bar{\chi}>\chi_{i}^{\prime},
\end{aligned}
$$

$$
\chi_{i}=\frac{\left|x_{i}-x_{0}\right|}{z} \text {. }
$$

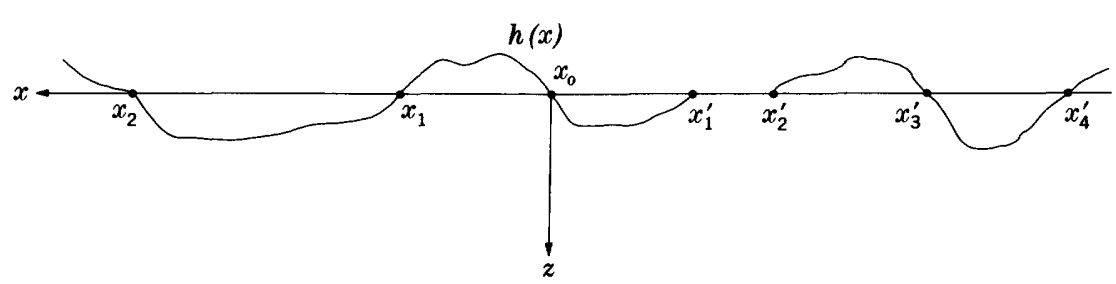

FIGURE 22.-General two-dimensional relief, $h(x)$, and the notation for equation 108. 
The barred $\chi^{\prime}$ s are the ones that give the appropriate mean value of $\Phi$ in each integral.

The form of $e(x)$ in each interval of equation 109 depends upon the form of $h(x)$ in every other interval because of interaction between the topographic elements. Hence a single term of equation 109 cannot be identified with the complete effect of topography in its interval unless the interval contains all the nonvanishing topography. Some special cases of equation 109 will be discussed.

CASE 1, AIL TOPOGRAPHY OF ONE SIGN: GENERAL

$$
(h(x) \geq 0 \text { or } h(x) \leq 0, \infty>x>-\infty)
$$

In this case equation 109 can be written:

$$
\Delta q\left(x_{0}, z\right)=\frac{1}{\pi} \bar{\Phi} \int_{-\infty}^{+\infty} \frac{h(x)[1+e]}{\left(x-x_{0}\right)^{2}} d x .
$$

The integral is an exact expression for the topographic anomaly at the surface. By equation 107a, $|\bar{\Phi}|<1$, and hence:

$$
\left|\Delta q\left(x_{0}, 0\right)\right|>\left|\Delta q\left(x_{0}, z\right)\right|, z>0 .
$$

Inequality 111 can be stated as a theorem: If the topographic relief is of one sign at the station $\left(\mathrm{x}_{0}\right)$, then the heat-flow anomaly caused by this relief at $\left(\mathrm{x}_{0}, \mathrm{z}\right)$ attains its greatest magnitude at the surface $\mathrm{z}=0$.

The theorem applies to the Jeffreys approximation as well as to the exact result. However, it does not apply in general to the discrepancy $\left(D\left(x_{0}, z\right)\right)$ between the two, as $e(x)$ can change sign where $h(x)$ does not. Hence, it is quite possible for the error in the Jeffreys approximation to be greater at depth than at the surface. It can be shown that the theorem applies also to the transient case if the change of $h(x)$ with time is of the same sign as $h$ (or is zero).

All the topography nonnegative $(h(x) \geq 0)$ could correspond to a station at the edge of a plane or beyond the toe of a scarp or a range of ridges. It could also represent a station in a thinly sedimented depression on a rocky ocean bottom. All the topography nonpositive may represent a station near the edge of a plateau or shelf, or perhaps one on the crest of ripple marks.

\section{CASE 2, RELIEF VANISHES NEAR THE STATION AND IS OF ONE SIGN ELSEWHERE$$
\left(h(x)=0, x_{1}>x>x_{1}^{\prime} ; h(x) \geq 0 \text { or } h(x) \leq 0, x>x_{1}, x<x_{1}^{\prime}\right)
$$

In this case:

$$
\Delta q\left(x_{0}, z\right)=\frac{1}{\pi} \bar{\Phi}_{1} \int_{x_{1}}^{\infty} \frac{h[1+e]}{\left(x-x_{0}\right)^{2}} d x+\frac{1}{\pi} \bar{\Phi}_{1}^{\prime} \int_{\infty}^{x_{1}^{\prime}} \frac{h[1+e]}{\left(x-x_{0}\right)^{2}} d x .
$$


Exclusive of the $\Phi$ 's, the right side of equation 112 represents the exact effect of the topography on heat flow at $\left(x_{0}, 0\right)$ even though the complete effects of each of the two elements of the topography cannot be identified individually with each of the terms. Without loss of generality we assume that the relief on positive $x$ is not farther from $x_{0}$ than that on negative $x$, that is:

$$
\chi_{1} \leqslant \chi_{1}^{\prime}
$$

If the depth, $z$, of the observation point is less than the distance $\left|x_{1}-x_{0}\right|$ to the nearest relief:

$$
1>\bar{\Phi}_{1}, \bar{\Phi}_{1}^{\prime}>\Phi\left(\chi_{1}\right)>0, x_{1}>1,
$$

and from equation 112 :

or:

$$
\left|\Delta q\left(x_{0}, 0\right)\right|>\left|\Delta q\left(x_{0}, z\right)\right|>\Phi\left(x_{1}\right)\left|\Delta q\left(x_{0}, 0\right)\right|,
$$

$$
\frac{\Delta q\left(x_{0}, z\right)}{\Delta q\left(x_{0}, 0\right)}>\Phi\left(x_{1}\right)
$$

In oceanic applications the gradient is often determined from the difference in two measured temperatures; one taken near the sea bottom, and the other at some finite depth $(z)$ beneath it. Similarly, on the continents, the mean gradient is sometimes estimated from the differences between the local mean air temperature and the temperature at the bottom of a borehole. The departure of the mean gradient (or mean heat flow), determined in this way, from the regional value will be referred to as $\overline{\Delta q}\left(x_{0}, z\right)$.

$$
\begin{aligned}
\overline{\Delta q}\left(x_{0}, z\right) & =\frac{1}{z} \int_{0}^{z} \Delta q\left(x_{0}, z^{\prime}\right) d z^{\prime} \\
= & \frac{1}{\pi z} \int_{0}^{z}\left\{\int_{x_{1}}^{\infty} \frac{h[1+e]}{\left(x-x_{0}\right)^{2}} \Phi\left(\frac{x-x_{0}}{z^{\prime}}\right) d x+\int_{\infty}^{x^{\prime}} \frac{h[1+e]}{\left(x-x_{0}\right)^{2}} \Phi\left(\frac{\left|x-x_{0}\right|}{z^{\prime}}\right) d x\right\} d z .
\end{aligned}
$$

Imposing conditions 113 and 114 we can write:

$$
\overline{\Delta q}\left(x_{0}, z\right)>\left\{\frac{1}{z} \int_{0}^{z} \Phi\left(\frac{\left|x_{1}-x_{0}\right|}{z^{\prime}}\right) d z^{\prime}\right\} \frac{1}{\pi}\left\{\int_{x_{1}}^{\infty}+\int_{\infty}^{x_{1}^{\prime}}\right\} \frac{h[1+e]}{\left(x-x_{0}\right)^{2}} d x
$$

Hence:

$$
\frac{\overline{\Delta q}\left(x_{0}, z\right)}{\Delta q(x, 0)}>\Psi\left(x_{1}\right)
$$

where $\Psi(\chi)=\chi \int_{0}^{\frac{1}{\chi}} \frac{1-s^{2}}{1+s^{2}} d s=\chi\left[2 \tan ^{-1} \frac{1}{\chi}-\frac{1}{\chi}\right]$

$$
=1-\frac{2}{3} \frac{1}{\chi^{2}}+\frac{2}{5} \frac{1}{\chi^{4}}-\frac{2}{7} \frac{1}{\chi^{6}}+{ }_{* * *} \chi>1 \text {. }
$$


The function $\Psi$ is illustrated by the dashed curve in figure 21 .

Thus, for any topographic configuration $h(x)$, of one sign, whose distance from the station $\left|x_{1}-x_{0}\right|$ exceeds the depth of measurement (z), we have:

1. The ratio of the gradient anomaly at depth $z$ to that at the surface is greater than $\Phi\left(\frac{\left|x_{1}-x_{0}\right|}{z}\right)$ (inequality 115).

2. The ratio of the anomaly in the mean gradient (between the surface and depth $z$ ) to the gradient anomaly at the surface is greater than $\Psi\left(\frac{\left|x_{1}-x_{0}\right|}{z}\right)$ (inequality 116 and eq $117 \mathrm{a}, \mathrm{b}$ ).

If $z$ is 10 percent of the distance from the toe (or brink) of the nearest relief $\left(\chi_{1}=10\right)$, the topographic anomaly at depth $z$ differs by less than 3 percent from the value at the surface, and the mean value of the anomaly from the surface to depth $z$ is within 0.7 percent of the surface value. If $z$ is 20 percent of the distance to the nearest relief $\left(\chi_{1}=5\right)$, the gradient anomaly varies between the surface and depth $z$ by less than 11 percent, but its mean differs from the surface value by less than 2.6 percent. The function $\Phi$ falls rapidly for $\chi<5$ and hence there is no assurance that the surface correction is a good approximation to the correction applicable at depth $z$ for relief at a distance less than four or five times the depth. However, when $\chi_{1}$ is only 2 , the function $\Psi$ is still 0.86 , and hence the mean gradient anomaly in $(0, z)$ can be represented reasonably well by the surface correction for relief that extends as close to the station as twice the depth.

CASE a, RELIEF NEAR THE STATION IS OF ONE SIGN AND IT VA.NISHES ELSEWHERE $\left(h(x) \geq 0\right.$ or $\left.h(x) \leq 0, x_{1}<x<x_{1}^{\prime} ; h(x)=0, x>x_{1}, x<x_{1}^{\prime}\right)$

In this case:

$$
\Delta q\left(x_{0}, z\right)=\frac{1}{\pi} \bar{\Phi} \int_{x_{1}}^{x_{1}} \frac{h[1+e]}{\left(x-x_{0}\right)^{2}} d x .
$$

Denoting by $\left|\Phi_{m}\right|$ the greatest value of $|\Phi|$ in $\left(x_{1}, x_{1}^{\prime}\right)$ we obtain:

$$
\frac{\Delta q\left(x_{0}, z\right)}{\Delta q\left(x_{0}, 0\right)}<\left|\Phi_{m}\right| .
$$

Without loss of generality, we assume that the most distant relief occurs at $x_{1}$. If $\left(x_{1}-x_{0}\right)$ is more than $1.23 z$ then it is seen from figure 21 that:

$$
\left|\Phi_{m}\right|=\Phi\left(\chi_{1}\right)>0.125, \chi_{1}>1.23 \text {. }
$$


The effect of relief extending outward to five times the depth $\left(\chi_{1}=5\right)$ cannot exceed 88 percent of the surface value (fig. 21). At a depth equal to half the maximum distance to the relief $\left(\chi_{1}=2\right)$, the topographic correction cannot exceed 48 percent of the surface value.

Those parts of the relief whose horizontal distance from the station is less than the depth cause gradient anomalies of sign opposite that of the surface value. Integration over the changing sign of $\Phi$ will generally result in much more rapid depth decay of the close-in topographic anomaly than is indicated in the limits imposed by inequality 120. Consider, for example, the case in which the effective relief $(h[1+e])$, equation 118 , decreases in proportion to the square of its distance as the station is approached. If $x_{1}=-x_{1}^{\prime}=2 z$, integration of equation 106 yields. :

$$
\frac{\Delta q\left(x_{0}, z\right)}{\Delta q\left(x_{0}, 0\right)}=0.09 .
$$

This 9 percent is to be compared with the 48 percent given by inequality 120. If more relief occurs closer to the station, the reduction of the anomaly with depth is even greater. If $h(1+e)$ is uniform from $|x|=2 z$ to $|x|=0.1 z$, the anomaly at depth is only 3 percent of the value applicable at the surface (and of opposite sign). Thus, the effect of relief extending outward a distance $2 z$ from the station can generally be expected to be an order of magnitude less at depth $z$ than at the surface.

If $\chi_{1}<0.577$, then it is again true that $\left|\Phi_{m}\right|=\left|\Phi\left(\chi_{1}\right)\right|$ and inequality 119 can be written (equation 107):

$$
\left|\frac{\Delta q\left(x_{0}, z\right)}{\Delta q\left(x_{0}, 0\right)}\right|<\chi_{1}^{2}-3 \chi_{1}^{4}+_{* * *}, \chi_{1}<0.577 \text {. }
$$

Thus, at depths an order of magnitude greater than the width of a close-in feature of one sign, the topographic anomaly is reduced at least two orders of magnitude (and changed in sign).

\section{CASE 4, TOPOGRAPHY OF BOTH SIGNS: GENERAL}

If $h(x)$ is composed of $m$ positive parts in the intervals $\left(x_{i}, x_{i+1}\right), i=1$, $2,3, *^{*} * m$, and $n$ negative parts in the intervals $\left(x_{j}, x_{j+1}\right), j=1,2$, $3 * * * n$, then equation 109 can be written:

$$
\Delta q\left(x_{0}, z\right)=\frac{1}{\pi} \sum_{i=1}^{m} \bar{\Phi}_{i} \int_{x_{i}}^{x_{i}+1} \frac{h(x)[1+e]}{\left(x-x_{0}\right)^{2}} d x+\frac{1}{\pi} \sum_{j=1}^{n} \bar{\Phi}_{j} \int_{x_{j}}^{x_{j}+1} \frac{h(x)[1+e]}{\left(x-x_{0}\right)^{2}} d x,
$$

where the integration is performed in the direction of positive $x$. As before, if the $\Phi^{\prime}$ 's were set equal to unity, the series would give the 
exact value of $\Delta q\left(x_{0}, 0\right)$. However, unlike the former cases, $\Delta q\left(x_{0}, z\right)$ cannot now be expressed simply in terms of $\Delta q\left(x_{0}, 0\right)$ and bracketing values of $\Phi$, because the theorem stated from 111 no longer applies. For example, if the positive terms in equation 122 (the " $i$-terms") represent a very large feature far from the station, and the negative terms, a very small feature close to the station, they might cancel at the surface to give $\Delta q\left(x_{0}, 0\right)=0$. As $z$ increased, the influence of the negative terms $\left(\Phi_{j}\right.$, eq 122) would diminish very rapidly and $\Delta q\left(x_{0}, z\right)$ would become strongly positive.

If the interaction between the positive and negative elements of the topography is neglected, the anomaly at any depth can be estimated by considering the effects of each separately.

\section{CASE 5, VARIATION OF THE FLATTENING ERROR WITH DEPTH}

In the previous section it was shown that if the topography was gently sloping and smooth in the vicinity of a heat-flow station, in the sense that the surface and the heat flow through it could be represented by a few terms of Maclaurin's series, then the topographic anomaly at the surface could be computed by flattening the slope in the vicinity of the station. By introducing the factor $\Phi$ into the integrands of equation 87 it can be shown that the individual terms of the flattening error (eq 91) would be smaller in magnitude at $z>0$ than at the surface. If the interval is flattened for a distance $l / 2$ that exceeds five times the depth of the observation, the variation with depth of the last four integrals of equation 91 will generally be small.

\section{DISCUSSION}

It is now possible to return to the problem posed at the beginning of this section and define "superficial gradient" in terms of the topographic relief. We can refer to a gradient measurement at (or to) any depth $\lambda$ as superficial if the applicable topographic anomaly is approximated well by the value applicable at the surface. If we now denote by $x_{n}$ the horizontal distance from the station to the nearest point of the relief (flattened by the method of p. E56-E59 if necessary), then if the relief is of one sign, (a) gradient measurements at depth $\lambda$ are superficial if $\frac{\lambda}{x_{n}} \lesssim 0.2$, and (b) measurements of mean gradient between 0 and $\lambda$ are superficial if $\frac{\lambda}{x_{n}} \leqslant 0.5$. If $x_{f}$ represents the distance to the farthest point of the relief, then (c) a gradient measurement at $\lambda$ is not superficial if $\frac{\lambda}{x_{f}} \gtrsim \mathbf{0 . 5}$. These criteria are based upon the percentage variation of the topographic anomaly with depth, and they are independent of the height $(H)$ of the topographic relief. In this 
sense, the results (tables 1,2$)$ apply to depths $(\lambda)$ of 2 or 3 meters at distances $\left(x_{n}\right)$ of only $5-10$ meters from the toe or brink, even though , the height of the slope $(H)$ might be several kilometers. It has been shown that where $\frac{H}{x_{n}}$ is large, the Jeffreys approximation might not be valid. Therefore it is worth distinguishing between two cases: (1) $\frac{\lambda}{x_{n}}$ small, $\frac{H}{x_{n}}$ large and (2) $\frac{\lambda}{x_{n}}$ small, $\frac{H}{x_{n}}$ not large.

The validity of the superficial correction is assured by the first condition in each case. In the second case the Jeffreys approximation will also apply, whereas in the first it might not. Most geothermal observations in boreholes on the continents extend to a depth, $\lambda$, of the same order of magnitude as the topographic relief, $H$, even in rugged terrain. Hence, where the first condition is met, such observations will fall in case 2 and the Jeffreys approximation will usually apply to them. In geothermal observations at sea, however, the measurement depth $\lambda$ is normally only a few meters, and this may be less than the distance to nearby relief and very small relative to its height $(H)$ (case I). As an extreme example of case I, consider the gradient anomaly at the tip of a 2 -meter probe 20 meters from a cliff 2 kilometers high. Its value would be within 3 percent of the anomaly at the surface, that is, within 3 percent of $2.6 Q$ (table 1 ) or about $0.08 Q$. The error in the average gradient from neglecting this difference would be less than $1 \frac{1}{2}$ percent of $Q$. In this extreme example the error in applying the exact solution, valid at the surface, would be less by a factor of 2,000 than the error in applying the Jeffreys approximation with depth considered. As an illustration of case 2, steady-state topographic corrections throughout a 1,000-foot borehole can be computed from solutions valid at the surface if the (two-dimensional) topographic relief is more than a few thousand feet from the station. Under such circumstances the Jeffreys approximation and the exact solution would give comparable results unless the relief were very great. The topographic anomaly in the upper hundred feet of the hole could, of course, be considered superficial for relief extending to within a few hundred feet. For relief of one sign at any distance, the surface correction will provide an upper limit.

Although not directly amenable to treatment by present methods, cases other than the superficial ones $\left(\frac{\lambda}{x}\right.$ small $)$ should be mentioned in passing. It is seen from equation 106 and figure 21 that topographic elements for which $\frac{\lambda}{x} \cong 1$ have little or no direct effect on the gradient at depth $z$ irrespective of their height. Features for which $\frac{\lambda}{x}<1$ contribute to the topographic anomaly approximately as their height times the in- 
verse square of the measurement depth. If their height does not exceed the depth (the situation in all but the most rugged topography) their contribution is not large and can probably be estimated by the Jeffreys method. Large-scale topographic features that approach the station to within a fraction of the measurement depth cannot be treated generally with results for the superficial case although these results often provide useful limits. In such cases the doublet (or dipole) method of Jaeger and Sass (1963) might be used if a satisfactory representation of the surface can be obtained without an unwarranted effort. The depth variation (important to these cases) of the topographic anomaly can then be computed directly.

\section{OCEAN-BOTTOM GRADIENT MEASUREMENTS}

In oceanic geothermal studies the relief near a station is unknown because of limitations in present-day echo-sounding techniques. Even if the bottom were a horizontal plane, the echogram would generally be uncertain by a few meters at abyssal depths because of limits of instrumental precision, uncertainties in the sound velocity, and vertical movements of the ship on the open sea (Luskin and others, 1954; Krause and Menard, 1965; Heezen and others, 1959). If the sea bottom were irregular, uncertainties of elevation could be two orders of magnitude greater because the source of the echo is indeterminate within a circle of finite radius beneath the sonic source. For most instruments currently in use, the diameter of the circle is of the same order of magnitude as the water depth (kilometers) and reflections from positive features, generally not beneath the ship, tend to dominate the echogram (Krause, 1962). The net result is that the echogram generally yields a smoothed representation of the sea floor; if bold small-scale features exist, they are subdued or masked completely.

For example, it can be shown that the maximum relief, $d$, indicated by first arrivals on an echogram from parallel ridges separated by a distance, $\omega$, is given approximately by:

$$
d \cong \frac{\omega^{2}}{8 E}, \frac{\omega}{E} \lesssim 0.1
$$

where $E$ is the water depth. Thus features a few 10 's of meters wide in water a few kilometers deep would be masked; their indicated relief would be only a few centimeters. An echogram relief of 1 meter would be indicated in 4 kilometers of water by ridges 180 meters apart; the actual relief could be 10-100 times as great. Such a feature would subtend an angle of less than $3^{\circ}$ at the source, and probably would not be resolved even by high resolution techniques (Cohen, 1959).

We shall neglect topographic variations in the direction normal to the echo traverse, and investigate the magnitude of the topographic 
disturbance that could be caused by undetected relief. (This problem has been considered from a slightly different point of view in a recent paper by Birch, 1967.)

If the change in elevation of the bottom is uncertain by $\delta h$, then uncertainties in sea-bottom relief at distances beyond $n \delta h$ from the station could result in uncertainties in the topographic correction of roughly $2 \Delta q(n)$, where $\Delta q$ represents the anomaly due to a plane slope at $n=r$ or $n=s$ depending upon the sign of the error. (Such a slope may be viewed as the equivalent slope of very general configurations.) The factor 2 takes care of the case in which the error, $\delta h$, is of the same sign on both sides of the station; where $n$ is small the interaction should be considered (eq. 44, 46). As this is the worst case for the present discussion it will not be necessary to consider relief of both signs. From tables 1 and 2 it is seen that undetected relief at distances beyond $5 \delta h$ could cause uncertainties of 10 percent or so for positive relief, and 15 percent for negative relief. The uncertainty could be twice as great ( 20 or 30 percent) for $n=2$. Strictly speaking, these errors apply to the anomaly at the surface, $z=0$. However, it follows from equation 116 that if $n \delta h>2 \lambda$, the correction applicable to the mean gradient between $z=0$ and $z=\lambda$ must be close to the surface value (within 86 percent).

Very small amounts of undetected relief very close to the station can cause large anomalies in the surface gradient, but these decrease rapidly with depth. It has been shown that the change in the topographic anomaly over the depth $\lambda$ will be of the same general magnitude as the anomaly applicable at the surface if the relief is within $2 \lambda$ of the station and is all of one sign. Hence, if the temperature profile in $0<z<\lambda$ is linear, it can be reasonably assumed that significant relief does not occur on such a scale. If it is not, the mean gradient in $(0, \lambda)$ can contain an error from such relief equal to a substantial fraction of the observed change in gradient with depth.

If we rely upon curvature of the temperature profile as an indicator of undetected relief out to a distance $2 \lambda$, and apply the analysis of the preceding paragraph for relief beyond $n \delta h$, then all relief can be accounted for if:

$$
\lambda \cong \frac{n}{2} \delta h
$$

Thus, if the probe length, $\lambda$, is approximately equal to $\delta h$ (case $n=2$ ), the anomaly due to undetected (two-dimensional) relief could be roughly 20 percent plus a sizable fraction $\left(1 / 10^{-1 / 2}\right)$ of the observed change in heat flow over the length of the probe. For probes a few meters long the required $\delta h(\sim 2 \mathrm{~m})$ is probably approached by modern sounding techniques only where the sea bottom appears almost 
featureless in the echogram. For irregular sea bottoms where $\delta h$ can be several 10's of meters, equation 124 requires values of $n$ on the order of 0.1 for probes a few meters long. Under these conditions undetected relief could easily cause errors of 50 or 100 percent (tables 1,2 and eq 44 and 46). Such large errors from undetectable relief will often, but not always, be accompanied by a marked variation in the heat flow with depth, and curvature will flag the data as suspect. However, it is seen from tables 1 and 2 that if $\lambda$ is 2 meters and the relief is beyond 10 meters $(5 \lambda)$ with an angle of $15^{\mathrm{c}}$ and a height of a few 10's of meters, errors of 35-50 percent could occur with no detectable curvature in the temperature profile. It should be noted that these are the errors that could remain after the observable relief was corrected for. The corresponding three-dimensional results (with radial symmetry) might be greater by a factor of about 13.2. It is seen from equation 124 that one cannot be confident undetectable relief is causing errors less than 10 or 15 percent unless the probe length, $\lambda$, is at least $2 \frac{1}{2}$ times the uncertainty in local relief (case $n=5$ ) and the curvature of the temperature profile is negligible.

Although errors such as these may not be common, the possibility of their occurrence cannot be generally discarded until more is learned of the microrelief of the sea floor. These uncertainties are alleviated somewhat by bottom photography, and the situation will no doubt improve with the further development of high resolution sounding techniques (Cohen, 1959). The results emphasize the desirability of using longer thermal probes (such as the $10-\mathrm{m}$ device developed at Lamont Geol. Observatory) and for using several temperature sensors to detect curvature. If we discarded oceanic heat-flow data for which it could not be established that the curvature was less than 50 percent, we would probably be left with less than half of the world's heat-flow observations. At present, the best assurance against undetected topographic disturbances is probably the agreement of closely spaced measurements (Reitzel, 1963; Lachenbruch and Marshall, 1966).

It has been pointed out (Lachenbruch and Marshall, 1966; Langseth and others, 1966) that lateral heat flow from small-scale roughness can result in a systematic decrease in the mean regional gradient. Where such roughness has a wave length less than the probe length, $\lambda$, these effects can probably be identified as surficial ones if several temperature sensors are employed.

\section{TIME DEPENDENCE AND OTHER EFFECTS}

It has been pointed out by Birch (1950) that the finite times elapsed during and since the evolution of topography can have appreciable effects on the geothermal terrain correction. He presented 
a comprehensive theory, based on the Jeffreys approximation, to account for the disturbance to temperature at any depth beneath a general topographic surface in terms of rates of uplift and erosion. The two-dimensional case of Birch's theory was simplified by Clark (1957). The present paper concerns corrections to superficial gradient measurements. As we have seen, this is primarily a problem of accounting for close-in topography which can probably be considered to be in thermal equilibrium in most cases. However, an approximate theory of the transient effect will be considered to place the foregoing discussion in a time context. Where detailed transient corrections for distant topography seem warranted, the theory of Birch should be used.

The effects of any slope (positive or negative) are identified with the effects of its equivalent cliff. The cliff, at a distance $x$ from the station, is assumed to have evolved instantaneously at time $t=0$, and from that time onward its effect on the reference-plane temperature is taken as the value given by the Jeffreys approximation. The approach to equilibrium of the surface heat-flow anomaly at $x$ is described by (Lachenbruch, 1957, eq. 14 replacing $x$ with $-x$ ):

$$
\frac{\Delta q(x, t)}{\Delta q(x, \infty)}=\sqrt{\pi} \operatorname{ierfc} \frac{x}{2 \sqrt{\alpha t}}
$$

where $\alpha$ represents thermal diffusivity. Selected results from equation 125 are presented in table 6 for unconsolidated sediments $(\alpha=0.0025$ $\left.\mathrm{cm}^{2} \mathrm{sec}^{-1}\right)$ and rock $\left(\alpha=0.0125 \mathrm{~cm}^{2} \mathrm{sec}^{-1}\right)$. For topographic features whose height and distance is small relative to the sediment thickness, the value for sediment is probably more realistic. Larger, more distant features are probably represented better by the column headed "Rock".

TABLE 6.-Time, in years, for indicated percent approach of surface heat flow to equilibrium after generation of a cliff at distance $\mathrm{x}$

\begin{tabular}{|c|c|c|c|c|c|c|c|c|}
\hline \multirow{2}{*}{ Distance $x(\mathrm{~km})$} & \multicolumn{2}{|c|}{90 percent } & \multicolumn{2}{|c|}{50 percent } & \multicolumn{2}{|c|}{10 percent } & \multicolumn{2}{|c|}{$\Delta q$ for $1-\mathrm{km}$ cliff, $t=\infty$} \\
\hline & Rock & Sediment & Rock & Sediment & Rock & Sediment & $\begin{array}{c}\beta=+90^{\circ} \\
(x=r)\end{array}$ & $\begin{array}{c}\beta=-90^{\circ} \\
(x=s)\end{array}$ \\
\hline $\begin{array}{l}0.01 \\
0.1 \\
0.5 \\
1.0 \\
50 \\
10\end{array}$ & $\begin{array}{l}1.8 \times 10^{2} \\
1.8 \times 10^{4} \\
4.5 \times 10^{5} \\
1.8 \times 10^{6} \\
4.5 \times 10^{7} \\
1.8 \times 10^{8} \\
4.5 \times 10^{9}\end{array}$ & $\begin{array}{l}9.0 \times 10^{2} \\
9.0 \times 10^{4} \\
2.3 \times 10^{6} \\
9.0 \times 10^{6} \\
2.3 \times 10^{8} \\
9.1 \times 10^{8} \\
2.3 \times 10^{10}\end{array}$ & $\begin{array}{l}\text { 5. } 2 \\
\text { 5. } 2 \times 10^{2} \\
\text { 1. } 3 \times 10^{4} \\
\text { 5. } 2 \times 10^{4} \\
\text { 1. } 3 \times 10^{6} \\
\text { 5. } 2 \times 10^{6} \\
\text { 1. } 3 \times 10^{8}\end{array}$ & $\begin{array}{l}26 \\
2.6 \times 10^{3} \\
6.5 \times 10^{4} \\
2.6 \times 10^{5} \\
6.5 \times 10^{6} \\
2.6 \times 10^{7} \\
6.5 \times 10^{8}\end{array}$ & $\begin{array}{l}0.7 \\
68 \\
1.7 \times 10^{3} \\
6.8 \times 10^{3} \\
1.7 \times 10^{5} \\
6.8 \times 10^{5} \\
1.7 \times 10^{7}\end{array}$ & $\begin{array}{l}\text { 0. } 3 \\
\text { 3. } 4 \times 10^{2} \\
8.5 \times 10^{3} \\
\text { 3. } 4 \times 10^{4} \\
\text { 8. } 5 \times 10^{5} \\
\text { 3. } 4 \times 10^{6} \\
\text { 8. } 5 \times 10^{7}\end{array}$ & $\begin{array}{l}+2.9 \\
+.86 \\
+.32 \\
+.20 \\
+.05 \\
+.03 \\
+.006\end{array}$ & $\begin{array}{l}-0.99 \\
-.92 \\
-.64 \\
-.41 \\
=.08 \\
=.04 \\
-.006\end{array}$ \\
\hline
\end{tabular}

From the second line of table 6 it is seen that an open pit or mine dump made in this century would not affect the surface heat flow in a borehole only 100 meters away. By inequality 111 , the result applies to gradients throughout the borehole. Fairly uniform relief, ap- 
proaching to within about 1 kilometer of the station, can be described by the equilibrium theory if it has not changed much since early Pliocene time if the sediments are thick, or early Pleistocene if they are thin. The latter alternative would apply, for example, to the walls of an oceanic trench for stations on the floor. A substantial fraction of the effect of slopes forming 10 million years ago would be felt at stations 5 or 10 kilometers away. It is seen from the last column of table 6 that effects of such slopes would generally be small.

The results of the previous section are based on the assumption that the earth's thermal properties are uniform. Over much of the ocean basins a layer of unconsolidated sediments about 1-kilometer thick overlies more consolidated material of contrasting properties. Where the topographic features are composed of unconsolidated sediments and their distance from the station is not large relative to the thickness of the sedimentary layer, the homogeneous model probably applies reasonably well. Where the sedimentary layer is thin relative to the relief, the homogeneous model should again be applicable. Lateral inhomogeneities in thermal properties can cause appreciable heat-flow anomalies, and they must be considered separately (Von Herzen and Uyeda, 1963; Lachenbruch and Marshall, 1966). One such problem is considered briefly below.

For convenience it has been assumed that the topographic surface is isothermal. If the temperature of the surface decreases linearly with elevation with gradient, $G^{\prime}$, then the topographic anomaly would be given by:

$$
\frac{G-G^{\prime}}{G} \Delta g
$$

For terrain above sea level, approximations to $G^{\prime}$ are found to range from about $3^{\circ}$ to $9^{\circ} \mathrm{C}$ per kilometer, and this value is often $10-50$ percent of $G$. Such values of $G^{\prime}$ result in substantial reductions of the topographic anomaly. At abyssal depths in the ocean we normally have $G^{\prime} / G \sim 10^{\boldsymbol{r}^{3}}$, as $G^{\prime}$ is of the order of the adiabatic gradient in sea water, and the assumption that the surface is isothermal is realistic.

\section{BURIED BEDROCK SLOPE}

Equation 126 suggests an additional application of the results for plane slopes. Suppose a bedrock surface dips under sedimentary material of conductivity $K_{1}$ and there is no topographic expression at the surface, as illustrated in figure 23 . If the conductivity of the rock is $K$, then the gradient $G_{1}$ in the sediment at points distant from the slope is:

$$
G_{1}=\frac{K}{K_{1}} G
$$




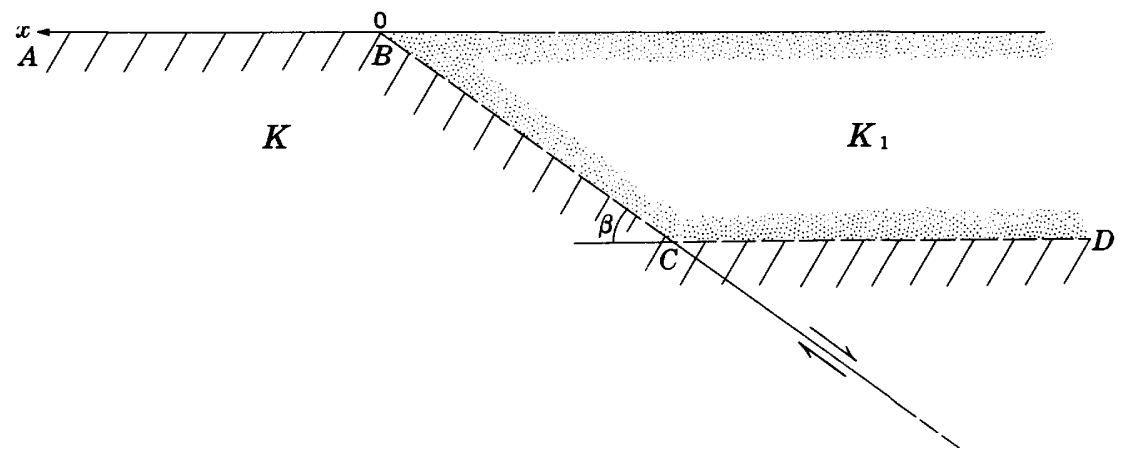

Figure 23.-Model of the downfaulted bedrock pediment.

As an approximation, we assume that the gradient $G_{1}$ obtains throughout the sediment above the bedrock surface. Replacing $G^{\prime}$ by $G_{1}$ in equation 126 yields:

$$
\Delta q_{b}\left(x_{0}, \beta\right) \cong\left(1-\frac{K}{K_{1}}\right) \Delta q\left(x_{0}, \beta\right),
$$

where $\Delta q_{b}$ is the heat-flow anomaly caused by the buried bedrock topography at points on the surface behind the brink (fig. 23, $A B$ ) and at the buried interface (fig. $23, B C D$ ).

If the conductivity of the bedrock is approximately twice that of the sediment (a common situation), the lower curves in figure 3 give the negative of the anomaly along $A B$ (fig. 23), and the upper curves of figure 3 give the negative of the anomaly along the interface $C D$ (fig. 23).

This model describes a common situation in the Basin and Range province of the Western United States, where bedrock pediment surfaces are downfaulted on the basin side and the depression is subsequently filled with alluvium. The results are useful in the interpretation of geothermal data from boreholes in such areas.

\section{SUMMARY}

The effect of topographic relief on heat flux through the surface can be determined exactly for a semi-infinite medium bounded by a plane slope (two horizontal half planes joined by an inclined plane segment) where the vertical heat flow is uniform at great depth, and the surface temperature varies linearly with elevation. Analytical results are given for vertical flux across both the horizontal and sloping portions of the surface for slope angles of $\frac{\pi}{n}, n$ an even integer greater than or equal to 2 (eq 12 and 14) and for $n=3$ (eq 13). They form the basis for graphs and charts that yield results for all slope 
angles between 0 and $\frac{\pi}{2}$. The solution is a generalization of the work of Castoldi (1952) who considered the vertical cliff, $n=2$. These results can be used in various ways to estimate the effect of topographic relief on heat flow across the earth's surface. They may be most useful in oceanic geothermal studies where the gradient measurement is of ten superficial, and the temperature condition on the solid surface is generally satisfied rather well. The simplicity of the geometric model is both a weakness and a strength in such applications. It is a weakness because there are no such slopes on the earth's surface. It is a strength because the two parameters, slope angle $(\beta)$ and slope height $(H)$, are so easily visualized and represented graphically that models which bracket or approximate real topography can be identified quickly.

Certain results of interest in geothermal studies generally follow directly from the exact solutions. If a plane slope can be drawn in such a way that it is tangent to the real topographic surface at the station and not below it elsewhere, then the exact solution for that plane slope gives an upper limit to the topographic anomaly at the station. Similarly, a plane slope tangent to the real surface at the station, and not above it elsewhere, yields a lower limit to the anomaly. The limits imposed by such geometric bracketing can be read directly from graphs (figs. 3,$4 ;$ pl. 1). The method provides rapid means of establishing whether or not more elaborate topographic corrections are needed. Thus, it follows from the case $\beta=90^{\circ}$ (tables 1 and 2) that any two-dimensional topographic feature whose height is less than 10 percent of its distance from the station has little effect on the gradient at the surface. (By inequality 111 its effect is negligible at all depths.) The exact results can sometimes be applied directly to eliminate topographic effects as an explanation of anomalous heat-flow results. For example, a heat flow four times the regional average, such as some reported from oceanic rises, could be caused by a vertical cliff 1 kilometer high only if the station were less than 7 meters from its foot (table 1). Such a topographic setting could hardly go undetected. The same anomaly at the surface could, of course, be caused by a 100-meter cliff 70 centimeters from the station or a 10-meter cliff 7 centimeters from the station, but it would not persist to depths on the order of 1 meter.

From the exact solution for flux through a plane slope it is possible to derive expressions for upper and lower limits to the topographic anomaly on the flat surface between two plane slopes of any height or slope angle. There are three cases: the plane valley, the plane ridge, and the plane bench. In the plane valley (inequality 44 ) the station lies on the horizontal surface between two plane slopes rising 
above the station (such slopes are called positive). In the plane ridge, the station lies on the horizontal surface between two negative plane slopes (inequality 46). The plane bench represents a station on the horizontal surface between plane slopes of opposite sign (inequality $49 \mathrm{a}, \mathrm{b})$. For both the valley and the ridge, a lower limit to the topographic anomaly is obtained by adding the individual anomalies caused by each slope, that is, by neglecting the interaction. Both the upper and lower limit for the bench contain interaction terms. As the slopes become smaller or farther apart, so that the effect of each on the other decreases, the bracketing intervals diminish, and the sum of the independent anomalies from each slope becomes a good approximation in all three cases. The parameters in the inequalities 44,46 , and $49 \mathrm{a}, \mathrm{b}$ can be obtained directly from plate 1 or tables 1 and 2. By geometric bracketing the results can be used to obtain limits to the topographic anomaly for stations on horizontal surfaces in real valleys, ridges, or benches. Thus, the topographic anomaly in the center of an oceanic trench 2 kilometers deep and 2 kilometers wide at the bottom and that has irregular walls sloping generally between $5^{\circ}$ and $9^{\circ}$ (Fisher and Hess, 1963) is between 18 and 29 percent (table 1 and inequality 44).

Although the immediate results of the exact solution for a plane slope can be useful, their application is limited by (1) the fact that geometric bracketing often yields bracketing intervals too wide to be helpful, (2) most of the generalizations apply to stations on geometrically horizontal surfaces, rare in nature, and (3) the solution gives no information about the variations of the topographic anomaly with depth.

These limitations can be relaxed with the aid of the approximate solution of Jeffreys (1938) for the surface heat-flow anomaly $\Delta q^{\prime}\left(x_{0}\right)$, at the station $x_{0}$ caused by two-dimensional topographic relief, $h(x)$.

$$
\Delta q^{\prime}\left(x_{0}\right)=\frac{1}{\pi} \int_{-\infty}^{+\infty} \frac{h(x)}{\left(x-x_{0}\right)^{2}} d x .
$$

The solution is based on the widely used simplification, in which the irregular topographic surface is replaced by a horizontal plane through the station. On this reference plane the temperature is assumed to vary as $G h(x)$, where $G$ represents the regional thermal gradient and $h\left(x_{0}\right)$ is taken as zero. The advantage of the approximation lies in the fact that it is linear in the sense that the effects of individual topographic elements can be superimposed to obtain the total effect of the relief. The result is only an approximation because it fails to account for the effects on vertical gradient of lateral heat loss through 
the sloping surfaces. The expression (128) can be made exact with the following modification:

$$
\Delta q\left(x_{0}\right)=\frac{1}{\pi} \int_{-\infty}^{+\infty} \frac{h(x)[1+e(x)]}{\left(x-x_{0}\right)^{2}} d x,
$$

where $e(x)$ is an unknown function whose value at each point depends upon $h(x)$ at all points. Physically it represents the mean gradient anomaly in $h(x)$ at $x$; its value is generally greater than -1 .

At any point $x_{0}$ on the horizontal portions of a plane slope, there is a family of plane slopes of equal height that will yield the same linear approximation to the topographic anomaly, $\Delta q^{\prime}\left(x_{0}\right)$; the steeper ones will terminate farther from the station, the flatter ones closer to it. We refer to such slopes as equivalent at $x_{0}$. Each member of a family of equivalent plane slopes will yield a different value for the exact anomaly, $\Delta q\left(x_{0}\right)$. By equations 128 and 129 the difference will correspond to differences in the quantity:

$$
\frac{1}{\pi} \int_{-\infty}^{+\infty} \frac{h e}{\left(x-x_{0}\right)^{2}} d x
$$

However, $e$ is a strongly decreasing function of distance from the toe. Therefore, for positive $h$, the steeper equivalent slopes should generally yield smaller anomalies because, for them, $h$ is smaller and farther from the station where $e$ is large, and it is larger and closer to the station where $e$ is small. A similar argument applies for negative $h$. It has been demonstrated numerically (fig. 14-16 and tables 4, 5) that in any family of equivalent plane slopes the topographic anomaly does, indeed, decrease with increasing slope angle except for points very close to the brink of steep slopes. There the heat flow is nearly zero and small effects of higher order, relating to differences in the $e$ 's can dominate. Such departures are small and generally unimportant.

This relation suggests a more refined method of bracketing. To generalize the plane slope, and still restrict the discussion to related slopelike forms, we define a slope form as two horizontal half planes joined by a general (two-dimensional) surface whose highest point is the intersection with the upper half-plane (the brink), and whose lowest point is the intersection with the lower half-plane (the toe). At any point, $x_{0}$, on the horizontal portion of a slope form (excluding the toe and brink) there is a family of equivalent plane slopes. In this family there are some members, $h^{*}(x)$, so steep that they cross the given slope at only one point, falling below it near the toe and above it near the brink. There are other members, $\hat{h}(x)$, so flat that they cross it only once and lie above it near the toe and below it near the 
brink. It is shown (inequality 71) that we can generally expect the exact topographic anomaly from a slope form to be greater than that caused by the steeper equivalent plane slopes $\left(h^{*}(x)\right)$ and less than that caused by the flatter equivalent plane slopes $(\hat{h}(x))$. The result can be visualized intuitively by noting that the difference between each bracketing plane slope and the given slope is one positive and one negative topographic region. If the gradients in each were uniform, their effects on reference-plane temperature would cancel at $x_{0}$ because of equivalence. However, because the gradient is generally greater near the toe, the difference takes the sign of the region there; positive for $\hat{h}$ and negative for $h^{*}$. The justification of the bracketing inequality 71 involves neglecting certain higher order effects associated with differences in the $e^{\prime}$ s. The effects are probably unimportant in geothermal applications; but the matter may deserve further study.

Bracketing the effects of a general slope form with equivalent plane slopes can be accomplished quickly in two or three steps by determining $\Delta q^{\prime}\left(x_{0}\right)$ from plate 2 and then using plate 1 or figures 15 or 16. A very good approximation to the effects of a general slope form can usually be obtained by selecting an equivalent plane slope to approximate rather than to bracket.

A positive slope form for which it is possible to draw a flatter equivalent plane slope, $\hat{h}$, through the toe is called "concave at the toe"; a negative one for which $\hat{h}$ can be drawn through the brink is called "convex at the brink." Such slopes can be replaced by their equivalent cliffs with very small (negative) error as long as their height does not exceed their distance from the station.

Consideration of equivalent slopes yields some conditions for validity of the Jeffreys approximation at the surface. For example, the Jeffreys method can be used with negligible error for positive slope forms, concave at the toe, whose height does not exceed 20 times the distance from the station and whose maximum slope angle is less than $30^{\circ}$. This rule includes cases with positive anomalies ranging up to 65 percent. The approximation applies also to negative slope forms inclined less than $45^{\circ}$ if they are convex at the brink and their height is less than three times their distance from the station. Virtually any slope of one sign can be represented by the Jeffreys method as long as it does not produce an anomaly exceeding 10 or 15 percent.

Although the slope form is rather general, its application to real topography is limited by the fact that the sloping parts are of finite width and the station must lie on a horizontal surface, though it may be arbitrarily close to the sloping portions. Virtually all heatflow stations lie on surfaces that depart from a horizontal plane, but the curvature and slope can often be considered to be very small, 
especially for stations on the ocean floor. If the real surface, $h(x)$, is gently sloping and smooth over an interval of width $l$, centered at the station, then the error arising from neglecting its slope and curvature is equation 90 :

$$
\frac{l}{\pi}\left[q\left(x_{0}\right) \frac{d^{2} h}{d x^{2}}+2 \frac{d h}{d x} \frac{d q}{d x}\right] .
$$

The result is obtained by flattening the region $l$ by replacing the real slope by a horizontal surface through the station $\left(x_{0}\right)$, and adjusting the remainder of the relief upward or downward to eliminate discontinuities at $x=x_{0} \pm l / 2$. The result accounts for the reaction of the local relief to the distant relief which can be substantial, but it neglects the higher order effect of the reaction of the distant relief to the subdued local relief. It is seen that the anomaly caused by gentle local relief is independent of the absolute heat flow if the curvature is negligible, and independent of the lateral heat-flow gradient if the slope is negligible. Generally, flattening errors will not exceed a few percent if the slope at the station is less than $4^{\circ}$ or $5^{\circ}$ and the change in slope angle over the distance $l$ does not exceed $2^{\circ}$ or $3^{\circ}$ (see eq 91). After flattening, the station will often lie on the horizontal surface between two slope forms (the valley, ridge, or bench), one of which can commonly be neglected.

Some knowledge of the variation of the topographic anomaly with depth is extremely important in the application of the foregoing results. Fortunately it is possible to modify the Jeffreys approximation for the topographic anomaly as a function of depth, just as equation 128 was modified to obtain equation 129, that is, by introducing $e(x)$ into the integrand to make it an exact expression. Thus, equation 106 describes exactly the topographic anomaly due to any two-dimensional topographic surface as a function of depth and horizontal position. Even though $e(x)$ is unknown, many useful results can be obtained.

If the efiective topography is all of one sign (for example, if the station is at the edge of an abyssal plane or shelf or in the trough or ridge of ripple marks), the topographic anomaly at any station, $x_{0}$, attains its greatest magnitude at the surface $z=0$ (inequality 111).

The result applies also to the transient case if the direction of vertical movement was of the same sign as the relief during topographic evolution. The result does not apply to the error in the Jeffreys approximation, and hence the error does not, in general, attain its greatest magnitude at the surface.

Limits to the percentage variation of the topographic anomaly with depth can be expressed in terms of horizontal distance to the edge of a (two-dimensional) topographic feature, without reference to its 
height. The gradient anomaly at depth $\lambda$ is approximated well by the value applicable at the surface if the relief is of one sign and is farther from the station than $5 \lambda$. The mean gradient anomaly in the depth interval $(0, \lambda)$ can be approximated by the surface value for relief of one sign approaching to within $2 \lambda$. (The same statements apply to relief of both signs in the sense that the error is generally a small percentage of the sum of the magnitudes of the individual contributions of the positive and negative portions.) Under these conditions the gradient anomaly is said to be superficial and the exact results for plane slopes can be applied to them. (For example, tables 1 and 2 apply to depths of 2 or 3 meters at distances of only 5 or 10 meters from the toe or brink, even though the height of the slope may be measured in kilometers.) Where the height of the relief is large relative to its distance from the station, the Jeffreys approximation cannot be applied to superficial gradients with confidence, whereas the present method can. Whether or not the Jeffreys approximation applies, the present method can yield rapid estimates for two-dimensional superficial cases. The effect on the gradient at depth $\lambda$, of relief lying entirely within a horizontal distance $2 \lambda$, is generally an order of magnitude less than its effect at the surface. Unless the height is greater than $\lambda$, the Jeffreys approximation will probably apply. Large-scale features extending to within $2 \lambda$ of the station cannot be treated directly by the present methods although useful limits can sometimes be obtained.

Oceanic geothermal measurements can contain errors greater than 10 percent from undetected relief unless the probe is at least $2 \frac{1}{2}$ times as long as the uncertainty in local elevations and the curvature of the temperature profile is negligible. If the probe length is equal to the uncertainty in local relief, an individual measurement can contain errors on the order of 20 percent plus a substantial fraction of the change in gradient over the length of the probe. Changes in local elevations determined over an irregular sea bottom by modern sounding techniques can be uncertain by 10 's or even 100 's of meters. This undetected relief can cause gradient errors of 50-100 percent in measurements to depths of a few meters. Such measurements will often, but not always, be flagged as suspect by marked variation in the heat flow with depth. The occurrence of sea-bottom relief on a scale important for geothermal measurements is largely unknown. At present the best assurance against undetected topographic anomalies is the agreement of closely spaced observations.

The major problem in estimating the topographic disturbance to superficial gradients is to account for the close-in features. Inasmuch as they have short time constants, the steady-state theory is probably applicable in most cases (table 6). The small effects of topographic 
evolution of distant features can be treated adequately with existing methods (Birch, 1950).

\section{REFERENCES CITED}

Birch, Francis, 1950, Flow of heat in the Front Range, Colorado: Geol. Soc. America Bull., v. 61, no. 6, p. 567-630.

1967, Low values of oceanic heat flow: Jour. Geophys. Research, v. 72, no. 8 , p. 2261-2262.

Bullard, E. C., 1938, The disturbance of the temperature gradient in the earth's crust by inequalities of height: Royal Astron. Soc. Monthly Notices, Geophys. Supp., v. 4, May, p. 360-362.

Bullard, E. C., Maxwell, A. E., and Revelle, R. R. D., 1956, Heat flow through the deep sea floor: Advances in Geophysics, v. 3, p. 153-181.

Castoldi, Luigi, 1952, Sulla distribuzione della temperatura negli strati superiori della crosta terrestre [On the temperature distribution in the upper layers of the earth's crust]: Geofisica Pura e Appl., v. 23, p. 27-35.

Clark, S. P., Jr., 1957, Heat flow at Grass Valley, California: Am. Geophys. Union Trans., v. 38, no. 2, p. 239-244.

Cohen, P. M., 1959, Direction echo sounding on hydrographic surveys: Internat. Hydrog. Rev., v. 36, p. 29-42.

Fisher, R. L., and Hess, H. H., 1963, Trenches, in Hill, M. N., ed., The earth beneath the sea, Volume 3 of The sea: New York, John Wiley \& Sons, p. 411436.

Gröbner, Wolfgang, and Hofreiter, Nikolaus, ed., 1949, Unbestimmte Integrale, Pt. 1 of Integraltafel: Vienna and Innsbruck, Springer-Verlag, 166 p.

Heezen, B. C., Tharp, Marie, and Ewing, Maurice, 1959, The North Atlantictext to accompany the physiographic diagram of the North Atlantic, [Part] 1 of The floors of the oceans: Geol. Soc. America Spec. Paper 65, $122 \mathrm{p}$.

Jaeger, J. C., 1965, Application of the theory of heat conduction to geothermal measurements, in Lee, W. H. K., ed., Terrestrial heat flow: Am. Geophys. Union Geophys. Mon. Ser., no. 8, p. 7-23.

Jaeger, J. C., and Sass, J. H., 1963, Lee's topographic correction in heat flow and the geothermal flux in Tasmania: Geofisica Pura e Appl., v. 54, p. 53-63.

Jeffreys, Harold, 1938, The disturbance of the temperature gradient in the earth's crust by inequalities of height: Royal Astron. Soc. Monthly Notices, Geophys. Supp., v. 4, January, p. 309-312.

Kober, H., 1952, Dictionary of conformal representations: New York, Dover Pub., Inc., $208 \mathrm{p}$.

Krause, D. C., 1962, Interpretation of echo sounding profiles: Internat. Hydrog. Rev., v. 39, p. 65-123.

Krause, D. C., and Menard, H. W., 1965, Depth distribution and bathymetric classification of some sea-floor profiles: Marine Geology, v. 3, p. 169-193.

Lachenbruch, A. H., 1957, Thermal effects of the ocean on permafrost: Geol. Soc. America Bull., v. 68, no. 11, p. 1151-1529.

Lachenbruch, A. H., and Marshall, B. V., 1966, Heat flow through the Arctic Ocean floor-The Canada Basin-Alpha Rise boundary: Jour. Geophys. Research, v. 7, no. 4, p. 1223-1248.

Langseth, M. G., Jr., Le Pichon, Xavier, and Ewing, Maurice, 1966, Heat flow through the Atlantic Ocean floor and convection currents, [Part] 5 of Crustal structure of the mid-ocean ridges: Jour. Geophys. Research, v. 71, no. 22, p. 5321-5355. 
Lee, W. H. K., and Uyeda, Seiya, 1965, Review of heat flow data, in Lee, W. H. K., ed., Terrestrial heat flow: Am. Geophys. Union Geophys. Mon. Ser., no. 8, p. 87-190.

Lees, C. H., 1910, On the shapes of the isogeotherms under mountain ranges in radioactive districts: Royal Soc. London Proc., v. 83 (A 563), p. 339-346.

Luskin, Bernard, Heezen, B. C., Ewing, Maurice, and Landisman, Mark, 1954, Precision measurement of ocean depth: Deep-Sea Research, v. 1, no. 3, p. 131-140.

Reitzel, John, 1963, A region of uniform heat flow in the North Atlantic: Jour. Geophys. Research, v. 68, no. 18, p. 5191-5196.

Von Herzen, R. P., and Uyeda, Seiya, 1963, Heat flow through the eastern Pacific Ocean floor: Jour. Geophys. Research, v. 68, no. 14, p. 4219-4250. 


\section{Experimental and}

\section{Theoretical Geophysics}

\section{4-68}
GE O L O G I A L
S U R V E Y
B U L L E T I N
1203

This volume was published as separate chapters $A-E$

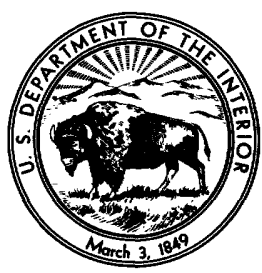


UNITED STATES DEPARTMENT OF THE INTERIOR

WALTER J. HICKEL, Secretary

GEOLOGIGAL SURVEY

William T. Pecora, Director

Library of Congress catalog-card No. GS 68-364 


\section{CONTENTS}

[Letters designate the separately published chapters]

(A) Measurement of the remanent magnetization of igneous rocks, by Richard R. Doell and Allan Cox.

(B) Properties of thermistors used in geothermal investigations, by Eugene C. Robertson, Rudolph Raspet, Joel H. Swartz, and Major E. Lillard.

(C) Preparation of thermistor cables used in geothermal investigations, by Rudolph Raspet, Joel H. Swartz, Major E. Lillard, and Eugene C. Robertson.

(D) Method-dependent values of bulk, grain, and pore volume as related to observed porosity, by G. Edward Manger.

(E) The effect of two-dimensional topography on superficial thermal gradients, by Arthur H. Lachenbruch. 
